* 

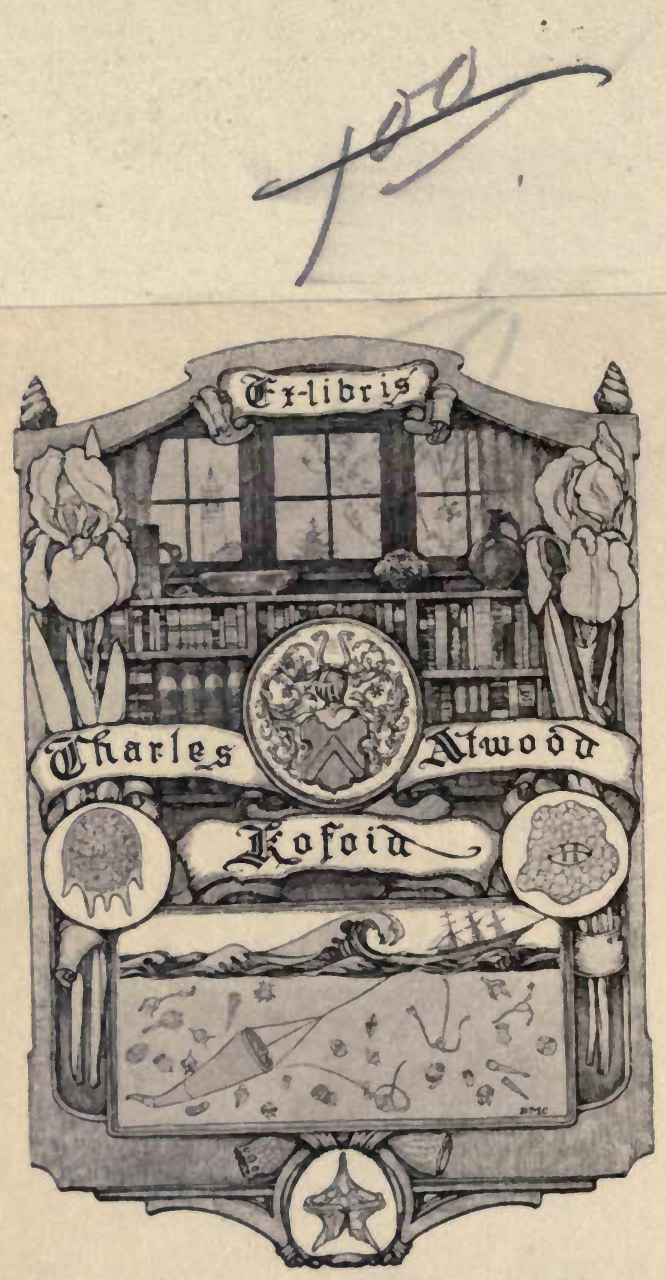


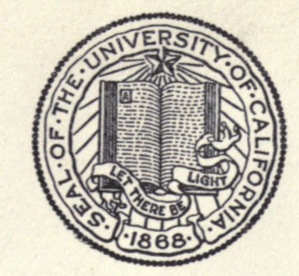

THE LIBRARY

OF

THE UNIVERSITY OF CALIFORNIA

PRESENTED BY

PROF. CHARLES A. KOFOID AND

MRS. PRUDENCE W. KOFOID 






\section{THE SCIENCE SERIES}

I. The Study of Man. By A. C. HADDon. Illustrated. $8^{\circ}$

2. The Groundwork of Science. By St. George MIVART.

3. Rivers of North America. By Israel C. Russell. Illustrated.

4. Earth Sculpture; or, The Origin of Land Forms. By James Geikie. Illustrated.

5. Volcanoes; Their Structure and Significance. By T. G. BonNey. Illustrated.

6. Bacteria. By George Newman. Illustrated.

7. A Book of Whales. By F. E. BEDDARD. Illustrated.

8. Comparative Physiology of the Brain, etc. By JACQUES LOEB. Illustrated.

9. The Stars. By Simon Newcomв. Illustrated.

ro. The Basis of Social Relations. By DANIEL G. Brinton.

II. Experiments on Animals. By Stephen Paget.

12. Infection and Immunity. By George M. Sternberg.

For list of works in preparation see end of this volume. 


\section{Tbe ฐcience ฐeries}

EDITED BY

Professor Э. Incikeen CatteII, ID.F., Pb.ஐ.

AND

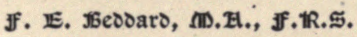




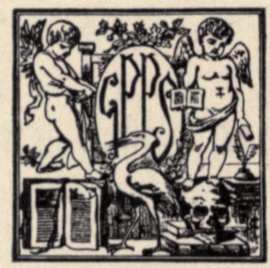




\title{
Infection and Immunity
}

With Special Reference to

\section{The Prevention of Infectious Diseases}

\author{
By \\ George M. Sternberg, M.D., LL.D. \\ Surgeon-General U. S. Army (Retired) \\ Ex-President of the American Medical Association, and of the American Public \\ Health Association; Honorary Member of the Epidemiological Society \\ of London, of the Sociéte Française d'Hygiène, of the \\ Royal Academy of Medicine of Rome, etc.
}

G. P. Putnam's Sons

New York and London

Tbe $\mathbb{k}$ nickerbocker $\mathbb{p r e s g}$

I 903 
COPYRIGHT, I9O3

BY

G. P. PUTNAM'S SONS

Published, October, 1903

The Iknickerbocker Dress, Thew york 


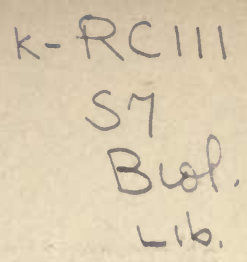

\section{PREFACE}

$\mathrm{N}$ this volume the writer has attempted to state the main facts, so far as they have been established, with reference to infection and immunity, with the practical object in view of indicating the measures necessary for the prevention of infectious diseases. As the work is intended for non-medical readers, I have avoided technical terms as far as practicable, and when it has been necessary to use these have endeavoured to explain them. I have thought it best not to enter upon a discussion of the theories of immunity, or to attempt to give an account of the results of recent investigations with reference to the " antitoxins," "agglutinins," " precipitins," " bacteriolysins," etc. This line of investigation has, during the past few years, been so prolific in surprising results, and so many new technical terms have become necessary for the designation of the newly discovered bodies of this class and for the understanding of Ehrlich's "side-chain theory," which attempts to explain their mode of action, that this subject does not, 
at present, seem suitable for popular treatment. In my opinion, a knowledge of the well established facts in this field of investigation should constitute an essential part of a liberal education; and the diffusion of such knowledge cannot fail to promote the sanitary interests of the people.

The general statement may be made that all infectious diseases are preventable diseases, and at the present time it is possible to indicate the necessary measures of prevention for nearly all of these diseases. That they continue to prevail, and to claim hundreds of thousands of victims annually, is largely due to the fact that the public, generally, has not yet been educated upon these subjects.

It would seem that so important a matter should receive special attention in our high schools and colleges, and the writer hopes that this volume may, to some extent at least, serve as a text-book, suitable for the use of students, and as a manual of ready reference for those who are responsible for the sanitary welfare of the inmates of homes, schools, public institutions, etc. In Part Second the most important infectious diseases are considered in special chapters, and an attempt has been made to indicate the manner in which each one is propagated, its importance as a factor in our mortality statistics, and the best methods of restricting its extension. I have been strongly 
tempted to write an additional chapter upon venereal diseases, but have refrained from treating this unsavoury topic. I may, however, be permitted to quote here from the concluding paragraph of my "Lomb Prize Essay," upon Disinfection and Personal Prophylaxis against Infectious Diseases, published by the American Public Health Association in 1886 :

"This clapter might be greatly extended, but, having passed in review the principal measures of individual prophylaxis against those infectious diseases which are most fatal, we shall not dwell upon precautions to be taken in other contagious diseases. These precautions will not differ from those already recommended in the cases of smallpox and scarlet fever. So, too, in regard to the infectious skin diseases. These are communicated by personal contact, and rarely occur except among those who neglect personal cleanliness as well as other sanitary laws. Soap and water will generally suffice for individual prophylaxis. By avoiding filthy persons as well as filthy places, the danger of contracting these and certain other unmentionable infectious diseases will be reduced to a minimum."

$$
\text { G. M. S. }
$$

2144 California Avenue,

WASHINGTON, Fune 10, 1903. 



\title{
CONTENTS
}

\author{
PART FIRST
}

CHAPTER I

General Remarks upon Infection

CHAPTER II

Disease Germs

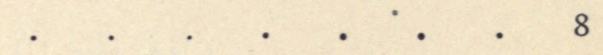

CHAPTER III

Channels of Infection .

CHAPTER IV

Susceptibility to Infection . • . • • • 23

CHAPTER V

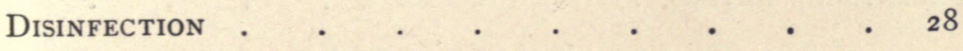

CHAPTER VI

Tests of Disinfection • . • • • • • $3^{2}$

CHAPTER VII

Disinfection by Heat • . • • • • • 43

CHAPTER VIII

Sunlight as a Disinfectant . . • • 48

CHAPTER IX

Disinfection by Gases • • • • • • • $5^{\mathbf{I}}$

CHAPTER $\mathrm{X}$

Various Chemical Disinfectants . $\quad$. $\quad 57$ 
Natural Immunity .

CHAPTER XII

ACQuired Immunity

CHAPTER XIII

Antitoxins

\section{PART SECOND}

CHAPTER I

Bubonic Plague

ÇHAPTER II

Asiatic Cholera

\section{CHAPTER III}

Typhoid Fever

chapter iv

Dysentery, Cholera Infantum, etc. .

CHAPTER V

Relapsing Fever .

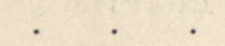

Typhus Fever.

CHAPTER VII

Tuberculosis .

\section{CHAPTER VIII}

LEPROSY .

CHAPTER IX

Diphtheria

\section{CHAPTER $\mathrm{X}$}

INFLUENZA 
CONTENTS

CHAPTER XI

PAGE

Pneumonia

\section{CHAPTER XII}

Whooping-Cough .

CHAPTER XIII

SmallpoX

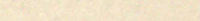

CHAPTER XIV

Scarlet Fever

\section{CHAPTER XV}

Measles

CHAPTER XVI

Malarial Fevers .

CHAPTER XVII

Yellow Fever

\section{CHAPTER XVIII}

WOUND INFECTIONS .

CHAPTER XIX

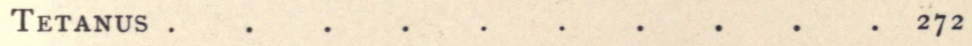
CHAPTER XX
HYDROPHOBIA • • • • • • • • 279 INDEX $\cdot \quad \cdot \quad \cdot \quad \cdot \quad \cdot \quad \cdot 289$




\section{ILLUSTRATIONS}

FIGURE

I.-Bacillus of Bubonic Plague . • . . IoI

2.-Spirillum of Asiatic Cholera ("Comma Bacil-

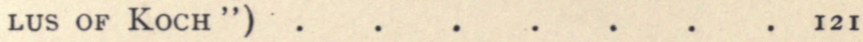

3.-Bacillus of Trphoid Fever . . . . . 136

4.-Spirillum of Relapsing Fever, as Seen in the

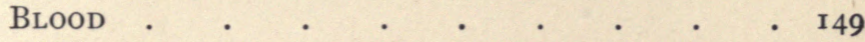

5.-Bacillus of Tuberculosis, as Seen in the Sputum of a Patient Having Pulmonary Consumption . i6o

6.-Bacillus of Leprosy, as Seen in a Thin Section OF a Leprous Nodule . . . . . . . I82

7.-Bacillus of Diphtheria $\quad . \quad$. $\quad . \quad$ I93

8.-Bacillus of Influenza . . . . . 200

9.-Micrococcus of Pneumonia . . . 206

io.-Micrococcus of Pus-formation . • • 266

I I.-Micrococcus of ERysipelas, EtC. • • . $\quad 267$

12.-Bacillus of Tetanus • • • • • 273 



\section{PART FIRST}

INFECTION, DISINFECTION, AND IMMUNITY 


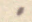




\section{CHAPTER I}

GENERAL REMARKS UPON INFECTION

$M^{\mathrm{UCH}}$ confusion exists in the popular use of the term "infection." By many it is supposed to be synonymous with contagion. But this is not in accordance with established usage among well-informed physicians. It is a far more comprehensive term ; for, while all contagious diseases are infectious, not all infectious diseases are contagious. A contagious disease is one which may be transmitted by personal contact, as, for example, smallpox, measles, scarlet fever. These diseases may also be communicated, indirectly, through the medium of objects which have been in contact with infected individuals, such as clothing, bedding, etc. ("fomites"). While the contagious diseases mentioned and others which are generally recognised as liable to be contracted by contact with the sick are also infectious, there are numerous diseases which are infectious but not contagious. As examples of this class we may mention the malarial 
fevers, yellow fever, and trichinosis. Still others, such as cholera, typhoid fever, and dysentery are only transmitted by personal contact under very exceptional circumstances.

The word "infection," from the Latin verb inficio, means literally to put or dip into anything; but in its accepted technical sense the putting of non-living particles into a living body does not constitute infection. A man who receives a load of bird-shot in the muscles of his thigh is not infected with bird-shot or with lead. But when a living micro-organism is introduced into the body of a living animal and multiplies there, the animal is infected. This infection may be localised, as in the case of a carbuncle, an abscess, a pneumonia, a pleurisy, etc., or it may be a general blood infection, as in relapsing fever, yellow fever, or the malarial fevers. In cholera and dysentery the infectious agent is in the alimentary canal, and penetrates to a greater or less extent the mucous membrane of the intestine. In typhoid fever it invades the glands of the intestine and mesentery, and the spleen. In diphtheria the mucous membrane of the throat and posterior nares is the usual seat of the infection.

Certain infectious diseases have their seat in various favourite localities upon the external portion of the body. These are the infectious skin diseases, 
which are also contagious for evident reasons. The fact that the infecting parasite must penetrate the body of its living host is well illustrated by the infectious skin disease known popularly as "itch" (scabies). The itch insect deposits its ova and rears its family in burrows beneath the epidermis, and thus becomes the agent in the production of an infectious skin disease. Certain other parasites, known as pediculi, infest the surface of the body, especially in localities covered with hair. As these do not penetrate the skin, their presence does not constitute an infection.

The fact that a disease may be transmitted through a series of individuals either by contagion or in some other way-by inoculation, by contaminated drinking-water, etc.-is evidence that it is due to a living disease germ of some kind and that it is consequently infectious. An individual who has been stung by a wasp or bitten by a rattlesnake is not infected but poisoned. The symptoms resulting from such a bite cannot be reproduced in another individual by inoculation of blood or other material from the body of the person bitten. But the bite of a rabid dog gives rise to an infectious disease- " hydrophobia "- which may be transmitted by inoculation through a series of men or dogs or other susceptible animals.

The poison introduced by a wasp or rattlesnake 
does not multiply in the body of the individual bitten and the symptoms produced bear a direct relation to the amount injected. Moreover, the symptoms follow very closely after the bite. On the other hand, an infectious disease resulting from inoculation, or contracted in any other way, is not developed at once; but after the introduction of the infectious material a certain interval elapses, technically known as the "period of incubation," before the symptoms characteristic of the disease are manifested. In the case of hydrophobia, resulting from the bite of a rabid animal, this so-called period of incubation may be greatly prolonged. It is seldom less than two weeks and may be six months or more. But, as a rule, the period of incubation is quite definite for each infectious disease, although differing greatly in different diseases. Thus it is usually less than three days in scarlet fever and diphtheria; from two to five days in yellow fever and influenza; from seven to ten days in whoopingcough; eleven or twelve days in smallpox; fourteen days in measles; and from seventeen to twenty-one days in mumps. In wound infections, resulting from the introduction of certain well-known disease germs into wounds produced by the surgeon's knife or otherwise, the period of incubation is comparatively short, and erysipelas or "blood-poisoning" may be developed within a few hours after the inoculation occurs. 
What has already been said will, it is hoped, make the following definition of an infectious disease quite clear. An infectious disease is one which is caused by the introduction of living disease germs within the body of a susceptible individual. This definition includes the idea of the reproduction-that is, multiplication within the body-of the specific disease germ, which must be living and thus capable of reproduction in order to produce an infectious disease. Moreover, it must find conditions favourable for its reproduction within the body or it will not give rise to any disease process-that is, the individual must be susceptible. 


\section{CHAPTER II}

\section{DISEASE GERMS}

$\mathrm{H}^{\mathrm{AVING}}$ ascertained that infection results from the introduction of living "disease germs" into susceptible individuals, it will be well to give some consideration to these agents of infection. The term disease germ is a popular one and is used to designate any micro-organism capable of giving rise to an infectious disease. The word micro-organism, which I shall have frequent occasion to use, may require a little explanation. By an organism we mean an organic structure which has been built up by vital processes. It may be a plant or an animal, it may be complex or simple, large or small, but it must, at one time at least, have been endowed with life. A microorganism is simply a microscopic organism, and being microscopic it is an organism of very simple structure, usually consisting of a single cell (" unicellular microorganism"). When using the word micro-organism with reference to a disease germ, we must use an 
adjective to indicate that the particular micro-organism under consideration is capable of producing disease, for there are numerous micro-organisms which are entirely harmless. The word pathogenic literally means disease-producing; a pathogenic micro-organism is therefore a microscopic organism capable of producing disease. This sounds a little more scientific than "disease germ," and it has seemed to me necessary to spend a little time in explaining the meaning of the term, as I may have occasion to use it from time to time, although it is my intention to avoid the use of technical terms as far as possible.

When we say that a certain infectious disease is due to a pathogenic micro-organism we have not committed ourselves as to the characters of this disease germ. It may belong to the animal or the vegetable kingdom; it may be round or oval or spiral in form; it may be large or small, although microscopic. But when I speak of the micrococcus of pneumonia or the bacillus of typhoid fever, I am using terms which convey much more definite information with reference to the disease germs referred to, and before proceeding any farther it will be desirable to make the reader acquainted with the principal characters of some of the best-known pathogenic micro-organisms. Some of these belong to the animal and some to the vegetable kingdom. Although so very minute and 
simple in structure, consisting, as a rule, of a single cell, they may be differentiated by the expert without great difficulty, and classified as animal micro-organisms ("Protozoa") or as vegetable micro-organisms ("Protophyta"). By far the greater number of known disease germs are recognised as vegetable micro-organisms belonging to the class known as Bacteria. This class includes a large number of harmless species, which abound especially in surface waters and in the upper layers of the soil.

The bacteria are classified with reference to their form. Those which are spherical are called micrococci; those which are longer in one diameter than in the other-oval, rod-shaped, or filamentous-are called bacilli; those which are elongated and spiral in form, like a corkscrew, are called spirilla (singular-micrococcus, bacillus, spirillum). The germs of pneumonia, of erysipelas, of boils and abscesses, of Malta fever, of cerebro-spinal meningitis, and some others are micrococci, all having distinct specific characters by which they can readily be recognised by an expert bacteriologist. The germs of typhoid fever, of tuberculosis, of influenza, of diphtheria, of dysentery, of bubonic plague, of tetanus, and of several infectious diseases of the lower animals (hog cholera, swine plague, anthrax, glanders) are bacilli. As in the case of the pathogenic micrococci, these all have 
specific characters by which they can be differentiated one from the other, independently of the fact that each gives rise to a specific infectious disease. The germs of Asiatic cholera and of relapsing fever are spirilla.

All bacteria multiply by binary division - that is, one cell divides into two, each resembling in form and dimensions the parent cell, and each in its turn dividing in the same way. The rapidity of multiplication by binary division varies greatly in different species, and depends upon circumstances relating to temperature, moisture, and suitable nutrient material. Under favourable conditions bacilli have been observed to divide in twenty minutes, and, as each daughter cell is equal in size to the mother cell, it is evident that an amount of nutrient material has been assimilated during this time equal to the quantity contained in the original cell. As a result of this rapid development, "colonies" containing millions of bacilli may be developed from a single cell in twentyfour to forty-eight hours. A simple calculation will show what an immense number of cells may be produced in this time as a result of binary division occurring, for example, every hour. The progeny of a single cell would be at the end of twenty-four hours 16,777,220. During the process of multiplication by binary division, the bacterial cells often remain attached to each other, and we may see them under 
the microscope grouped in pairs, or in chains, or in irregular masses.

Some of the bacteria multiply not only by binary division but also by the formation of spores, which correspond, so far as the preservation of the species is concerned, to the seeds of higher plants. The growing cells are delicate plants which are easily killed by heat and by various chemical agents (disinfectants). But the spores resist a much stronger solution of germicidal agents and a much higher temperature. They also resist desiccation, and may retain their vitality for months or years until circumstances are favourable for their development, when, under the influence of heat and moisture, they reproduce the minute microscopic plant-bacillus or spirillum-and multiplication by binary division again occurs. It is fortunate that comparatively few pathogenic bacteria produce spores, for if this were the case it would be a much more difficult task to arrest the progress of an epidemic of such diseases as typhoid fever, bubonic plague, cholera, or diphtheria. The only infectious disease of man in which spores have been demonstrated to be formed is tetanus, or lockjaw. As this disease is not likely to be communicated by the sick to those associated with them, either directly or indirectly, the formation of spores by the tetanus bacillus is not so serious a matter. 
As to the structure of the bacterial cells but little can be said, except that these simple, unicellular plants consist of a transparent protoplasm enclosed in a cellular envelope or membrane. The very varied characters which distinguish different species of bacteria make it evident that there are essential differences in the living cell contents, or protoplasm, although these differences are not revealed by chemical analysis or by our optical appliances. 


\section{CHAPTER III}

\section{CHANNELS OF INFECTION}

DISEASE germs gain access to the bodies of susceptible individuals, giving rise to infectious diseases, through various channels. The most important of these are doubtless the alimentary and respiratory tracts, to which access is obtained through the mouth and nasal passages. It is sometimes difficult to ascertain whether infection has occurred as a result of the deposition of germs contained in inspired air upon the mucous membrane of the respiratory passages or by reason of their having been taken into the stomach with food or drink.

Germs suspended in the air would be to a considerable extent deposited upon the moist mucous membrane of the mouth and nasal passages, and would be carried thence to the stomach rather than to the lungs. However, in certain diseases, infection no doubt results from the deposition of germs in the bronchial tubes. This is true of pulmonary consumption, of 
influenza (la grippe), of the pulmonic form of bubonic plague, of "wool-sorter's disease" (pulmonary anthrax), and of pneumonia. In diphtheria the initial infection commonly occurs upon the surface of the tonsils. This is also, no doubt, true in the various forms of tonsillitis and possibly in scarlet fever. Indeed there is good reason to believe that the tonsils constitute the avenue through which infection occurs, occasionally at least, in a considerable number of diseases of this class, including tuberculosis. The exact knowledge which has been gained during the past twenty years has made it evident that infection through the medium of the air is by no means as common as was formerly believed. We now know that malarial fevers and yellow fever are not contracted in this way, but that they result from inoculations made by infected mosquitoes. Some disease germs are quickly killed by desiccation and exposure to sunlight. These are not likely to be carried through the air in a living condition, and consequently the diseases produced by them are not propagated in this way. This is true of Asiatic cholera and to a considerable extent of typhoid fever, which diseases are recognised as being essentially water-born. However, the bacillus of typhoid fever resists desiccation for some time, and when the surface of the ground becomes contaminated with the discharges of typhoid- 
fever patients the bacillus may be carried by the wind, with dust, and deposited upon the moist mucous membrane of the mouths and nasal passages of individuals who breathe this dust-laden air. This is also true of the bacillus of bubonic plague, and to a still greater degree of the bacillus of tuberculosis.

The bubonic-plague bacillus, contained in the excreta of infected individuals and of rats, which are very susceptible to the disease, may retain its vitality for a considerable time when deposited upon the ground, and it is in this way that insanitary localities become centres of infection. The tubercle bacillus, which is contained in the matter coughed up from the lungs by persons suffering from pulmonary consumption, may retain its vitality and infecting power for a long time after the expectorated material containing it has been dried and pulverised.

It is evident that such dust is likely to be carried by currents of air and deposited in the lungs of persons who are compelled to live in localities where such insanitary conditions prevail. No doubt this is the usual way in which pulmonary consumption is contracted. Again, the dust deposited in the mouth and nasal passages may be swallowed and other forms of tubercular disease result - tubercular peritonitis, tubercular meningitis, tubercular joint disease, tuberculosis of the vertebral column. 
In smallpox, scarlet fever, and the "eruptive fevers" generally infectious material is given off from the surface of the body of the sick person. This is associated with cast-off epithelium, pus cells, etc., and constitutes a kind of dust which abounds in the sickroom and clings to the clothing and bedding of the patient and of those in attendance upon him. In influenza and whooping-cough the patient forcibly ejects small masses of mucus which soon become desiccated and are likely to make up a portion of the dust in apartments occupied by such patients. Evidently the great danger from infection in these diseases results from visiting the sick-room or handling clothing which has been exposed to contamination by infectious material coming from the body of the sick person.

In typhoid fever, Asiatic cholera, and dysentery the infectious material coming from the sick person is contained in the discharges from the bowels and is usually quickly removed from the sick-room. The great danger as regards the spread of these diseases consists in the possibility that ignorant or careless persons may throw these discharges upon the ground or dispose of them in some way which makes it possible for the germs to be washed into a well or a running stream from which water is used for drinking purposes. But this is not the only way in which 
disease germs may find their way from the excreta of the sick to the stomachs of healthy persons. If thrown upon the ground, flies alighting upon the foul material may subsequently visit a near-by kitchen and there walk over the food prepared for the family meal, leaving numerous typhoid bacilli in their tracks; or they may fall into the milk, or in some other unsuspected way convey the deadly microscopic germs to some article of food or drink. Again, articles of clothing soiled by the discharges of the sick may be the means of conveying infection to laundresses, who in handling such articles are liable to soil their hands or in some indirect way to introduce the pathogenic bacteria into their mouths. This mode of infection is liable to occur in any disease in which the germ is present in the discharges from the bowels, and especially in Asiatic cholera.

In Oriental countries, where human excreta constitutes a very common fertilising material, green vegetables, which are eaten raw, are believed to serve as the medium through which the germs of dysentery and cholera are occasionally conveyed to the stomachs of persons partaking of such articles of food. There is also considerable evidence in favour of the view that typhoid fever may be contracted by eating oysters which have been grown in sewage-polluted waters. The infectious disease known 
as trichinosis is contracted by eating pork containing living trichinæ. As these parasitic worms are promptly killed at a comparatively low temperature, cooked pork is quite harmless, so far as this disease is concerned.

Infection through wounds is far less common at the present day than was the case before aseptic surgery and the antiseptic treatment of wounds became established as a standard method of surgical procedure. Formerly epidemics of septicæmia, erysipelas, and hospital gangrene were of frequent occurrence, and the cleanest and best regulated hospitals were not exempt from these visitations. But in the light of our present knowledge such epidemics are no longer excusable and the infection of surgical wounds is extremely rare. Accidental wounds may, however, become infected at the time they are inflicted or because of failure to apply proper surgical dressings. Jagged and penetrating wounds which do not bleed are especially liable to be infected by the lodgment of germs in the deeper portion of the wound. It is in this way that tetanus or lockjaw is commonly produced. The tetanus bacillus forms spores which may retain their vitality for years. These are found in soil which has been enriched by manuring and in the dust of streets.

Bubonic plague is another disease which is com- 
monly contracted through accidental wounds. In countries where it prevails, it has been observed that the natives, who do not wear shoes and stockings, are much more liable to infection than Europeans, and it seems to be well established that infection may occur through insignificant wounds, such as scratches or abrasions of exposed parts of the body. We have also satisfactory evidence that tuberculosis may be transmitted to man by the accidental inoculation of an open wound. Malignant pustule, or anthrax, is communicated in the same way, and it sometimes happens that the inoculation is effected by flies which have been in contact with the infectious material escaping from the body of an animal having the disease or recently dead as a result of it.

It is well known that surgeons when operating upon an infected wound and pathologists when performing autopsies, in certain cases, are liable to a severe and sometimes fatal attack of "blood-poisoning" as a result of infection through a slight scratch or abrasion upon the hand, or through an accidental puncture made by a surgical needle. The germ which is most frequently concerned in this blood-poisoning, or septicæmia, is well known and is the usual cause of puerperal fever, erysipelas, and a considerable proportion of the cases of peritonitis. It is, therefore, in treating or making autopsies upon cases of this nature 
that physicians run the greatest danger of accidental infection.

The question whether infection may occur through the unbroken skin has been studied and an affirmative result obtained. The liability to infection in this way is, however, comparatively slight. When it does occur, it appears that the germs penetrate through the hair follicles. Infection may occur through mucous membranes, and it is in this way that infectious conjunctivitis and various other specific inflammations of mucous membranes are propagated. Infectious skin diseases, such as scabies (itch), ringworm, barber's itch, etc., may, no doubt, be contracted by susceptible persons, when conditions are favourable, independently of any wound or abrasion, especially in those who do not indulge in frequent bathing and thus give the germs time to penetrate the epidermis.

Researches made during the past few years have demonstrated that malarial fevers and yellow fever are communicated to man through the bites of infected mosquitoes. Certain infectious diseases of lower animals are also transmitted by insects. Thus it has been shown that ticks are responsible for the propagation of a fatal disease of cattle known as Texas fever, and an infectious disease of horses, which has recently prevailed extensively in the Philippine Islands, is communicated by a biting fly, which 
transmits the parasite from diseased to healthy animals - "surra disease." The tsetse-fly disease of Africa is transmitted in the same way and is very fatal to horses and also to the ox, the dog, the ass, and the sheep, but not to wild animals indigenous in the region where the tsetse fly is found. The parasite is present in great numbers in the blood of infected animals, and the fly simply acts as a carrier of this parasite from diseased to healthy animals. 


\section{CHAPTER IV}

\section{SUSCEPTIBILITY TO INFECTION}

$I^{\mathrm{T}}$ has long been known that certain infectious dis1 eases prevail only or principally among animals of a single species, while others are communicable to several species, including man himself. Thus typhoid fever, cholera, and relapsing fever are diseases of man, and during their epidemic prevalence none of the domestic animals contracts any of these diseases. On the other hand, the lower animals are subject to various infectious diseases which may prevail as fatal epidemics but which are never communicated to man-for example, chicken cholera, hog cholera, swine plague, rinderpest, foot-and-mouth disease. Again, several species, including man, may be susceptible to a disease while other animals have a natural immunity to it. Thus tuberculosis is common to man, to cattle, to apes, and to the small herbivorous animals (by inoculation), while the carnivora, as a rule, are immune; anthrax may be 
communicated by inoculation to man, to cattle, to sheep, to guinea-pigs, rabbits, and mice, but the rat, the dog, carnivorous animals generally, and birds are immune; glanders, which is essentially a disease of horses, may be communicated to man, to the guineapig, and to field-mice, while house-mice, rabbits, cattle, and swine are to a great extent immune; smallpox is essentially a disease of man, but a modified form of the disease may prevail among cattle (cowpox). Susceptibility to infection also depends to a considerable extent upon conditions relating to the individual. It is well known that an attack of certain infectious diseases protects the individual from subsequent attacks. This subject will receive attention in the chapter devoted to "Acquired Immunity." But in the absence of any such acquired immunity the susceptibility of individuals of the same species differs considerably, and the same individual may be more susceptible to infection at one time than at another. Certain families or races are especially susceptible to infection by certain disease germs. Thus the negro race is less susceptible to yellow fever and to the malarial fevers than the white race; on the other hand, smallpox is exceptionally fatal among negroes and dark-skinned races. In general it may be said that when an infectious disease is first introduced among primitive races, who, by reason of their 
isolation, have been previously exempt from it, it is apt to be exceptionally fatal. This is no doubt due to the fact that there has been no opportunity for the operation of the laws of natural selection, by "survival of the fittest." But under the operation of these laws, in process of time, a certain degree of race immunity is likely to be established.

Individual susceptibility depends to some extent upon age. As a rule, young animals are more susceptible to infection by inoculation than adults of the same species. In the human race we recognise certain diseases as especially liable to prevail among children-scarlet fever, whooping-cough, etc. It is also known that the tendency to tubercular infection diminishes with advancing years. Tubercular meningitis and tubercular joint diseases are most common in children, and pulmonary consumption in young adults. Again, the susceptibility of individuals depends to a considerable extent upon conditions relating to their general health. Various depressing agencies increase the susceptibility to infection. Most prominent among these are insufficient food, insanitary surroundings, great fatigue, and mental worrygrief, fear, etc. The fact that pestilence and famine are likely to go hand in hand has long been known. Whether the prevailing epidemic be cholera, bubonic plague, relapsing fever, typhus, or smallpox, the 
influence of insufficient food is most marked, and in times of distress, due to failure of the food supply, any infectious disease is liable to exhibit a malignancy and fatal character, although under ordinary conditions it may be comparatively harmless. Insanitary surroundings, by vitiating the air, insufficient ventilation and overcrowding of dwellings, factories, schoolhouses, etc.-all have a tendency to lower the vital resisting power of individuals subjected to such influences and to increase the susceptibility to infection. Debility resulting from loss of blood or the exhaustion following great fatigue also increases the susceptibility to various infectious diseases. Clinical observation shows that a similar result follows the excessive use of alcoholic drinks.

Localised infectious processes are not only more liable to be established in individuals whose vital energy is reduced by any of the causes mentioned, but also as a result of causes which reduce the resisting power of the tissues at the point of invasion. Thus a carbuncle or an abscess may develop in tissues that have been bruised or injured in any way; and the congestion or inflammation of the fauces which is so common as a chronic or acute condition -a "sore throat"-no doubt increases the susceptibility to diphtheritic infection in this locality. It is well known that pneumonia often follows attacks of 
measles, of influenza, or of bronchitis, in which diseases there is a catarrhal inflammation of the bronchial tubes which appears to favour infection by the specific micrococcus which is the usual cause of pneumonia. The victims of chronic alcoholism are especially subject to pneumonia.

Certain occupations increase the susceptibility to certain infectious diseases. Thus pulmonary consumption is more likely to be developed in those who lead an indoor life, whose occupation has a tendency to prevent full expansion of the lungs-tailors, seamstresses; and in persons who are compelled to breathe a dust-laden atmosphere-factory hands, grinders, etc. Susceptibility to pneumonia, influenza, tonsillitis, and diseases of the air passages generally is increased by living in over-heated apartments. Exposure to cold, per se, is not likely to increase the susceptibility to such infections in individuals who are habituated to living in the open air, such as sailors, hunters, soldiers living in tents, etc. 


\section{CHAPTER V}

\section{DISINFECTION}

THE object of disinfection is to prevent the extension of infectious diseases by destroying the specific infectious agent (germ) which gives rise to them. This is accomplished by the use of disinfectants.

The writer, as chairman of the Committee on Disinfectants of the American Public Health Association, in 1885, defined a disinfectant as "an agent capable of destroying the infecting power of infectious material."

In the preliminary report of this Committee the following general statements with reference to disinfection and disinfectants are also made:

"There can be no partial disinfection of such material [that is, material containing disease germs]; either its infecting power is destroyed or it is not. In the latter case there is a failure to disinfect. Nor can there be any disinfection in the absence of infectious material.

"Popularly, the term disinfection is used in a much broader 
sense. Any chemical agent which destroys or masks bad odours, or which arrests putrefactive decomposition, is spoken of as a disinfectant. And in the absence of any infectious disease it is common to speak of disinfecting a foul cesspool, or a bad-smelling stable, or a privy vault.

"This popular use of the term had led to much misapprehension, and the agents which have been found to destroy bad odours-deodorisers,-or to arrest putrefactive decompositionantiseptics, - have been confidently recommended and extensively used for the destruction of disease germs in the excreta of patients with cholera, typhoid fever, etc.

" The injurious consequences which are likely to result from such misapprehension and misuse of the word disinfectant will be appreciated when it is known that recent researches have demonstrated that many of the agents which have been found useful as deodorisers, or as antiseptics, are entirely without value for the destruction of disease germs.

" This is true, for example, as regards the sulphate of iron, or copperas, a salt which has been extensively used with the idea that it is a valuable disinfectant. As a matter of fact, sulphate of iron in saturated solution does not destroy the vitality of disease germs, or the infecting power of material containing them. This salt is, nevertheless, a very valuable antiseptic, and its low price makes it one of the most available agents for the arrest of putrefactive decomposition.

"Antiseptic agents also exercise a restraining influence upon the development of disease germs, and their use during epidemics is to be recommended when masses of organic material in the vicinity of human habitations cannot be completely destroyed, or removed, or disinfected.

"While an antiseptic agent is not necessarily a disinfectant, all disinfectants are antiseptics; for putrefactive decomposition is due to the development of 'germs' of the same class as that to which disease germs belong, and the agents which destroy the latter also destroy the bacteria of putrefaction, when brought in contact with them in sufficient quantity, or restrain their development when present in smaller amounts. 
"A large number of the proprietary 'disinfectants' so-called, which are in the market, are simply deodorisers or antiseptics of greater or less value, and are entirely untrustworthy for disinfecting purposes."

The offensive gases given off from decomposing organic material are no doubt injurious to health; and the same is true, even to a greater extent, of the more complex products known as ptomaines, which are a product of the vital processes attending the growth of the bacteria of putrefaction and allied organisms. It is therefore desirable that these products should be destroyed; and, as a matter of fact, they are neutralised by some of the agents which we recognise as disinfectants, in accordance with the strict definition of the term. But they are also neutralised by other agents-deodorants-which cannot be relied upon for disinfecting purposes, and by disinfectants, properly so-called, in amounts inadequate for the accomplishment of disinfection. Their formation may also be prevented by the use of antiseptics. From our point of view, the destruction of sulphuretted hydrogen, of ammonia, or even of the more poisonous ptomaines, in a privy vault, is no more disinfection than is the chemical decomposition of the same substances in a chemist's laboratory. The same is true as regards all of the bad-smelling and littleknown products of decomposition. None of these 
is "infectious material," in the sense in which we use these words; that is, they do not, so far as we know, give rise directly to any infectious disease. Indirectly they are concerned in the extension of the "filth diseases," such as cholera, bubonic plague, and typhoid fever. This because persons exposed to the foul emanations from sewers, privy vaults, and other receptacles of filth have their vital resisting power lowered by the continued respiration of an atmosphere contaminated with these poisonous gases, and are liable to become the victims of any infectious disease to which they may be exposed. 


\section{CHAPTER VI}

TESTS OF DISINFECTION

$W^{\text {HAT means have we of proving that the infect- }}$ ing power of infectious material has been destroyed?

Evidence of disinfection may be obtained: (a) from the practical experiments-experience-of those engaged in sanitary work; (b) by inoculation experiments upon susceptible animals; (c) by experiments made directly upon known disease germs.

(a) It is a matter of common experience that, when a room has been occupied by a patient with an infectious disease, such as smallpox, scarlet fever, or diphtheria, susceptible persons are liable to contract the disease weeks or even months after the patient has been removed from it, unless in the meantime it has been disinfected. If a second case does occur from exposure in such a room, it is evident that it has not been disinfected. But the non-occurrence of subsequent cases cannot always be taken as evidence 
that the means of disinfection resorted to were efficient. Negative evidence should be received with great caution. In the first place, the question as to whether susceptible individuals have been fairly exposed in the disinfected room must be considered. Then it must be remembered that susceptible persons do not always contract a disease, even when they are exposed in a locality known to be infected. A further difficulty in estimating the value of evidence obtained in practice arises from the fact that in connection with the special means of disinfection resorted to, such as fumigation, hanging up cloths saturated with a disinfecting solution, etc., it is customary to resort to additional precautionary measures, such as washing surfaces with soap and hot water, whitewashing plastered walls, and free ventilation. It is apparent that under these circumstances it would be unsafe to accept the fact that no other cases occurred in a room treated in this way as evidence that the particular disinfectant used is efficient for the destruction of the infectious agent of the disease in question. The fond mother who attaches a charm to her child's neck to protect it from evil also takes the precaution of guarding it from contact with other children who are sick with any infectious disease. If her child fortunately grows to manhood or womanhood without having suffered an attack of scarlet fever or diphtheria, she 
may imagine that her charm has protected it; but the evidence upon which her faith is founded is not of a nature to convince those who are familiar with scientific methods of demonstration. "Well educated" persons are often ready to testify in favour of methods of disinfection or of treatment upon evidence which, from a scientific point of view, has no more value than that which the fond mother in question has to offer in favour of the little bag containing camphor or assafœtida or some other charm of equal value which she has attached to her child's neck to keep it from catching scarlet fever or diphtheria at school. On a par with these charms, so far as disinfection is concerned, we may place the saucer of chloride of lime, which it was formerly the fashion to place under the bed of a patient sick with an infectious disease, the rag saturated with carbolic acid or chloride of zinc, suspended in the sick-room, and even the fumigations with burning sulphur, as sometimes practised by those who are unfamiliar with the evidence as to the exact value of this agent and the conditions necessary to insure successful disinfection with it.

Chloride of lime, sulphurous-acid gas, and carbolic acid are among our most useful disinfecting agents; but disease germs cannot be charmed away by them any more than by a little bag of camphor. 
Having pointed out the fact that negative evidence, in a restricted field of observation, must be accepted with great caution in estimating the value of disinfectants, we hasten to say that the combined experience of sanitarians, derived from practical efforts to restrict the extension of infectious diseases, is of the greatest value, and that this experience is, to a great extent, in accord with the results of exact experiments made in the laboratory.

(b) Inoculation experiments upon susceptible animals, made directly with infectious material which has been subjected to the action of a disinfectant, have been made by numerous observers. The proof of disinfection in this case is failure to produce the characteristic symptoms which result from inoculation with similar material not disinfected. Thus, Davaine found that the blood of an animal just dead from the disease known by English writers as anthrax or splenic fever, inoculated into a healthy rabbit or guinea-pig, in the smallest quantity, infallibly produces death within two or three days; and the blood of these animals will again infect and cause the death of others, and so on indefinitely. This anthrax blood therefore is infectious material, which can be utilised for experiments relating to the comparative value of disinfectants. Davaine made many such experiments, not only with the blood of anthrax, but also with that 
of a fatal form of septicæmia in rabbits, which is known by his name. Other investigators have followed up these experiments upon infectious material of the same kind, and also upon material from other sources - the infectious material of glanders, of tuberculosis, of symptomatic anthrax, of fowl cholera, of swine plague, etc.

It has been proved that the infectious agent in all of the diseases mentioned is a living germ, and that disinfection consists in destroying the vitality of this germ. But in experiments made with blood or other material obtained directly from diseased animals, the results would be just as definite and satisfactory if we were still ignorant as to the exact nature of the infecting agent. The test shows the destruction of infecting power without any reference to the cause of the special virulence, which is demonstrated to be neutralised by certain chemical agents in a given amount. All of the experiments made with the above-mentioned kinds of virus have been made upon the lower animals; but there is one kind of material which it is justifiable to use upon man himself, and with which numerous experiments of a very satisfactory character have been made. This material is vaccine virus. Fresh vaccine, when inoculated into the arm of an unvaccinated person, gives rise to a very characteristic result - the vaccine vesi- 
cle. The inference seems justified that any agent which will neutralise the specific infecting power of this material will also neutralise the smallpox virus. In these experiments the more careful investigators have taken the precaution of vaccinating the same person with disinfected and non-disinfected virus from the same source. A successful vaccination with the non-disinfected virus shows that the individual is susceptible and the material good; failure to produce any result is evidence that the potency of the disinfected virus has been destroyed by the chemical agent to which it was exposed.

(c) As already stated, it has been demonstrated that the infectious diseases of the lower animals, which have furnished the material for experiments upon disinfectants by the method of inoculation, are "germ diseases," and that the infectious agent is in each case a living micro-organism, belonging to the class known under the general name of Bacteria. The bacteria are vegetable organisms, which, by reason of their minute size and simple organisation must be placed at the very foot of the scale of living things; but they make up in number and in rapidity of development for their minute size.

Many of these disease germs are now known to us, not only by microscopic examination of the blood and tissues of infected animals, but also by "culture 
experiments." That is, we are able to cultivate them artificially in suitable media, and to study their mode of development in the laboratory, quite independently of the animals from which our "pure cultures" were obtained in the first instance. The culture fluids used are prepared from the flesh of various animals; and when to one of these a certain quantity of gelatine is added, we have a "solid culture medium," upon the surface of which some of these germs will grow most luxuriantly. To start such a "culture," it is only necessary to transfer, with proper precautions, a minute quantity of the infectious material to the surface of our culture medium, or into a fluid which has been found to be suitable for the growth of the particular organism which we desire to cultivate. A second culture is in the same way started from the first, and so on indefinitely.

Now it is evident that these pure cultures furnish us a ready means for testing the power of various chemical agents to destroy the vitality of known disease germs, as shown by their failure to grow in a suitable culture medium after exposure for a given time to a given percentage of the disinfectant. Very many experiments of this nature have been made. We may say here, that the experimental data on record indicate that those agents which are efficient for the destruction of any one of the pathogenic 
organisms upon which experiments have been made, or of harmless species of the same class, are efficient for the destruction of all, in the absence of spores. There is, it is true, within certain limits, a difference in the resisting power of different organisms of this class to chemical agents. This is not, however, sufficiently marked to prevent the general statement that a disinfectant for one is a disinfectant for all, in the absence of spores.

The last clause of the above statement calls for an explanation, and certain details with reference to the mode of reproduction of disease germs. All of the bacteria multiply by binary division; that is, one individual divides into two, and each member of the pair again into two, and so on. The spherical bacteria, known as micrococci, multiply only in this way, but some of the rod-shaped bacteria, or bacilli, also form spores. These spores correspond with the seeds of higher plants. They are highly refractive, oval or spherical bodies, which, under certain circumstances, make their appearance in the interior of the rods, which cease to multiply by binary division when spore formation has taken place. The point of special interest with reference to these spores is, that they have a resisting power to heat, and to the action of chemical disinfectants, far beyond that which is possessed by micrococci, or by bacilli without spores. The 
difference may be compared to the difference between a tender plant and its seeds to deleterious influences, such as extremes of heat and cold. Thus the spores of certain species of bacilli withstand a boiling temperature for several hours, while a temperature of $150^{\circ}$ Fahr. quickly kills most bacteria in the absence of spores. A similar difference is shown as regards the action of chemical agents. Certain agents, e. g., sulphurous-acid gas and carbolic acid,-which are extensively used as disinfectants, have been proved by exact experiments to be quite impotent for the destruction of spores. This being the case, it is advisable, in practical disinfection, always to use an agent which has the power of destroying spores, in those cases in which the exact nature of the disease germ has not been demonstrated. The cholera germ of Koch does not form spores; and there is good reason to believe that the same is true as regards the germs of yellow fever, of scarlet fever, and of smallpox, which have not yet been demonstrated. This inference is based upon evidence obtained in the practical use of disinfectants, and upon certain facts relating to the propagation of these diseases.

A second general statement, which is justified by the experimental evidence on record, is, that agents which kill bacteria in a certain amount prevent their multiplication in culture fluids, when present in quan- 
tities considerably less than are required to completely destroy vitality.

An agent, therefore, which in a certain proportion and in a given time acts as a "germicide," in a smaller quantity may act as an antiseptic-i.e., may prevent putrefactive decomposition by restraining the development of the bacteria of putrefaction. Antiseptics also prevent or retard the development of pathogenic bacteria. It follows from this that germicides are also antiseptics; but the reverse of this proposition is not true as a general statement, for all antiseptics are not germicides. Thus alcohol, common salt, sulphate of iron, and many other substances which are extensively used as antiseptics, have scarcely any germicide power, even in concentrated solutions, and consequently would be entirely unreliable as disinfectants.

Practically, antiseptics may accomplish the same result in the long run as we obtain in a short time by the use of disinfectants. If, for example, we prevent the development of the germs of cholera, or of typhoid fever, in an infected privy vault, by the continued use of antiseptics, these germs will in time lose their ability to grow, when introduced into a suitable culture medium. But in the meantime there is always the possibility that some of them may escape, with the fluid contents of the vault, into the 
surrounding soil, and contaminate some well or stream from which drinking-water is obtained. For this reason privy vaults, cesspools, and sewers should never be allowed to become infected. All infectious material, such as the dejections of patients with cholera or typhoid fever, should be destroyed at its source, in the sick-room; or, if it is ascertained that such material has been thrown into a privy vault, the entire contents of the vault should be promptly disinfected. The same rule applies to infectious material thrown upon the ground, or wherever it may be. 


\section{CHAPTER VII}

\section{DISINFECTION BY HEAT}

$\mathrm{IT}^{\mathrm{T}}$ is hardly necessary to say that burning of infectious material, infected clothing, etc., is an effectual method of disposing of it. This method of disinfection is always to be recommended, when practical and consistent with a due regard for economy and the rights of individuals. As a rule, articles of little value, which have been soiled with infectious material, had better be burned; and this is especially true of old clothing and bedding. But we have other efficient methods of disinfection, which make it unnecessary to sacrifice articles of value except under unusual circumstances.

While all disease germs are readily killed by exposure for a short time to the temperature of boiling water, many of the most important pathogenic bacteria are quickly destroyed by a much lower temperature than this - that is, when exposed in a liquid or in a moist condition. When in a desiccated condition, 
or exposed to the action of hot, dry air, a much higher temperature is required. This fact must constantly be kept in view in carrying out practical measures of disinfection, and for this reason the disinfection of clothing, blankets, etc., by dry heat is rarely employed. At quarantine stations and municipal disinfecting stations disinfection by steam is relied upon to a great extent, and has been proved by experience to be superior to all other methods. The disinfection of bandages, instruments, and dressings of all kinds for the "aseptic" treatment of surgical wounds is also accomplished by exposure to moist heat (steam or boiling water).

In considering the value of heat as a disinfectant, we must take account of the very great difference in the resisting power of growing bacteria and of the reproductive elements formed by some of them, which are known as "spores."

The spores of certain bacteria found in surface water and in the soil may resist the temperature of boiling water or of live steam for several hours, but fortunately the spores of known disease germs have far less resisting power. In experiments made nearly twenty years ago, I found that the spores of the anthrax bacillus did not grow after exposure to the temperature of boiling water for four minutes.

As already stated, bacteria which do not form 
spores are quickly killed by a temperature considerably below that of boiling water. The exact thermal death-point of a considerable number of the most important disease germs was determined by the writer in a series of experiments made in 1885 . The cholera germ and the micrococcus of pneumonia were the least resistant of all those tested, and were destroyed by ten minutes' exposure to a temperature of $130^{\circ}$ Fahr. The typhoid bacillus was killed in the same time by a temperature of $140^{\circ}$. In general the statement may be made as a result of my own experiments and those of other investigators, that pathogenic bacteria which do not form spores are killed by ten minutes' exposure to a temperature of $140^{\circ}$ Fahr. (moist heat), with the exception of the tubercle bacillus, which requires a somewhat higher temperature $\left(160^{\circ}\right.$ Fahr.). The list of known disease germs which are killed by ten minutes' exposure to a temperature of $140^{\circ} \mathrm{Fahr}$. $\left(60^{\circ} \mathrm{C}\right.$.) includes the bacillus of typhoid fever, of diphtheria, of bubonic plague, of glanders, the micrococcus of pneumonia, of erysipelas and puerperal fever, of boils and abscesses, the spirillum of cholera and of relapsing fever. In addition to these known germs it has been determined that the same temperature destroys the infecting power of vaccine virus, and presumably of smallpox virus, of hydrophobia virus, and of certain 
other kinds of infectious material in which the specific germ has not yet been demonstrated.

While dry hot air is, as a rule, unreliable for the destruction of disease germs, certain bacteria are quickly destroyed by desiccation. This is true of the cholera spirillum and of the micrococcus of pneumonia. On the other hand, the bacillus of typhoid fever, the bacillus of diphtheria, the bacillus of tuberculosis and the bacillus of bubonic plague may retain their vitality for weeks, or even months, when in a desiccated condition. This is true also of the virus of smallpox and of scarlet fever.

Low temperatures do not destroy bacteria. They have been exposed to a temperature of $-87^{\circ} \mathrm{C}$., obtained by the evaporation of liquid carbonic acid, but when again brought under favourable conditions showed no diminution in their capacity for development. Repeated freezing and thawing has, however, a deleterious action. The typhoid bacillus may be killed in cultures which are frozen and thawed out at intervals of three days, by repeating the operation five or six times.

The facts stated in this chapter make it evident that heat constitutes the most generally useful agent for the destruction of infectious material. Any article of food or drink which has been recently brought to a temperature approaching that of the boiling- 
point is surely free from living disease germs dangerous to man.

All articles of clothing which have been subjected to the ordinary operations of the laundry are safely disinfected.

Vessels containing the infectious discharges of persons suffering from cholera, typhoid fever, etc., if thoroughly treated with boiling water may be disinfected, together with their contents. To make sure of this the quantity of boiling water used should be three or four times greater than the contents of the vessel, and from ten to twenty minutes should be given for the disinfecting action of the hot water.

Articles of bedding and clothing which would be injured by immersion in boiling water may be disinfected by exposure to steam in a properly constructed disinfecting chamber or "steriliser."

Clothing may also be disinfected by dry heat if freely exposed in a closed chamber to the action of hot dry air, at a temperature of $125^{\circ} \mathrm{C}$. for two hours. 


\section{CHAPTER VIII}

SUNLIGHT AS A DISINFECTANT

$W^{\text {HAT }}$ has been said in the preceding chapter with reference to the germicidal value of heat and desiccation would indicate the utility of exposing infected articles to sunshine in the open air, as has long been the custom in domestic sanitary practice. But it has been ascertained by carefully conducted experiments that such exposure has an additional value on account of the disinfecting action of the sunlight per se. As long ago as 1877 two English experimenters (Downes and Blunt) in a communication made to the Royal Society of London presented evidence showing that sunlight has an injurious effect upon bacteria, and that sterilisation of cultures in liquid media could be effected by prolonged exposure to direct sunlight. Since then many experiments have been made by different observers and the fact has been fully confirmed. Even the spores of certain bacilli are destroyed by long exposure to sun- 
light. The distinguished German bacteriologist, Dr. Robert Koch, reported, some years since, the results of his experiments with the tubercle bacillus. He found that the time required to kill this bacillus varies from a few minutes to several hours, depending upon the thickness of the layer exposed. Even diffused daylight exerts a certain germicidal action, although the time of exposure is very much longer-five to seven days for the tubercle bacillus. In the writer's experiments made in 1892 it was found that two hours' exposure to direct sunlight was fatal to the cholera spirillum suspended in a liquid medium.

The electric light, and even gas-light, have also a germicidal action upon certain disease germs, although very much less in degree than sunlight. It has been ascertained that the rays at the violet end of the spectrum have the greatest disinfecting power, while the red rays are comparatively inert.

The facts stated fully sustain the popular idea that the exposure of infected articles of clothing and bedding in the sun is a useful sanitary precaution. Repeated and prolonged exposure will, however, be necessary to ensure safety. In the case of such diseases as smallpox, diphtheria, and scarlet fever more speedy and reliable measures of disinfection will be required. Exposure to the sunlight is nevertheless a 
most useful and economical procedure and is to be commended as a routine practice in domestic sanitation, and also as an additional and supplementary precaution when infected articles have been subjected to the action of other disinfectants. 


\section{CHAPTER IX}

\section{DISINFECTION BY GASES}

$I^{T}$ is impracticable to disinfect the atmosphere of an occupied apartment; for any gaseous or volatile agent which would destroy disease germs suspended in the air would render it irrespirable. Moreover the air of the sick-room should be constantly renewed by ventilation, and there is far less reason for disinfecting it when the patient has been removed than while it is occupied, for then the air may be quickly renewed by opening doors and windows. As is well known, particles of dust suspended in the air of a room have a tendency to settle upon the floor, upon window ledges, etc., and infected particles from the patient's body will constitute a portion of this dust in such diseases as smallpox and scarlet fever, while in tuberculosis, diphtheria, influenza, and pneumonia expectorated material may become desiccated and constitute a portion of the dust.

Every effort should be made to prevent a room 
occupied by patients sick with an infectious disease from becoming infected. Carpets, stuffed furniture, curtains, and other articles difficult to disinfect should be removed at the outset. Indeed, nothing should be left in the room which is not absolutely required, and all furniture and utensils should be of such a character that they can be readily disinfected by washing with boiling water or with a disinfecting solution. Abundant ventilation and scrupulous cleanliness should be maintained, and a disinfecting solution should always be at hand for washing the floor, or articles in use, the moment they are soiled by infectious discharges.

Daily wiping of all surfaces-floors, walls, and furniture-with a cloth wet with a disinfecting solution is to be recommended. For this purpose a solution of chloride of lime ( 2 per cent.), or of carbolic acid ( 2 per cent.), or mercuric chloride ( $1: 1000)$, may be used.

By such precautions the infection of the sick-room may be prevented, especially in those diseases, such as cholera and typhoid fever, in which the infectious agent is not given off from the general surface of the body of the sick person.

If a complete disinfection of the room is required it is indispensable that it be first vacated. It will then be practicable to use certain gaseous disinfectants. 
The various so-called disinfectants which are often recommended to purify the air of the sick-room are, at the most, simply deodorisers of greater or less value, and are entirely unreliable for the destruction of disease germs under the conditions existing in an occupied apartment.

Disinfection of the vacated room consists in the destruction of all infectious particles which remain attached to surfaces, or lodged in crevices, in interstices of textile fabrics, etc. The object in view may be accomplished by thorough washing with a reliable disinfecting solution, but many sanitarians think it advisable to "disinfect the room" with a gaseous disinfectant, such as formaldehyd or sulphur dioxid. If "fumigation" with sulphur dioxid is resorted to, the directions given by the Committee on Disinfectants of the American Public Health Association should be followed : that is, three pounds of sulphur should be burned for every thousand cubic feet of air-space. At the end of from twelve to twenty-four hours, doors and windows should be opened, and the room freely ventilated. After this fumigation, all surfaces should be washed with a disinfecting solution (chloride of lime 2 per cent., carbolic acid 2 per cent., or mercuric chloride $1: 1000)$, and afterwards thoroughly scrubbed with soap and hot water. Plastered walls should be whitewashed. 
Experiments made during the past twenty years have shown that fumigation by burning sulphur is not by any means so reliable a method of disinfection as was formerly supposed. It has very little value unless the articles to be disinfected are in a moist condition. This may be effected by introducing steam into the room together with the sulphur fumes. There is a class of diseases, however, in which sulphur fumigation is a most valuable method of disinfection: I refer to yellow fever and the malarial fevers, in which diseases the infectious agent is transmitted by mosquitoes. Such mosquitoes, after filling themselves with blood from the sick person, hang about the room, attached to the ceiling, to window-curtains, etc., for the purpose of digesting their meal and supplying themselves with another when occasion offers. The room is infected because of the presence of these infected mosquitoes, which with the blood of the patient have taken in the disease germs present in such blood. Disinfection in such a case consists in the destruction of the infected mosquitoes, and this is very readily accomplished by means of sulphur fumigation. Owing to the superior germicidal power of formaldehyd and its non-toxic properties, this gas has to a considerable extent taken the place of sulphur fumigation for disinfecting purposes.

Formaldehyd is generated either by the application 
of heat to an aqueous solution of the gas (formalin) or by the oxidation of wood alcohol.

In making practical use of this agent a suitable apparatus will be required. For the disinfection of a room with its contents, freely exposed for surface disinfection, one pound of formalin should be volatilised for each thousand cubic feet of air-space-the time of exposure to the disinfecting action of the gas being not less than twelve hours. In the absence of any apparatus satisfactory results have been obtained by the Department of Health of the city of Chicago, as follows :

"Ordinary bed sheets were employed to secure an adequate evaporatory surface, and these, suspended in the room, were simply sprayed with a forty per cent. solution of formalin through a common watering-pot rose-head. A sheet of the usual size and quality will carry from one hundred and fifty to one hundred and eighty cc. of the solution without dripping, and this quantity has been found sufficient for the disinfection of one thousand cubic feet of space. Of course, the sheets may be modified to any necessary number. . . Surface disinfection was thorough, while a much greater degree of penetration was shown than that secured by any other method."

Formalin may also be used in the disinfection of rooms and their contents by spraying all exposed surfaces.

Experiments made by Kinyoun and others show that formaldehyd gas does not injure the colour or textile strength of fabrics of wool, silk, cotton or linen 
and that it has no injurious action upon furs, leather, copper, brass, nickel, zinc, polished steel or gilt work. Iron and unpolished steel are attacked by the gas.

As is the case with sulphur dioxid the germicidal power of formaldehyd is increased by the presence of moisture.

Other volatile and gaseous disinfectants have been used, but from a practical point of view those mentioned are the best. Chlorin is a powerful germicide in the presence of moisture, but its irritant and corrosive properties interfere with its usefulness as a disinfecting agent. 


\section{CHAPTER $\mathrm{X}$}

\section{VARIOUS CHEMICAL DISINFECTANTS}

IT is my intention in the present chapter to refer briefly to some of the most useful chemical disinfectants. The most potent germicide is not always the best disinfectant for practical use. Questions of cost, poisonous properties, injurious effects upon textile fabrics, etc., must be considered in selecting an agent for any special purpose.

The mineral acids are all active germicides when used in solutions of proper strength, and a one-percent. solution of sulphuric, nitric, or hydrochloric acid will quickly destroy pathogenic bacteria in the absence of spores. Such a solution could be safely used to disinfect the excreta of patients suffering from cholera or typhoid fever. Among the vegetable acids it is only necessary to mention citric acid, which has been recently recommended, in the form of lemon juice, for destroying typhoid bacilli in drinking-water. The idea that the addition of a spoonful of lemon-juice to 
a glass of water, just before drinking it, will be sufficient to ensure the destruction of typhoid bacilli present in the water is not well founded. Exact experiments show that the bacillus of typhoid fever is killed, in five hours' time, by a solution containing one-half of one per cent. of citric acid. But the time element must not be overlooked. However, the experimental evidence supports the view that the typhoid bacillus or the cholera spirillum would not retain their vitality very long in a strong lemonade, containing one per cent. or more of citric acid. The addition of sulphurous acid to water is still more effective, especially as regards the cholera germ, which is very sensitive to the action of acids. The addition of this acid to drinking-water during the prevalence of cholera has been recommended and practised, apparently with good results. It has been shown by carefully conducted experiments that one part in five hundred will destroy the cholera spirillum in the course of a few hours.

The caustic alkalies all have considerable germicidal value. Potash soap containing an excess of alkali will destroy the typhoid bacillus in six-per-cent. solution within thirty minutes; and the scrubbing of floors, articles of furniture, etc., with such a solution, especially when used hot, is a most reliable method of disinfection. Solutions of potash-common lye- 
or of soda are extremely valuable for certain purposes in domestic sanitation, and scientific researches fully justify the cleansing methods with these agents which have long been popular with good housewives. A hot solution of caustic soda or potash in the proportion of one part to two hundred of water will quickly destroy the germs of cholera, of typhoid fever, of diphtheria, or of glanders.

Caustic lime is also an excellent disinfectant and has the advantage of being comparatively cheap. For this reason it is one of the best agents for the disinfection of masses of filth in vaults or cesspools and upon the surface of the ground. Milk of lime, made by slaking fresh quicklime with water and mixing the resulting hydrate of lime with eight parts of water may be used for this purpose and also for the disinfection of liquid excreta in the sick-room. Lime wash applied to surfaces is a reliable disinfectant, as has been proved by experiment, thus giving scientific confirmation of the value of a method which has gained popular favour as a result of experience-that is, of the sanitary value of whitewash freely and frequently applied to outbuildings, cellar walls, etc.

Various coal-tar products have been proved to be valuable germicides, and on account of their comparative cheapness have been largely used in practical disinfection. Among these the most useful are 
carbolic acid, creolin, cresol, and lysol. A five-per-cent. solution of either of these may be used for the disinfection of the liquid discharges of patients with cholera, cholera infantum, dysentery, or typhoid fever; also for the expectoration of those suffering from pulmonary tuberculosis, pneumonia, diphtheria, influenza, scarlet fever, measles, or whooping-cough. It must be remembered, however, that time is an element in the accomplishment of disinfection, and after adding the disinfecting solution to the material to be disinfected an interval of an hour or more should be allowed before the contents of the vessel are thrown into a vault or sewer. A two-per-cent. solution of one of the above mentioned disinfectants may be used for washing floors, articles of furniture, leather, etc. Such a solution may also be used for the disinfection of pocket-handkerchiefs, bed-linen, underclothing, and other articles which require disinfection before sending them to the laundry. The articles to be disinfected should be completely immersed in the disinfecting solution, contained in a suitable receptacle, and left for at least an hour before removal from the sick-room or its immediate vicinity.

Chlorinated lime ("chloride of lime," "bleaching powder") is a valuable disinfectant and also a prompt deodoriser. It may be used for the disinfection of excreta in the sick-room, in open pits, etc., and for 
infected sputa. A solution containing six ounces of good bleaching powder to the gallon of water will be suitable for ordinary use. By exposure to the air the chloride of lime rapidly deteriorates in quality. It should therefore be kept in air-tight receptacles, and only so much of the disinfecting solution made as is required for immediate use. Owing to its bleaching properties and injurious action upon fabrics of all kinds the chlorinated-lime solution is seldom used for the disinfection of bed-linen and articles of clothing, but it may be employed for washing floors and other woodwork.

Many of the metallic salts have decided germicidal value, and some of them have been largely used in practical disinfection. Among these the bichloride of mercury, or "corrosive sublimate," has a prominent place. In very dilute solutions this salt is fatal to all known disease germs, and in the proportion of $\mathrm{I}: 500$ it will destroy the spores of pathogenic bacteria (anthrax, tetanus). For ordinary use a standard solution of one part in one thousand parts of water may be used. This will be suitable for washing surfaces and for the disinfection of bed- and body-linen. But owing to the fact that the bichloride of mercury combines with albuminous substances, and is thus rendered practically inert, this salt is not a reliable disinfectant for excreta or expectorated matters. The 
very poisonous nature of this salt must be constantly kept in mind by those who make use of it for disinfecting purposes. The solution is colourless, and a fatal dose might easily be mistaken for water. To avoid such accident it is customary to colour the solution with indigo or an anilin dye.

Sulphate of copper has been used to some extent, especially in France, for the same purposes as the salt last mentioned (corrosive sublimate). Its germicidal value is considerably less, but in solutions containing from two or five per cent. it is reliable for the destruction of pathogenic bacteria not containing spores. Like the bichloride of mercury its germicidal action is neutralised to a considerable extent by the presence of albuminous material. It is therefore not to be selected for the disinfection of sputa and excreta.

With reference to the various proprietary disinfectants which are in the market and largely used, I would say that many of them are deodorants of more or less value and are entirely unreliable for the destruction of disease germs. Others contain germicidal agents of value; but, as a rule, they are not economical in use, as compared with heat, carbolic acid, formaldehyd, and other disinfectants of established value referred to in the present volume. 


\section{CHAPTER XI}

NATURAL IMMUNITY

$I^{T}$ is hardly necessary to explain that absence of susceptibility to an infectious disease constitutes what is known as immunity for or against the disease in question. Now this immunity may be natural or acquired - that is, due to inheritance or developed in a susceptible individual subsequent to birth. We have said in a preceding chapter that man is immune as regards certain infectious diseases of the lower animals, and that many of the infectious diseases to which he is subject are not transmitted to the domestic animals with which he is most closely associated. This natural immunity is not, however, in all cases absolute and complete. For example, the white rat possesses a remarkable immunity against anthrax, a disease which may be communicated by inoculation to sheep, cattle, rabbits, guinea-pigs, mice, and to man himself. But it has been shown that this natural immunity of the white rat may be overcome by giving 
it an exclusively vegetable diet. Again, natural immunity may in some cases be overcome by the devitalising agencies mentioned in the chapter on susceptibility to infection (starvation, great fatigue, etc.). Infection also depends upon the comparative virulence of the infecting agent, or germ, and to some extent upon the number of germs introduced.

Immunity, therefore, whether natural or acquired, often has only a relative value, and may be overcome as a result of circumstances favourable to infection. Thus it has been found that germs having very little pathogenic virulence, and harmless under ordinary conditions, may kill guinea-pigs when injected into the muscles of the thigh after they have been bruised by mechanical violence. Pasteur found that fowls, which have a natural immunity against anthrax, become infected and die if they are subjected to artificial refrigeration after inoculation. Pigeons have a natural immunity against anthrax, but if they are enfeebled by lack of food they succumb to inoculations with the anthrax bacillus.

The pathogenic power of known disease germs also varies greatly as a result of conditions relating to their development. In general it may be said that cultivation in the bodies of susceptible animals increases the virulence of disease germs. Attenuation of virulence may be effected by several methods, all 
of which depend upon subjecting the germs to prejudicial influences of one kind or another-long exposure to oxygen, exposure to a temperature a little short of that which would completely destroy their vitality, exposure to various chemical agents.

Attenuated germs may cause infection in very susceptible animals, and may gain in virulence as a result of their growth in such animals. After passing through a series of susceptible animals they may finally acquire such pathogenic virulence that they can overcome the resisting power of animals having a considerable degree of natural immunity. Applying the facts ascertained by experiments upon the lower animals, we can understand how the earlier cases in an epidemic may occur in the most susceptible individuals, and are often comparatively mild; but, as a result of its transmission through a series of individuals, the germ gradually increases in virulence and the epidemic in malignancy. Thus the earlier cases in an epidemic of diphtheria or of scarlet fever are often mild, while later cases prove to be extremely difficult to manage and show a high rate of mortality.

Infection also depends to some extent upon the number of germs introduced. The resources of nature, upon which immunity depends, may be sufficient to dispose of a few typhoid bacilli or diphtheria 
bacilli; while a larger number introduced at one time may overwhelm the resisting power of the individual.

The essential difference between a susceptible and immune animal depends upon the fact that in one the pathogenic germ, when introduced by accident or experimental inoculation, multiplies and invades the tissues or the blood, where, by reason of its nutritive requirements and toxic products, it produces changes in the tissues and fluids of the body which constitute disease and may result in death. On the other hand, in an immune animal multiplication of the germ and consequent disturbance of vital functions does not occur, or is restricted to a local invasion of limited extent, in which the parasitic invader soon succumbs to the resources of nature. This essential difference evidently depends upon conditions favourable or unfavourable to the development of the germ; or upon its destruction by some active agent present in the tissues or fluids of the body of the immune individual ; or upon a neutralisation of its toxic products by some substance in the body of the animal which resists infection.

Among the unfavourable conditions which may be supposed to prevent the development of disease germs in animals which have a natural immunity against infection by them, we may mention, first, the temperature of the animal. It is well known that 
the constant body temperature of mammals varies considerably for different species. Birds, as a rule, have a higher temperature than mammals, and reptiles are "cold-blooded animals." A disease germ, like the tubercle bacillus, for example, which requires for its development a temperature not very different from that of a healthy man, may fail to infect a pigeon because of its comparatively high, or a frog because of its low, temperature. Certain experiments which have been made by bacteriologists give support to this view. This is the explanation offered by Pasteur of the immunity of fowls against anthrax - a disease of sheep and cattle; and in support of this view he showed by experiment that when chickens are refrigerated by being immersed in cold water, after inoculation, they are liable to become infected and to die. Again, the composition and especially the reaction of the blood and other body fluids may perhaps be the determining factor. Some germs do not grow readily in an alkaline medium; and some animals - for example, the white rat have a highly alkaline blood. Experiments made by the German bacteriologist, Behring, seem to show that the natural immunity of the white rat against anthrax infection is lost when the animal is given food which reduces the alkalinity of its blood. It is probable, also, that the presence or absence of 
various substances favourable or unfavourable to the development of particular disease germs may, in certain cases, be the fundamental cause of race immunity. It has been shown by experiment that natural immunity may be overcome in certain animals by inoculating them with disease germs mixed with certain chemical substances - or with sterilised cultures of various bacteria. The susceptibility of the victims of chronic alcoholism to infection by various pathogenic bacteria is well known. Whether this is due to the presence of alcohol or to chemical changes in the body fluids resulting from its use is not determined. A complete knowledge of the facts would probably show that immunity, natural or acquired, in the ultimate analysis, to a large extent has a chemical basis - that is, it depends upon the presence of some substance which exercises a deleterious influence upon the germ or neutralises its toxic products.

That the blood-serum of healthy animals contains substances which have a decided germicidal effect has been demonstrated by experiments made with blood withdrawn from the circulation. This property belongs to the clear serum which is obtained after coagulation of the fibrin and separation of the clot containing the red and white blood corpuscles. When the blood-serum is kept for some time it loses its germicidal activity. This is also destroyed by heat, 
but not by freezing. The blood of different species differs considerably in this regard, and that of the same species may show a decidedly greater germicidal action for one disease germ than for others. That the presence of these germicidal substances constitutes a most important element in natural immunity can scarcely be doubted. According to Behring the blood of the rat and of the frog, which animals have a natural immunity against anthrax, is especially fatal to the anthrax bacillus. The numerous experiments which have been made show that the germicidal action of blood-serum, which is very promptly manifested, is limited as to the number of bacteria which may be destroyed by a given amount. When the number of bacteria is excessive only a limited number are destroyed, and after an interval those not destroyed multiply abundantly in the bloodserum, which, in the absence of its germicidal constituent, is an excellent culture medium for many pathogenic bacteria.

It would appear from this that the element in the blood to which the germicidal action is due is neutralised in exercising this power-in other words, that the effect is the result of a chemical reaction. These germicidal substances in the blood of healthy animals are complex nitrogenous compounds, which belong to the group of organic bodies known to 
chemists as proteids. They are sometimes spoken of as "defensive proteids," because they appear to serve as a provision of nature for defence against disease germs. Possibly the increased susceptibility to infection resulting from starvation, great fatigue, and other devitalising agencies is due to a diminution in the quantity of these defensive proteids present in the blood. These germicidal substances differ from the "antitoxins," of which I shall speak in a subsequent chapter, in the fact that their power to destroy pathogenic bacteria is destroyed by a comparatively low temperature ( $140^{\circ}$ Fahr.). Independent researches made by several different investigators seem to show that the defensive proteids of the blood have their origin in the leucocytes, or white blood-corpuscles, and that an alkaline condition of the blood is favourable, if not essential, to the formation of such germicidal substances, or at least to their release from the leucocytes. The number of leucocytes increases in certain infectious diseases, and this increase, together with an increased alkalinity of the blood, which has been noted, may be a provision of nature for overcoming infection when it has already occurred.

A more direct rôle has been ascribed to the leucocytes as defenders of the living body against invasion by pathogenic bacteria. 
In my chapter on "Bacteria in Infectious Diseases," in Bacteria, published in the spring of 1884 , but placed in the hands of the publishers in I883, I say:

"If we add a small quantity of culture fluid containing the bacteria of putrefaction to the blood of an animal, withdrawn from the circulation into a proper receptacle, and maintained in a culture oven at blood-heat, we shall find that these bacteria multiply abundantly, and evidence of putrefactive decomposition will soon be perceived. But if we inject a like quantity of the culture fluid, with its contained bacteria, into the circulation of a living animal, not only does no increase and no putrefactive change occur, but the bacteria introduced quickly disappear, and at the end of an hour or two the most careful microscopical examination will not reveal the presence of a single bacterium. This difference we ascribe to the vital properties of the fluid as contained in the vessels of a living animal, and it seems probable that the little masses of protoplasm known as white blood-corpuscles are the essential histological elements of the blood, so far as any manifestation of vitality is concerned. The writer has elsewhere (188I) suggested that the disappearance of the bacteria from the circulation, in the experiments referred to, may be effected by the white corpuscles, which, it is well known, pick up, after the manner of amœbæ, any particles, organic or inorganic, which come in their way. And it requires no great stretch of credulity to believe that they may, like an amoba, digest and assimilate the protoplasm of the captured bacterium, thus putting an end to the possibility of its doing any harm.

"In the case of a pathogenic organism we may imagine that, when captured in this way, it may share a like fate if the captor is not paralysed by some potent poison evolved by it, or overwhelmed by its superior vigour and rapid multiplication. In the latter event the active career of our conservative white corpuscles would be quickly terminated, and their protoplasm would serve as food for the enemy. It is evident that in a contest of this kind the balance of power would depend upon circumstances relating 
to the inherited vital characteristics of the invading parasite and of the invaded leucocyte."

This explanation is now very commonly spoken of as the "Metschnikoff theory," although, as shown.by the above quotations, it was clearly stated by the writer several years ( I 88I) before Metschnikoff's first paper ( 1884 ) was published. Metschnikoff has, however, been the principal defender of this explanation of acquired immunity, and has made extensive and painstaking researches, as a result of which many facts have been brought to light which appear to give support to this theory.

The recorded experimental evidence leads us to the conclusion that natural immunity is partly due to germicidal substances present in the blood-serum, which have their origin in the leucocytes, and are soluble only in an alkaline medium; that local infection is usually resisted by an afflux of leucocytes to the point of invasion, which to some extent serve to protect the individual from disease germs, by their direct action as "phagocytes" — that is, by picking up and destroying the invading parasites. These agencies, together with conditions relating to body temperature, and the chemical constitution of the fluids and tissues of the body constitute the principal factors upon which natural immunity depends. 


\section{CHAPTER XII}

\section{ACQUIRED IMMUNITY}

$\mathrm{I}^{\mathrm{T}}$ is well known that in certain infectious diseases a single attack protects the individual from subsequent attacks. In some cases such protection lasts during life, while in others it is more or less temporary.

The protection afforded by an attack not only varies in different diseases, but in the same disease differs greatly in individual cases. Thus second or even third attacks of smallpox occasionally occur, although, as a rule, a single attack is protective.

In certain diseases second attacks are not infrequent. This is true of pneumonia, Asiatic cholera, diphtheria, and especially of influenza. But there is usually a considerable interval between two attacks of any of these diseases, and the inference is that temporary immunity results from each attack. In the malarial fevers, which are due to infection by a blood parasite of a different class, no immunity is afforded by an attack of the disease, in its usual form 
at least-chills and fever. On the other hand, the debility resulting from an attack seems to constitute a predisposition to subsequent attacks.

As showing the liability to two or more attacks from certain infectious diseases, I give below a table compiled from the literature, as given in medical journals, which was published several years ago (Maiselis).

$\begin{array}{lrcc}\text { Second } & \begin{array}{c}\text { Third } \\ \text { attacks. }\end{array} & \begin{array}{c}\text { Fourth } \\ \text { attacks. }\end{array} \\ \text { attacks. } \\ \text { Scarlet fever } & 505 & 9 & \circ \\ \text { Measles } & 29 & 4 & 0 \\ \text { Typhoid fever } & 36 & 1 & \circ \\ \text { Cholera } & 202 & 5 & \text { I } \\ \text { atla } & 29 & 3 & 2\end{array}$

These figures support the view generally entertained by physicians, that second attacks of measles are comparatively rare, while second attacks of smallpox are not infrequently observed. Considering the large number of cases of typhoid fever which occur annually in all parts of Europe and America, the number of second attacks reported is comparatively small, and in this disease it may be stated that, as in smallpox and scarlet fever, a single attack usually protects during life from subsequent attacks.

The second attacks of cholera recorded are not numerous, but an investigation made in the countries where this disease prevails annually, or frequently, would probably show that two or more attacks of the 
disease in the same individuals are not of infrequent occurrence.

That immunity may result from a comparatively mild attack as well as from a severe one is a matter of common observation in the case of smallpox, scarlet fever, yellow fever, measles, and other infectious diseases. And it not infrequently happens that such mild attacks are not recognised.

In that case the protection afforded during subsequent epidemics is often ascribed to natural immunity. This is no doubt the true explanation of the immunity of natives of Havana, and other cities where yellow fever has prevailed for many years, to this disease. An unrecognised attack suffered during childhood has resulted in immunity which is supposed to be due to inheritance. The popular idea that natives are exempt from this disease is an additional motive for calling it by some other name, especially as the attacks are usually extremely mild in nativeborn children.

The production of immunity by protective inoculations was for a long time limited to a single diseasesmallpox. Inoculations with virus, obtained from a pustule on a smallpox patient, were extensively practised before the discovery of vaccination by Jenner. These inoculations gave rise to a mild attack of the disease, followed by immunity, which was apparently 
as complete as that following a more severe attack contracted in the usual way. This method seems to have been practised by Eastern nations long before it was introduced into Europe. It was extensively employed in Turkey early in the eighteenth century, and was introduced into England through the influence of Lady Mary Wortley Montagu. No doubt the mortality from smallpox was greatly diminished by these inoculations; but they were attended by the disadvantage that the disease was propagated by them, inasmuch as inoculated individuals became a source of infection for others. Inoculation was still practised in England for some time after the demonstration of the protective value of vaccination, but in 1840 it was prohibited by an act of Parliament.

There is some evidence that vaccination as a protection against smallpox was practised to a limited extent prior to the time of Jenner. Thus Humboldt has stated that it was known at an early period to the Mexicans. But its introduction as a reliable method of protecting against smallpox is due to the patient researches of the renowned English physician, whose attention was first attracted to the subject in 1768 , although it was not until 1796 that he made his first vaccination in the human subject. His first public institution for the practice of vaccination was established in 1799 , and the following year the practice 
was introduced into France, Germany, and the United States.

In the infectious disease of cattle known as pleuropneumonia, protective inoculations were successfully made some time before the demonstration by Pasteur of the efficacy of such noculations in anthrax and chicken cholera (I880). Various methods have been amployed. The natives of the banks of the Zambeze cause animals to swallow a certain quantity of the liquid from the pleural cavity of an animal recently dead, and thus give them immunity. The virus has been injected into the circulation by some experimenters, and others have proposed to attenuate it by heat. But the method which has been most extensively employed is that discovered by the Dutch settlers at the Cape of Good Hope (the Boers), and consists in inoculating animals in the tail with serum from the lungs of an animal recently dead, or with a virus obtained from the tumefaction produced by such an inoculation in the tail. This is also the method most extensively employed in Australia, into which country infectious pleuro-pneumonia was introduced in 1858 .

Toussaint, a pioneer in researches relating to protective inoculations, has a short paper in the Comptes rendus of the French Academy of Sciences of July I 2, 1880, entitled "Immunity from Anthrax 
('charbon') Acquired as a Result of Protective Inoculations."

In this communication, he reports his success in conferring immunity upon five sheep by means of protective inoculations, and also upon four young dogs. We must, therefore, accord him the priority in the publication of experimental data demonstrating the practicability of accomplishing this result.

But it is especially to the experimental researches of Pasteur that we are indebted for the development of practical methods, which have been extensively employed in protecting cattle, sheep, and swine from the fatal effects of various infectious maladies, and man from hydrophobia as the result of the bite of a rabid animal.

Pasteur's inoculations are made with an "attenuated virus"-that is, with a culture of a pathogenic micro-organism which has a diminished degree of virulence and which wher introduced into a susceptible animal induces a non-fatal and comparatively mild attack.

The researches of Pasteur and of his followers in this line of investigation show that pathogenic virulence may be attenuated by prolonged exposure to oxygen; by exposure to a temperature a little below that which would completely destroy vitality; by the action of certain chemical agents; and, in some 
cases, by passing through a series of non-susceptible animals.

As a general rule, pathogenic virulence is increased by successive inoculations in susceptible animals and diminished by cultivating the pathogenic micro-organism in artificial media outside of the animal body, or by passing it through animals having but slight susceptibility to its pathogenic action. As pathogenic virulence depends, to a considerable extent at least, upon the formation of toxic substances during the active development of the pathogenic micro-organism, we infer that diminished virulence is due to a diminished production of these toxic substances.

An important step was made in the progress of our knowledge in this field of research when it was shown that animals may be made immune against certain infectious diseases by inoculating them with filtered cultures containing the toxic substances just referred to, but free from the living bacteria to which they owe their origin. The first satisfactory experimental evidence of this important fact was obtained by Salmon and Smith in I886. These bacteriologists succeeded in producing an immunity in pigeons against the pathogenic effects of the bacillus of hog cholera, which is very fatal to these birds, by inoculating them with sterilised cultures of the bacillus mentioned. Similar results were reported by Roux in 1888 , from 
the injection into susceptible animals of sterilised cultures of the anthrax bacillus. More recently (I 890) Behring and Kitasato have shown that animals may be made immune against the pathogenic action of the bacillus of tetanus or the bacillus of diphtheria by the injection of filtered, germ-free cultures of these bacilli.

In Pasteur's protective inoculations against hydrophobia, it is probable that the immunity which is developed after infection by the bite of a rabid animal is due to the toxin of this disease present in the emulsion of spinal cord which is used in these inoculations.

We have also experimental evidence that animals may acquire an artificial immunity against certain poisonous substances of animal and vegetable origin.

By inoculations with minute and gradually increasing doses, animals may be made immune against rattlesnake venom, and there is reason to believe that persons who have been repeatedly stung by poisonous insects - mosquitoes, bees - acquire a considerable degree of immunity from the distressing local effects of their stings.

Professor Ehrlich, of Berlin, in $189 \mathrm{I}$, published the results of some researches which have an important bearing upon the explanation of acquired immunity, and which show that susceptible animals may be 
made immune against the action of certain toxic proteids of vegetable origin, other than those produced by bacteria,-one, ricin, from the castor-oil bean, the other, abrin, from the jequirity bean. The toxic potency of ricin is somewhat greater than that of abrin, but both are far more poisonous than strychnin. A small quantity of a solution containing one part in one hundred thousand parts of water will quickly kill a mouse. But when injected into these animals in still smaller and non-fatal doses, or given to them with their food, immunity may be established to such a degree that they resist subcutaneous injections of two hundred to four hundred times the quantity required to kill a non-immune animal.

In a later paper (1892), Ehrlich has given an account of subsequent experiments which show that the young of mice, which have an acquired immunity for these vegetable poisons, acquire immunity from the ingestion of their mother's milk; and also that immunity from tetanus may be acquired in a brief time by young mice through their mother's milk.

These results have been confirmed by other observers and show that some substance upon which acquired immunity depends is present in the milk of an immune animal. 


\title{
CHAPTER XIII
}

\author{
ANTITOXINS
}

$W^{E}$ have seen in the preceding chapter that, in certain cases at least, acquired immunity is due to the presence of substances developed in the body of the immune animal which may escape in the milk of a nursing female and give protection to its young. Such protective substances are called "antitoxins," because it is evident that they, in some way, neutralise the toxins of various disease germs and the animal and vegetable poisons referred to-abrin, ricin, snake-venom.

The German chemists, Brieger and Ehrlich, have succeeded in separating the antitoxin of tetanus from the milk of a goat which had been made immune by repeated inoculations with the toxic products of the tetanus bacillus. A precipitate obtained from the milk by chemical processes proved to be from four hundred to six hundred times as active as the milk itself, as shown by its power to neutralise 
the tetanus toxin. But the usual source from which antitoxins are obtained for practical purposes is the blood of animals which have been rendered immune. The diphtheria antitoxin, which is now extensively and successfully employed for the cure of diphtheria, is obtained from the blood of horses which have been immunised by repeated inoculations with the toxic products of the diphtheria bacillus.

A most interesting question presents itself in connection with the discovery of the antitoxins: Is the animal which has been immunised against any particular toxin also immune for other poisonous substances of the same class? This question has been definitely answered in the negative by experimental investigation. In other words, each specific toxin causes the development in the body of the immune animal of a specific antitoxin which has no neutralising action upon any other toxin than that which gave rise to its production. An animal which has been immunised against the toxic action of ricin is poisoned by the usual fatal dose of abrin. The tetanus antitoxin affords no protection against the poisonous products of the diphtheria bacillus and vice versa.

The antitoxins protect susceptible animals from infection when introduced by inoculation at the same time or in advance of the disease germs against which they have a specific action. They may also, in 
some cases, be successfully used in the cure of infectious diseases, when these have not advanced too far.

The remarkable success attending the use of the diphtheria antitoxin for this purpose is well known and a certain degree of success has attended the efforts of physicians in the treatment of other dis. eases by the same method-tetanus, erysipelas, pneu. monia. But specific treatment by antitoxins is still in its infancy and much careful experimental work and clinical experience will be necessary in order to determine the practical value of this method in the diseases mentioned and in other infectious maladies. Enough is known at present, however, to lead to the hope that when methods have been devised for obtaining these various antitoxins in a pure and concentrated form they will constitute a most valuable addition to our resources for the treatment of infectious diseases. Indeed the only hope of specific medication for such diseases appears to lie in this direction.

In the present volume I shall not attempt to discuss the questions connected with the origin of the antitoxins in the bodies of immunised animals, the chemical nature of these substances, or the mode of their action in neutralising the toxins. These are questions which would involve a considerable amount of technical knowledge on the part of the reader for 
an understanding of the most advanced views regarding them, as expounded by Ehrlich and others-Ehrlich's "side chain theory." In a popular treatise a simple statement of well ascertained facts will, I hope, be appreciated, while an exposition of theories still under discussion might prove wearisome.

It has been shown that the antitoxins when mixed with toxins in a test-tube exhibit their specific neutralising action as shown by the innocuousness of the mixture when injected beneath the skin of a susceptible animal.

The antitoxin of snake poison, which has been successfully used in India for the cure of persons bitten by the deadly cobra, when mixed with cobra venom in proper proportion completely neutralises the poisonous properties of this venom. Such a mixture injected beneath the skin of a small animal is without effect. But if the mixture is heated to $70^{\circ} \mathrm{C}$. the antitoxin is destroyed and by inoculation experiments the toxin is found to be still present and active.

The facts stated show that in certain infectious diseases acquired immunity depends upon the formation of antitoxins in the bodies of immune animals. But these antitoxins have no power to destroy specific disease germs. They neutralise the toxic products of these germs without exhibiting any germicidal action upon the germs themselves. As, 
however, the power of the germs to overcome the resources of nature and invade the blood or tissues depends upon the toxic products developed by them, they are deprived of their power to multiply in the bodies of living animals when these poisonous substances are neutralised. Practically they become as harmless as the common "saprophytic bacteria" which surround us on all sides, and are swallowed in countless numbers with every glass of unsterilised water we drink.

But there is another class of substances, developed during certain diseases, which exhibit specific germicidal activity and have no antitoxic value. Such substances are found in the blood of animals which have been made immune to the pathogenic action of the cholera spirillum, the typhoid bacillus, and the bacillus of hog cholera.

The writer, some ten years ago, obtained experimental evidence which indicates that smallpox immunity probably depends upon a substance which destroys the smallpox germ, rather than upon an antitoxin.

Further details with reference to the antitoxins will be found in Part Second of this volume, in which questions relating to infection, disinfection, and immunity will be discussed in connection with the more important infectious diseases, considered separately. 


\section{PART SECOND}

SPECIFIC INFECTIOUS DISEASES 



\section{CHAPTER I}

\section{BUBONIC PLAGUE}

$\mathrm{HE}$ history of bubonic plague extends back to
a remote antiquity. Greek physicians of the second and third century before the Christian era have left a record of a pestilential malady characterised by the formation of buboes, which prevailed in Libya, in Egypt, and in Syria ; and two Alexandrian physicians, Dioscorides and Poseidonios, who were contemporaries of Christ, have given a description of the disease which leaves no doubt as to its identity with the plague of more recent times. It may be well to explain at this point that the buboes characteristic of the disease are enlarged and inflamed glands in the groins, in the armpits, and elsewhere, which in chronic cases may suppurate and discharge a virulent pus by which the disease is propagated. We now know that the germ of the disease is found not only in these suppurating buboes but also in the blood of an infected individual. 
Three forms of the disease are recognised by modern authors-one a mild or abortive form, in which there is little pain or fever and in which the buboes rarely suppurate. In this form the enlarged glands in the groin, armpit, and neck usually disappear in about two weeks. In its usual form the disease is ushered in with chilly sensations, fever, lassitude, and pain in the back and limbs. The buboes are quickly developed and the general symptoms soon assume a grave character. If the patient lives for a week or more, the buboes usually suppurate and carbuncles and boils are often developed. In the third or fulminant form of the disease, death may occur within a few hours from the outset of the attack and in advance of the development of the characteristic buboes. These cases could scarcely be recognised were it not for the fact they occur during the epidemic prevalence of the disease among persons who have been exposed to infection.

From the first to the sixth centuries of the Christian era we have no authentic accounts of the prevalence of bubonic plague, but there is no reason to believe that it had entirely disappeared from those countries in which it had previously prevailed. During the sixth century, however, its ravages were greatly extended and it prevailed as a devastating epidemic in many parts of the Roman Empire, both of the East 
and of the West; indeed, in the time of Justinian it extended far beyond the limits of the Roman Empire. The origin of this extensive epidemic which raged for more than half a century appears to have been in Lower Egypt in the year 542; thence it extended in one direction along the north coast of Africa, and in the other into Palestine and Syria. The following year it invaded Europe, which at the time was in a state of political disturbance and warfare, and during this and subsequent years it devastated many sections of the country, depopulating towns and leaving the country in some instances nothing more than a desert inhabited by wild beasts. The accounts given of this widespread epidemic indicate that other infectious maladies, which at the time had not been clearly recognised as specific diseases, were associated with the plague and contributed to the general mortality.

During the middle ages epidemics continued to occur, but the accounts of the nature of the prevailing "pest" are usually confused and unsatisfactory, and it was not until nearly the middle of the fourteenth century that the horrible epidemic known as the black death devastated Europe and caused the death of more than $25,000,000$ of its inhabitants. There has been considerable difference of opinion among the best authorities as to whether the black 
death of the fourteenth century was identical with bubonic plague. It presented some features which seem to distinguish it from subsequent epidemics, and it had its origin from a different quarter of the globe. While bubonic plague has usually invaded Europe from Egypt, the black death is believed to have originated in Northern China. It is not known exactly when or where this epidemic had its origin, but it is known to have reached the Crimea in 1346 and Constantinople the following year. The same year it was conveyed by ships to several seaports of Italy both on the Mediterranean and the Adriatic, and also to Marseilles on the French coast; in 1348 it extended to the interior of these countries and to Spain; also to England, Holland, and the Scandinavian peninsula. The following year it completed the invasion of Europe.

The disease first appeared in London in November, 1348 , and it continued to prevail in various parts of England for a period of eight or nine years. In $135^{2}$ the epidemic prevailed in the town of Oxford to such an extent that this town lost two-thirds of its academic population. The plague again invaded England in 1361 and 1368 . As a result of these devastating epidemics in England, as well as in other parts of Europe, large parts of the country remained for a time uncultivated, and owing to 
the lack of labourers there was a great increase in wages.

The following graphic account of the ravages of this pestilence is by a writer of the period:

"Wild places were sought for shelter; some went into ships and anchored themselves far off on the waters. But the angel that was pouring the vial had a foot on the sea as well as on the dry land. No place was so wild that the plague did not visit none so secret that the quick-sighted pestilence did not discover -none could fly that it did not overtake. For a time all commerce was in coffins and shrouds, but even that ended. Shrift there was none; churches and chapels were open, but neither priests nor penitents entered-all went to the charnel-house. The sexton and the physician were cast into the same deep and wide grave; the testator and his heirs and executors were hurled from the same cart to the same hole together. Fire became extinguished, as if its element had expired, and the seans of the sailorless ships yawned to the sun. Though doors were open and coffers unwatched, there was no theft; all offences ceased, and no cry but the universal woe of the pestilence was heard among men."

That the "black death" of the fourteenth century was in fact the same disease which subsequently prevailed in Europe under the name of "the plague," and more recently known as "bubonic plague," can scarcely be doubted. But the epidemic was characterised by an unusually large number of cases of the pulmonary form of the disease, in which it seems probable that the lungs are the primary seat of infection, while in the bubonic form the bacillus effects a 
lodgment through some superficial wound or abrasion, or possibly through the bites of insects, and it first invades the lymphatics, producing inflammation of the nearest lymphatic glands. General invasion of the blood appears, from recent investigations, to be a secondary phenomenon which only occurs in very severe and usually fatal cases.

The pulmonic form of the disease, which was so prominent in the epidemic known as black death, is extremely fatal and is known to occur at the present day.

Bubonic plague continued to prevail in various parts of Europe at the end of the sixteenth century, and early in the seventeenth century (1603) an epidemic occurred in London which caused the death of 38,000 of its inhabitants. It continued to prevail in this city and in various parts of England, Holland, and Germany, and six years later caused a mortality of 11,785 in the city of London. During the year 1603 a most disastrous epidemic occurred in Egypt, which is said to have caused a mortality of at least a million. After an interval of ten or fifteen years, during which there was a marked diminution in the number of cases and the extent of its distribution in European countries, it again obtained wide prevalence during the year I620 and subsequently, especially in Germany, Holland, and England. The epidemic in the city of 
London in 1625 caused a mortality of more than 35,000 . In 1630 a severe epidemic occurred in Milan, and in 1636 London again suffered a mortality of over 10,000, while the disease continued to claim numerous victims in other parts of England and on "the continent." Later in the century ( 1656 ) some of the Italian cities suffered devastating epidemics. The mortality in the city of Naples was in the neighbourhood of 300,000 , in Genoa 60,000, in Rome 14,000. The smaller mortality in the lastnamed city has been ascribed to the sanitary measures instituted by Cardinal Gastaldi. Up to this time prayers, processionals, the firing of cannons, etc., had been the chief reliance for the arrest of pestilence, with what success is shown by the brief historical review thus far presented. This enlightened prelate inaugurated a method of combating the plague and other infectious maladies which, with increasing knowledge and experience in the use of scientific preventive measures, has given us the mastery of these pestilential diseases, and has been the principal factor in the extinction of bubonic plague from the civilised countries of Europe.

But it was long after the time of Cardinal Gastaldi before sanitary science was established upon a scientific basis and had acquired the confidence of the educated classes. Indeed the golden age of 
preventive medicine has but recently had its dawn, and sanitarians at the present day often encounter great difficulty in convincing legislators and the public generally of the importance of the measures which have been proved to be adequate, when properly carried out, for the prevention of this and other infectious maladies.

We have now arrived in our review at the period of the "great plague of London." For some years this city had been almost if not entirely free from the scourge, but in the spring of 1665 it again appeared and within a few months caused a mortality of 68,596 in a population estimated at 460,000 . This, however, does not fairly represent the percentage of mortality among those exposed, for a large proportion of the population fled from the city to escape infection.

Upon the continent the disease prevailed extensively, especially in Austria, Hungary, and Germany. The epidemic in Vienna in 1679 caused a mortality of 76,000. In I68 I the city of Prague lost 83,000 of its inhabitants. During the last quarter of this century the disease disappeared from some of the principal countries of Europe. According to Hirsch, it disappeared from England in 1679, from France in 1668, from Holland about the same time, from Germany in 1683, and from Spain in 1681. In Italy it 
continued to prevail to some extent until the end of the century.

At the beginning of the eighteenth century bubonic plague prevailed in Constantinople and at various points along the Danube; from here it extended in I 704 to Poland, and soon after to Silesia, Lithuania, Germany, and the Scandinavian countries. The mortality in Stockholm was about 40,000. The disease also extended westward from Constantinople through Austria and Bohemia.

In I 720 Marseilles suffered a severe epidemic, probably as a result of the introduction of cases on a ship from Leghorn. The mortality was estimated as being between 40,000 and 60,000. From Marseilles as a centre it spread through the province of Provence, but did not invade other parts of France. In I 743 a severe outbreak occurred on the island of Sicily. A destructive but brief epidemic, which is estimated to have caused a mortality of 300,000 , occurred during the years I770 and I77I in Moldavia, Wallachia, Transylvania, Hungary, and Poland. At the same time the disease prevailed in Russia, and in $177 \mathrm{I}$ caused the death of about one-fourth of the population of the city of Moscow.

Early in the nineteenth century (1802) bubonic plague appeared at Constantinople and in Armenia. It had previously prevailed in the Caucasus, from 
which province it extended into Russia. In I 808 to 18 I 3 it extended from Constantinople to Odessa, to Smyrna, and to various localities in Transylvania. It also prevailed about the same time in Bosnia and Dalmatia. In 1812 to 1814 it prevailed in Egypt, and, as usual, was conveyed from there to European countries. During the same year it prevailed extensively in Moldavia, Wallachia, and Bessarabia. In 1831 it again prevailed as an epidemic in Constantinople and various parts of Roumelia, and again it appeared in Dalmatia in 1840 and in Constantinople in 1841. Egypt, which for centuries had been the principal focus from which plague had been introduced into Europe, continued to suffer from the disease until 1845, when it disappeared from that country.

The last appearance of Oriental plague in Europe, until its recent introduction into Portugal, was the outbreak on the banks of the Volga in 1878-79. The disease had previously prevailed in a mild form in the vicinity of Astrakhan and was probably introduced from that locality. An interesting fact in connection with this epidemic is that in Astrakhan the disease was so mild that no deaths occurred, and that the earlier cases on the right bank of the Volga were of the same mild form, but that the disease there increased rapidly in severity, and soon became so ma- 
lignant that scarcely any of those attacked recovered. This is to some extent the history of epidemics elsewhere, and not only of plague, but of other infectious diseases, such as typhus fever, cholera, and yellow fever. In all of these diseases the outset of an epidemic may be characterised by cases so mild in character that they are not recognised, and during the progress of the epidemic many such cases may continue to occur. These cases are evidently especially dangerous as regards the propagation of the disease, for when they are not recognised no restrictions are placed upon the infected individuals, although they may be sowing the germs broadcast.

The termination of an epidemic in the pre-sanitary period depended to a considerable extent upon the fact that those who suffered a mild attack acquired thereby an immunity, and that when the more susceptible individuals in a community had succumbed to the prevailing disease there was a necessary termination of the epidemic for want of material.

Another factor which, no doubt, has an important bearing upon the termination of epidemics is a change in the virulence of the germ as a result of various natural agencies. Time will not permit me to discuss this subject in its scientific and practical aspects, but the general fact may be stated that all known disease germs may vary greatly in their pathogenic 
virulence, and that in every infectious disease mild cases may occur, not only because of the slight susceptibility of the individual, but also because of the "attenuated" virulence of the specific germ. In the eighteenth century, the beginning of sanitary science, isolation of the sick, and seaboard quarantines came to the aid of these natural agencies, and did much in the way of arresting the progress of this pestilential disease. At the present day these measures, together with disinfection by heat or chemical agents, are relied upon by sanitarians with great confidence as being entirely adequate for the exclusion of this disease or for stamping it out if it should effect a lodgment in localities where an enlightened public sentiment permits the thorough execution of these preventive measures; but when the disease prevails among an ignorant population which strenuously objects to the carrying out of these measures, the contest between the sanitary officer and the deadly germ is an unequal one, and the stamping out of an epidemic becomes a task of great magnitude, if not entirely hopeless. This is illustrated by the experience of the English in their encounter with bubonic plague in their Indian Empire.

Plague seemed to be almost a thing of the past and no longer gave any uneasiness in the countries of Europe which had formerly suffered from its rav- 
ages, when in February, I894, it made its appearance in the city of Canton, China, and three months later in Hong Kong. The disease is known to have been epidemic in the province of Yunnan, which is about nine hundred miles distant from Canton, since the year 1873 , but it attracted little atten-

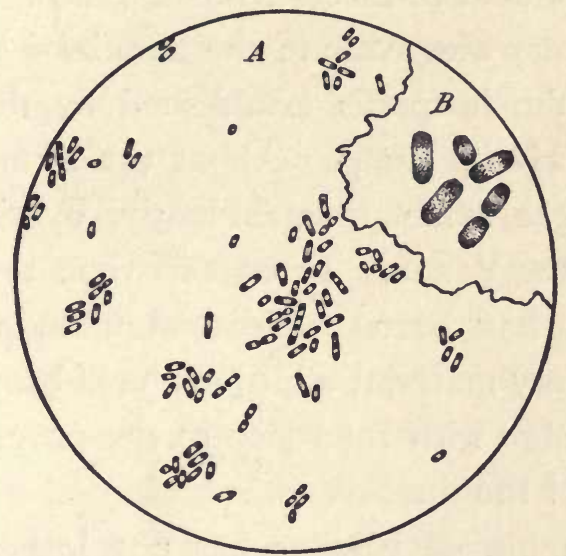

FIG. I. Bacillus of bubonic plague. $A$, magnified rooo diameters; $B$, more highly magnified to show " end-staining."

tion until the lives of Europeans living in the city of Hong Kong were threatened by the outbreak of an epidemic among the Chinese residents of that place. Many thousands of deaths occurred in Canton during the three months which elapsed after its introduction to that city before it effected a lodgment in Hong Kong.

Fortunately this outbreak gave the opportunity 
for competent bacteriologists to make scientific investigations relating to the specific cause of this scourge of the human race and to the demonstration that it is due to a minute bacillus. This discovery was first made by the Japanese bacteriologist, Kitasato, who had received his training in the laboratory of the famous Professor Robert Koch, of Berlin. This discovery was made in the month of June, I 894 , in one of the hospitals established by the English officials in Hong Kong. About the same time the discovery was made, independently, by the French bacteriologist, Yersin. From this time the study of the plague has been established upon a scientific basis and very material additions have been made to our knowledge with reference to the prevention and treatment of the disease.

That the plague bacillus has not lost any of its original virulence is amply demonstrated by the high death-rate among those attacked, and we are justified in ascribing its restricted prevalence to the general improvement in sanitary conditions in civilised countries and to the well directed efforts of public health officers in the various localities to which it has been introduced during recent years. In the Philippine Islands, where it prevailed to a considerable extent when our troops first took possession of the city of Manila and where the conditions among the natives 
are extremely favourable for its extension, it has been kept within reasonable bounds and, indeed, the latest reports indicate that it has been practically exterminated by the persistent efforts of the medical officers of our army, charged with the duty of protecting the public health in those islands.

The monthly report of the Board of Health for the city of Manila for September, 1902, the last at hand, records but one death from plague during that month. During the same period there were ten deaths from typhoid fever, thirty-five deaths from dysentery, and seventy-six deaths from "the great white plague," pulmonary tuberculosis.

Bubonic plague, cholera, and typhoid fever have long been classed as "filth-diseases," and in a certain sense this is correct, although we now know that the germs of these diseases not only are not generated by filth, but do not multiply in accumulations of filth. They are present, however, in the alvine discharges of the sick, and when this kind of filth is exposed in the vicinity of human habitations or gains access to wells or streams, the water of which is used for drinking, the germs are likely to be conveyed to the alimentary canals of susceptible individuals, and thus the disease is propagated. Until quite recently the attention of sanitarians was so firmly fixed upon the demonstrated transmission of cholera and typhoid 
fever through the agency of contaminated water or milk that certain other modes of transmission were overlooked, or at least underrated. I refer to the transmission by insects, or as dust by currents of air. I have for many years insisted upon the part played by flies as carriers of infectious material from moist masses of excreta from cases of cholera and typhoid fever. There is good reason to believe that the bacillus of bubonic plague may be transmitted in the same way.

Certain of the lower animals, including rats and mice, are very susceptible to infection, and they play an important part in the propagation of the disease. The germs are found not only in the blood and in pus from suppurating buboes, but also in the discharges from the bowels of the sick and of infected rats. This being the case it can readily be seen how important a strict sanitary police is in arresting the spread of an epidemic.

Dr. James A. Lowson, who has written an excellent account of the epidemic in Hong Kong, says :

"Filth and overcrowding must be recorded as two of the most important factors. The district of Torpingshan supplied these factors in a marked degree at the beginning of the outbreak, the majority of the houses being in a most filthy condition, as, owing to the uncleanly habits of the people, the amount of what is generally termed rubbish accumulates in a Chinese house in a crowded city to an extent beyond the imagi- 
nation of civilised people. When to a mixture of dust, old rags, ashes, broken crockery, moist surface soil, etc., are added fecal matter and the decomposing urine of animals and human beings, a terribly insanitary condition of affairs prevails."

The period of incubation in bubonic plague-the time which elapses between exposure to infection and the development of the disease-is usually from three to six days.

From the report of Dr. Lowson of cases treated in the various hospitals of Hong Kong, under the control of English physicians, it appears that the mortality was much greater among natives of Hong Kong than among the foreign residents of that city. The mortality among Europeans (I I cases only) was I 3.2\%; among Japanese (Io cases), $60 \%$; among Portuguese ( 18 cases), $66 \%$; among Chinese (2619 cases), $93.4 \%$. To a considerable extent, no doubt, this difference in mortality was due to the unfavourable surroundings of the natives and their lack of proper nursing and medical attendance, many of them being brought to the hospital in a dying condition.

Experiments upon rats and other animals show that they become infected when cultures of the plague-bacillus are deposited upon the mucous membrane of the nose.

During the epidemic prevalence of the disease these animals die in large numbers, and there is good 
reason to believe that they play an important part in the propagation of the malady. It has been suggested that infection may be carried from rats to man through the agency of fleas which swarm upon these rodents and desert them when they die.

The Japanese physician, Aoyoma, who was associated with Kitasato, and who contracted the disease but recovered, is of the opinion that in a great majority of the cases, and perhaps in all, infection occurs through an external wound. He calls attention to the fact that physicians and nurses in attendance upon cases of the disease rarely become infected, and states that during the epidemic of 1894 in Hong Kong only three Japanese and one Chinese physician became infected, while all of the nurses escaped; also to the fact that of three hundred English soldiers who volunteered to clean and disinfect the Chinese pesthouses during the prevalence of the epidemic, only ten contracted the disease. The greater liability of the lower class of natives to contract the disease, he ascribes not only to the insanitary surroundings in which they live, but also to the fact that they seldom wear shoes and stockings, and thus are very liable to infection through insignificant wounds, scratches, or abrasions, both of the feet and hands. In this connection it is well to call attention to the fact that in former epidemics physicians have suffered severely, 
and that whatever immunity they enjoy is due to the observance of sanitary precautions, the importance of which has become apparent as we have acquired a more exact knowledge of the etiology of the disease.

During the past few years a number of prominent bacteriologists have been engaged in researches relating to the prevention and cure of bubonic plague by means of an antitoxic serum, obtained by the same method and in accordance with the same fundamental scientific principle as in the case of the antitoxic serum which is now so successfully employed in the treatment of diphtheria. The experiments thus far made have apparently been attended with a considerable degree of success. Professor Calmette reports that the serum of Yersin prepared at the Pasteur Institute in Paris proved to be curative in a considerable proportion of the cases treated during the recent outbreak at Oporto, and that protective inoculation conferred a temporary immunity, which, however, did not last longer than twenty days. The mortality in cases not treated by Yersin's serum was $70 \%$, in those treated with it $13 \%$.

The inoculations made by Haffkine in Bombay appear to have been quite successful. In his first experiment 8142 persons were inoculated. Of these, I 8 subsequently contracted the disease and 2 died. Among 4926 persons inoculated a single time at 
Dharwan 45 were subsequently attacked and 15 died; while among 3387 persons in whom a second inoculation was made, only 2 were attacked. Haffkine uses in his inoculations a sterilised culture of the plague-bacillus. The inoculation is followed by slight fever and enlargement of the nearest lymphatic glands. All symptoms disappear at the end of two or three days.

The plague-bacillus is very easily destroyed by disinfectants. Dr. Lowson reports that a I \% solution of carbolic acid kills the bacilli within an hour, a $2 \%$ solution almost immediately. Quicklime was almost as prompt in its action. Exposure to fresh air for three or four days usually destroys the vitality of the bacillus, and exposure to direct sunlight destroys it in three or four hours. Kitasato and Yersin both arrived at the conclusion that the disease may be contracted by inoculation, through a wound or abrasion; by way of the respiratory tract when the bacillus is present in dust carried by the inspired air; or by way of the stomach when food or drink taken contains the bacillus.

What has been said indicates very clearly the proper preventive measures, and when these are enforced with energy and intelligence there need be little fear of the epidemic extension of the disease.

For the prevention of this and other filth diseases, 
our main reliance must rest upon isolation of the sick, disinfection of all infectious material, and general sanitary police of infected localities. The destruction of rats in localities infected, or likely to become so, is also a measure of prime importance. Protective inoculations will hardly be necessary if the measures above referred to are vigorously enforced, and in the opinion of the writer it is only under exceptional circumstances that such inoculations need be practised on a large scale. Those whose duties require them to care for the sick or visit infected localities may be given such protection as is afforded by Haffkine's inoculations. But the immunisation of entire communities by inoculation in anticipation of a possible plague epidemic hardly seems necessary in the present state of sanitary knowledge. 


\section{CHAPTER II}

\section{ASIATIC CHOLERA}

A SIATIC cholera has its permanent home in India, and especially in the thickly populated region occupied by the delta of the Ganges, the principal city of which is Calcutta. Here it prevails throughout the year, with a period of maximum intensity in the hot and comparatively dry month of April. Heavy rains have a salutary sanitary influence in this and other regions where the disease is prevalent. From the infected area referred to, the disease spreads almost annually to other parts of India and neighbouring Asiatic countries, and at intervals has extended its ravages to Africa, Europe, and America. These widespread epidemics are, however, of comparatively recent origin.

After a devastating epidemic in India during the years 1816 to 1819 the disease extended to Mauritius and the east coast of Africa, to Farther India and the islands of Sumatra, Java, and Borneo, and to China. 
Two or three years later the disease prevailed in Syria and Palestine, and for the first time, so far as is known, appeared in a European country, in Astrakan. A second great epidemic, beginning in India in 1826 , reached Astrakan in 1830 . Thence it extended as far north as St. Petersburg. The following spring it invaded Poland and extended from Austria into Germany. At the same time it had been carried by way of Persia, Mesopotamia, Arabia, and Palestine into Egypt. Hungary, Austria, and Turkey were invaded during the same year (1831), and it finally reached Great Britain, where in 1832 it obtained wide prevalence. In this year also France, Belgium, and Holland were invaded.

In 1832 it reached the United States by way of Canada, where it was carried by Irish immigrants. It spread rapidly along the St. Lawrence and the shores of Lake Ontario. By an independent importation it was brought in the same year to New York and spread thence southward and westward, invading the States of Pennsylvania, Virginia, Maryland, Kentucky, Ohio, Indiana, and Illinois, and in October first appeared in the city of New Orleans. The following summer it broke out afresh in this city and rapidly spread through the southern, central, and western States. It also invaded the Indian Territory and California. In 1834 it reappeared in the eastern 
States, and in 1835 was reintroduced from Cuba to the city of New Orleans. In the meantime Mexico had been invaded, and subsequently the disease obtained a limited prevalence in Central America and the Gulf coast of South America. Simultaneously with this widespread epidemic in America the disease spread in Europe to Sweden and Norway in the north and to Italy, Spain, and Portugal in the south. The disease died out during the winter of $1837-38$, and for the ten following years Europe, Africa, and America remained free from this scourge of the human race. In 1846 the disease, which had previously shown increased epidemic extension in India, obtained wide prevalence in Persia, thence it extended to Arabia, and the following year reached Constantinople. In I 848 it spread through Turkey, Hungary, Asia Minor, Syria, and Egypt.

In the meantime it had been introduced into Russia (in I 847), and early in the summer of 1848 reached Germany. In the autumn of this year it was introduced into England and Scotland, and the following year obtained wide prevalence in the British Isles. Holland and Belgium were invaded at the same time, and in I 849 France and Austria suffered severe epidemics. Northern Italy also suffered from the disease, which indeed prevailed in all parts of Europe, with the exception of Spain and Portugal, which countries 
remained exempt. In December, 1848, cholera was brought from Europe, by emigrant ships, both to New York and to New Orleans. From the lastnamed city it spread northward to Memphis and westward into the State of Texas, and during the year I 849 nearly all of the States east of the Rocky Mountains suffered to a greater or less extent, especially those located in the valleys of the Ohio and Mississippi rivers. It continued to prevail during the following year ( 1850 ), and in October was carried to San Francisco by way of the Isthmus of Panama. During this epidemic the West Indies suffered severely, especially the islands of Cuba and Jamaica. It also prevailed in Mexico, and to some extent in South America. The epidemic did not terminate in the Western Hemisphere until the end of the year I 854, but in Europe it had practically ceased to prevail, except in a few localities in northern Europe, by the close of the year I 850 .

In the spring of 1852 another widespread epidemic was inaugurated. The disease appeared almost at the same time in Persia, Mesopotamia, and Poland, apparently as a survival from the previous epidemic, and extended to adjacent countries. In Russia it continued to prevail to some extent during a period of ten years, the years 1853, 1855, and 1859 being notable as marking its widest extension and the 
greatest mortality. This was also the case in northern Germany. In 1855, Austria and Italy suffered severely. The disease was brought to England from Germany in 1853 and extended to various parts of the island during this and the following year. France also suffered severely from the disease at this time (1853-54), and also Spain and Portugal, where it prevailed during the period from 1853 to 1860 . In North America the disease was introduced into Canada in 1853 , and obtained wide extension throughout the United States in 1854. The disease disappeared from the countries of North America the following year, but in Central America and in South America it prevailed to some extent for several years subsequent to this date, although the year 1855 was that of greatest extension and fatality.

Another epidemic period is that embraced between the years 1865 and 1875 . As usual, the epidemic extension of the disease in Europe was preceded by a period of increased fatality in its native haunts, in the lower basin of the Ganges, and also by its extension to other eastern countries - China, Japan. I shall not attempt to follow the progress of the disease through the countries of Europe, but, as showing its fatal character, will give some mortality statistics. The epidemic of 1867 in Italy was attended by a mortality of 130,000 . In Prussia, during 
the year I 866 , I 14,683 deaths were reported. In England the disease, in 1865 and the next year, caused a mortality of 14,378 , nearly half of the deaths occurring in the city of London. In Belgium a mortality of 32,812 occurred during the year of greatest prevalence (i 866).

In North America the disease was introduced in I 866 by three independent importations, to New York, New Orleans, and Halifax. The following year it obtained wide extension, especially in the western States and in Texas.

Again cholera invaded Europe during i $87 \mathrm{I}-73$, and again it was introduced into the United States by way of New Orleans, and thence spread to some extent in the valley of the Mississippi, and along its principal tributaries. The epidemic of 1872 is estimated to have cost 120,000 lives in Russia, and the previous year the mortality was still greater. In Hungary the disease claimed 190,000 victims during the years 1872 and 1873 . In Prussia the number of deaths in 1873 was 28,790 .

Cholera disappeared from Europe and America in I873, and the next great epidemic was inaugurated in 1884 , when it reached the shores of France and Italy. According to Dr. Shakespeare, ${ }^{1}$ this epidemic " cost France 15,000 of its inhabitants in I884, I 885,

${ }^{1}$ Report on Cholera in Europe and India, by E. O. Shakespeare, M.D. (1890). 
and I886; Spain, I80,000 in 1884 and 1885 ; Austria-Hungary, 4000 inhabitants in 1886; Italy, about 50,000 inhabitants in 1884,1886 , and I $887 . "$

In Japan, the epidemic of 1885 was attended with a mortality of 109,434 .

In 1891 cholera prevailed extensively in India, Syria, Arabia, Siam, and Japan, and was introduced into Austria by way of Persia. By November, 1892, it is estimated that half a million cases had occurred in Russia with a mortality of at least 50 per cent. A severe outbreak of the disease occurred in the city of Hamburg during the month of August of this year, resulting in 8005 deaths. From Hamburg the disease was brought to New York harbour on several ships carrying immigrants, but owing to the vigorous measures adopted by the local health authorities, assisted by the Marine Hospital Service, the disease was arrested at the threshold of the country and an epidemic was averted. The success obtained at this time shows what can be accomplished by sanitary measures based upon an exact knowledge with reference to the specific cause of the disease (cholera germ) and its mode of transmission. The extension of cholera from its home in India to the countries of Europe and America, which first occurred during the nineteenth century, was no doubt due to the increased facilities for rapid transit, especially by steamboats 
and railroads. Infected individuals leaving the localities where they had contracted the disease, would fall by the way, or recover before travelling any great distance, if restricted to the methods of transportation available before the introduction of steam as a motive power for ships and railroad coaches. The fact that cholera is carried from place to place by men, following routes of travel, is well stated by the German physician Griesinger. He says :

"Cholera has never advanced like a broad stream inundating entire countries at one time, bringing disease to all regions lying parallel with each other, over a wide extent, but it always advances in relatively narrow lines from which usually, but not always, lateral offshoots arise. In countries with a small population we see constantly that this district corresponds with the great lines of travel. If the disease oversteps high mountains, if it passes through a desert, if it crosses the ocean, it always follows the lines of human intercourse, the post and military routes, the caravan and sailing routes, etc. If it has broken out on an island, then the first cases have always been in a seaport, never in the interior."

We now know definitely that the cholera germ is carried from place to place by cholera-infected individuals, who harbour this deadly spirillum in their intestines and leave it wherever the discharges from their bowels are deposited. Mild cases of "choleraic diarrhœa" are even more dangerous as regards the propagation of the disease than severe and fatal cases, for the infected individual may not suspect the nature 
of a malady which he considers trivial, and, instead of being isolated and properly treated as he should be in his own interest and that of the public generally, he goes from town to town or from country to country distributing cholera germs by the way.

Cholera is contracted only by the introduction of cholera germs into the intestinal canal by way of the mouth and stomach. The spirillum is easily destroyed by acids, and consequently does not multiply in the healthy human stomach, and, under ordinary conditions, probably does not survive to reach the intestine, owing to the germicidal action of the acid gastric juice. But any derangement of the digestive process predisposes to an attack of the disease. Excesses in eating or drinking cause the individual to be more susceptible to infection. In Germany it has been noted that an increase in the number of cases is likely to occur on Sunday, because of the indulgence of the labouring classes in wine and beer during this day of rest and recreation.

Invalids, and especially those subject to disorders of the stomach and bowels, are especially liable to contract the disease during its epidemic prevalence. But it is safe to say that no one will contract cholera unless living cholera germs are introduced into the intestinal canal. This usually occurs as a result of drinking cholera-infected water, or partaking of food 
which in some way has become infected and which has not been sterilised by heat. The cholera spirillum is so easily destroyed by heat that the prevention of the disease is a comparatively simple matter. Any article of food or drink which has been subjected for a few minutes to a temperature of $140^{\circ}$ Fahr. $\left(60^{\circ}\right.$ C.) is absolutely safe, unless it has been reinfected after such exposure. Such reinfection might occur if the sterilised food or liquid is exposed to contamination by infected flies. These insects, without doubt, not infrequently act as carriers of infection from exposed cholera excreta-on the ground or in shallow pits-to articles of food or drink which they light upon or fall into. When the water supply of a town becomes infected as a result of contamination by cholera excreta, a general epidemic is almost sure to occur, unless those using the water are sufficiently intelligent and well informed to take the precaution of sterilising, by heat, all water used for drinking purposes. When this is done there is little liability to infection through other channels, with the exception of the milk supply-which should also be sterilised by heat-and the danger from flies already referred to. This latter source of infection is to be guarded against by a strict sanitary police, by which all exposed excreta are covered with earth or disinfected, and by the use of fly screens and other 
methods of getting rid of these dangerous domestic pests. But the most important sanitary precaution consists in the immediate disinfection of all cholera excreta. This should be effected so far as possible in the sick-room or its immediate vicinity. And the dangerous mild cases of choleraic diarrhœea should be looked for and placed under treatment, an important part of which will be complete rest and isolation under conditions which admit of the necessary measures of disinfection.

A channel of infection not yet mentioned, is through the soiled underclothing or bed linen of cholera patients. Laundresses have not infrequently contracted the disease as a result of handling such infected articles. For this reason disinfection by immersion in boiling water or a suitable disinfecting solution should be carried out in the sick-room, or an adjoining apartment.

The cholera germ, which was discovered by the German bacteriologist, Dr. Robert Koch, in 1884 , is usually seen in the form of slightly curved rods, resembling somewhat a comma, and was first spoken of as "the comma bacillus of Koch." But these slightly curved rods may grow out into longer or shorter spiral filaments, and the so-called comma bacillus is described in systematic works upon bacteriology as a "spirillum" (Spivillum cholerce Asiatica). It is con- 
stantly found in the excreta of persons suffering from cholera, in the mild cases of choleraic diarrhœa as well as in the severe and fatal form of the disease. Fortunately for the human race, this cholera spirillum is a tender exotic which has not succeeded in establishing itself permanently in Europe and America. Although not killed by cold, its epidemic extension is

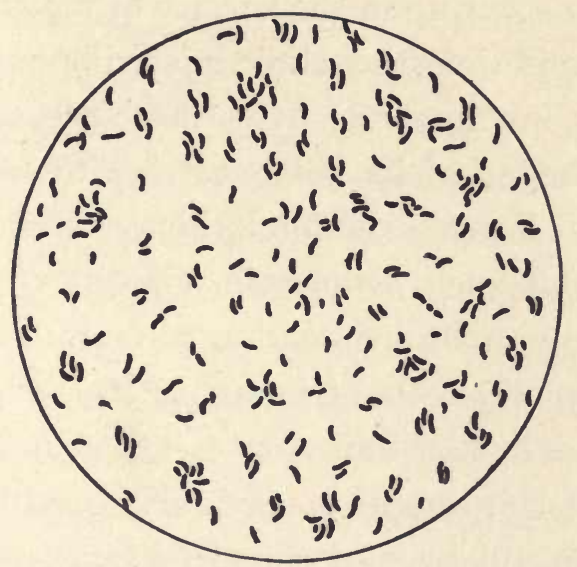

FIG. 2. Spirillum of Asiatic cholera (" comma bacillus of Koch "); magnified sooo diameters.

arrested upon the approach of winter; and our brief historical review of its prevalence, outside of its home in India, shows that after a period of longer or shorter duration the disease dies out and does not occur again independently of a fresh importation. Under favourable conditions as to temperature, the cholera spirillum multiplies in well or river water, 
especially if this contains a certain amount of organic impurities. It has been shown by experiment that it will multiply abundantly in distilled water to which from two to three per cent. of bouillon has been added, also that it may preserve its vitality in sterilised well or river water for several months. Koch found in his early investigations that rapid multiplication may occur upon the surface of moist linen. In a moist condition the cholera spirillum may retain its vitality for months. It is therefore evident that there is danger of its being carried to remote localities in bed linen or underclothing soiled by cholera discharges, if such articles in a moist condition are packed up, without disinfecting, to be transported with other personal effects of immigrants. Exposure to a temperature of $140^{\circ} \mathrm{Fahr} .\left(60^{\circ} \mathrm{C}\right.$.) destroys the cholera spirillum very promptly. It is also quickly killed by desiccation and by exposure to direct sunlight - two to four hours' exposure. Experiments made in Dr. Koch's laboratory show that it is killed in two hours by sulphuric acid or hydrochloric acid diluted in the proportion of one part to 1300 parts of water; by carbolic acid I: 400 ; by lysol I: 500 ; by corrosive sublimate $1: 10,000$; by sulphate of copper $1: 500$. Solutions of this strength would disinfect soiled linen, but in practice it will be best to use the agents mentioned in much stronger solutions-one-per-cent. solutions 
of carbolic acid, lysol, or sulphate of copper, or one part of corrosive sublimate to 1000 of water. For the disinfection of cholera excreta, milk of lime is to be recommended (see page 59), or a five-per-cent. solution of carbolic acid or lysol, or a two-per-cent. solution of chloride of lime, or boiling water.

Sanitarians no longer have any great apprehension with reference to the extension of this Asiatic plague in European countries or in America. The measures for its prevention are simple and easily applied, and it is only in localities where ignorance or prejudice stands in the way of the execution of these means that the deadly spirillum is likely to establish itself in civilised countries. The measures referred to may be summed up briefly as follows: Isolation of the sick, including all cases of choleraic diarrhœa; disinfection of excreta; sanitary police of infected localities; boiling of drinking water; exclusion of flies from dwellings, and especially from kitchens.

The last-mentioned sanitary precaution is perhaps the most difficult of execution. But, fortunately, it is not essential when the measures previously mentioned have been carried out, especially the disinfection of excreta. Indeed all other measures might be neglected as superfluous if we could be assured of the complete destruction of all cholera germs in the excreta of infected individuals. And if this could be 
accomplished in the countries where the disease has its permanent habitat, there is every reason to believe that its complete extinction would soon be effected.

The prevention of cholera by "protective inoculations" has been tested on a large scale in Spain, during the epidemic of 1885 , and more recently in India, by the method of Haffkine.

Unfortunately the evidence relating to the value of these protective inoculations is not very satisfactory. The evidence, however, is in favour of the view that a partial and temporary immunity may be conferred by the subcutaneous injection of cultures of the cholera germ. We should not expect such inoculations to confer an absolute immunity, inasmuch as this does not result from an attack of the disease. That repeated attacks may occur in the same individual is well established, and this may happen during a single epidemic, as was observed in the last Hamburg epidemic.

That the inoculated are not exempt from attack is shown by Haffkine's statistics with reference to inoculations made by him in India (1895). Five hundred inoculated individuals gave a mortality of 19 , or 3.8 per cent., while among 1735 non-inoculated, under similar conditions, the mortality was 6.5 per cent. In our opinion inoculation as a method of prevention should not be employed on a large scale in anticipa- 
tion of an epidemic, as it is far less reliable than the preventive measures heretofore referred to-disinfection of excreta, boiling of drinking water, etc. But for individuals who are required, for any reason, to remain in an infected locality in India or elsewhere, it is perhaps worth while to further test the value of this method of prevention. 


\section{CHAPTER III}

TYPHOID FEVER

$\mathrm{B}^{\mathrm{UBONIC}}$ plague, Asiatic cholera, and dysentery are filth diseases which appertain especially to the populous countries of the Orient. In the present chapter we shall consider a filth disease which prevails in all parts of the civilised world, and which continues to claim its annual quota of victims notwithstanding the fact that we know its specific cause (germ), its mode of transmission, and the preventive measures which if thoroughly carried out would soon lead to its extinction. According to the last census return, there were 35,379 deaths from typhoid fever in the United States during the census year 1900. The increase in mortality over the number in $1890(27,056)$ is out of proportion to the increase in population, notwithstanding the general improvement in the sanitary condition of towns and cities. This is no doubt due to the continued pollution of water supplies and to the extension of this infectious disease in rural districts. 
As the typhoid bacillus is contained in the excreta of the sick, no single agency is more important for the prevention of this, and of other filth diseases, than the use of properly constructed sewers for the reception of excreta and its removal from the vicinity of human habitations.

Disease germs contained in human excreta, when this is conveyed, by flushing or otherwise, to properly constructed sewers, are no longer dangerous to the community where these sewers are located. But they may be capable of doing serious harm to other communities if the sewers empty into a stream the water of which is used for drinking purposes.

Sewers had come into use and had the warm endorsement of sanitarians long before the discovery of the germs of the infectious maladies under consideration, and before it was positively known that the infectious agents in these diseases are contained in the discharges from the bowels. But now that we have an exact knowledge of the causes of these diseases, the reason for the beneficent results attending the use of sewers, in connection with an ample and pure water supply, is apparent. It may be safely asserted that a city or town having a complete and satisfactory sewer system and a pure water supply is practically immune from epidemics of cholera or typhoid fever, provided, of course; that the sewers 
are used for the purpose for which they are intended, and that streets and back yards no longer serve as receptacles for filth, as was usual during the presanitary period, even in great cities like London and Paris. The axiom tout a l' égout now governs the practice not only in Paris but wherever the fundamental principles of sanitation are understood and sewers have been constructed.

Unfortunately, the cost of sewer construction, the reluctance of taxpayers to part with their money, and the ignorance or indifference of municipal authorities have conspired to prevent the accomplishment of this fundamental sanitary measure in very many towns in the United States, and our endemic plague - typhoid fever - continues to claim a large annual quota of victims in such localities. Even in the national capital our sewer system is incomplete, and in many out-ofthe-way places, especially in the densely populated alleys of the city, shallow box privies are in use as receptacles for human excreta. The typhoid-fever rate, owing to this and other causes, is disgracefully high.

Mortality rates in towns and cities throughout the civilised world depend to a large extent upon the purity of the water supply and the efficiency of the system of sewage disposal; and the constant improvement which is shown by the mortality statistics 
of England and other countries which have made the most progress in this direction is undoubtedly largely due to these two factors. This is well illustrated by the mortality statistics of armies. In the German army, the annual death-rate in 1868 was 6.9 per thousand, a decade later it was 4.82 , in 1888 it had fallen to 3.24 , and in 1896 to 2.6 . In our own army, the death-rate during the period of peace just prior to the Mexican War (1848) was about three and one-half times as great as during the five years preceding our recent war with Spain; and since the year 1872 there has been a diminution of the death-rate of nearly forty per cent. In the British army at home stations, the mortality rate during the decade ending in 1884 was 7.2 per thousand, in 1889 the rate had fallen to 4.57 , and in 1897 to 3.42 . In the Italian army there has been a gradual and progressive reduction from 13.3 per thousand in 1875 to 4.2 in 1897. The mortality in the French army was a little over 21 per thousand during the five years ending in 1825 . In 1890 it had fallen to 5.8I per thousand.

According to the best estimates, the average of human life in the sixteenth century was somewhat less than twenty years; at the present time it is more than twice as long; and during the past twentyfive years the average duration of life has been 
lengthened about six years. During the first thirtyfive years of the past century, the vital statistics of the city of London showed a mortality of about 29 per thousand. At the present time the mortality in that great city has been reduced to from I 7 to 19 per thousand.

No doubt a considerable proportion of this reduction in the rate of mortality in London and in other large cities in this country and in Europe is due to a diminished typhoid-fever rate. Indeed, sanitarians at the present day depend largely upon this factor of the general mortality rate of cities as an index of their sanitary condition. The following table showing the typhoid death-rate of cities, compiled by the Register of Vital Statistics of the New York Health Department, is given in a recent publication.

\section{AMERICAN CITIES}

\begin{tabular}{|c|c|c|c|}
\hline & Population. & Deaths. & $\begin{array}{c}\text { DEATH-RATE PER } \\
\text { IO,000 INHABI- } \\
\text { TANTS. }\end{array}$ \\
\hline Washington........... & 278,718 & 161 & $5 \cdot 78$ \\
\hline Chicago............. & I, 698,575 & 509 & 3.00 \\
\hline Boston .............. & 573.579 & 142 & 2.48 \\
\hline Philadelphia ......... & $\mathbf{I}, 321,408$ & 444 & $3 \cdot 3^{6}$ \\
\hline Providence ........... & 178,000 & 47 & 2.04 \\
\hline New York............ & $3,536,517$ & 727 & 2.06 \\
\hline St. Louis . . . . . . . . . & 598,000 & 198 & $3 \cdot 3^{I}$ \\
\hline San Francisco.......... & 360,000 & 70 & I.94 \\
\hline
\end{tabular}




\section{FOREIGN CITIES}

\begin{tabular}{|c|c|c|c|}
\hline & Population. & Deaths. & $\begin{array}{c}\text { DEATH-RATE PER } \\
\text { IO,OOO INHABI- } \\
\text { TANTS. }\end{array}$ \\
\hline Belfast ............. & 351,083 & 349 & 9.94 \\
\hline St. Petersburg......... & $\mathbf{I}, 248,643$ & 1,060 & 8.49 \\
\hline Cairo................ & 608,910 & 485 & 7.96 \\
\hline Glasgow............ & 764,467 & 198 & 2.59 \\
\hline Liverpool......... & 686,454 & I65 & $2.4 I$ \\
\hline Dublin .... & $37^{6}, 08 \mathrm{I}$ & 104 & 2.77 \\
\hline London ... & $4,544,983$ & $54^{8}$ & I. 2 I \\
\hline Paris...... & $2,660,559$ & 343 & I. 29 \\
\hline Berlin ... & $\mathrm{I}, 89 \mathrm{I}, 900$ & 88 & 0.47 \\
\hline Vienna... & $\mathrm{I}, 735,740$ & 76 & 0.44 \\
\hline Munich ....... & 503,000 & 24 & 0.48 \\
\hline
\end{tabular}

The high typhoid-fever death-rate in the city of Washington is probably due in part to the contamination of its water supply and in part to the fact that its sewer system is still incomplete. Congress has recently appropriated money for a sand-filtration plant, such as is now in use for London and various European cities, and it is hoped that within a few years, at least, the typhoid mortality may compare more favourably with that of London, Paris, Berlin, Vienna, and Munich, as shown in the above table.

The typhoid-fever death-rate per 10,000 of inhabitants in the city of Chicago was 9.16 in 1890 and I 7.38 in the following year. This, however, represents an unusual epidemic prevalence of the disease, which was continued into the following year (I2.06 per 
I0,000), after which a gradual reduction occurred until the census year 1900, when the very low rate of I.98 was reached. In New York the typhoid rate was 4.47 per 10,000 inhabitants in 1870 , and it has only slightly exceeded this figure in two years since that date ( 4.77 in $188 \mathrm{I}$ and 4.72 in 1883 ). Since 1893 it has been constantly below 2 per 10,000 inhabitants, a rate which probably to a large extent represents cases in which infection occurred outside of the city at summer resorts, etc.

In Philadelphia the typhoid death-rate has been constantly above that of New York, as might be expected from the superior advantages enjoyed by the last-named city in its water supply - from the Croton River. The highest typhoid death-rate recorded for the city of Philadelphia during the past thirty years was 9.22 per 10,000 in the year 1876 , the lowest 3.24 in the year I894. The average has been about 5.6, which is but little below the death-rate for the city of Washington in the year 1900 as given in the above table.

In Boston the typhoid mortality has fluctuated between 8.62 per 10,000 in 1872 and 2.55 in 1900 , the latter rate being the lowest recorded during a period of thirty years.

Without doubt a majority of typhoid-fever epidemics are due to infection of the water supply by 
typhoid excreta. A good example of a severe epidemic directly traceable to such contamination is afforded by the recent epidemic at Ithaca, N. Y., during which 9 I 2 cases with 58 deaths occurred during the months of January, February, and March, I903; 128 cases and 26 deaths occurred among the students attending Cornell University.

The fatal epidemic which occurred at Plymouth, $\mathrm{Pa}$, some years ago, has been traced to the pollution of the water supply by the excreta of a single case of typhoid fever, and this notwithstanding the fact that the water passed through three storage resęrvoirs, having altogether a capacity of 5,000,000 gallons. During this epidemic I IO4 cases were recorded with a mortality of I I 4 .

Without doubt typhoid fever is sometimes spread by means of infected dust containing living typhoid bacilli in desiccated excreta of typhoid patients. This is one of the ways in which the disease is spread among new levies of troops. It has been shown by experiment that the typhoid bacillus may retain its vitality when mixed with dry earth for a period of at least twenty-five days.

There is a good reason to believe that in several of our camps, during the Spanish-American War, this was an important factor in the etiology of typhoidfever epidemics. The average mortality from typhoid 
fever in our regular army since the Civil War has been: For the first decade (1868-1877), 9.5 per 10,000 of mean strength; for the second decade (1878-1887), 10.8 per 10,000; for the third decade (1888-1897), 5.5 per 10,000. This last rate compares favourably with that of many of our principal cities: for example, it is exceeded by the typhoid death-rate of the city of Washington, which is $7.8 \mathrm{r}$ per 10,000 (average of ten years, I888-1897); by that of the city of Chicago, which is 6.44 per 10,000; by that of Pittsburg, which is 8.8 per ro,000. As a result of unsanitary conditions existing in the camps in which our troops were hastily assembled at the outset of the Spanish-American War, the typhoid death-rate in our army of volunteers and regulars during the year ending April 30, I899, was more than twentytwo times as great as it had been in our regular army during the decade immediately preceding the war period. As compared with the Civil War, however, there was a decided improvement, the typhoid mortality for the first year of the Civil War having been 197. I per 10,000 of mean strength and for the Spanish-American War 123.7 per 10,000.

Experience shows that new levies of troops are especially subject to typhoid fever and other infectious "camp diseases," not only because of lack of discipline and consequent difficulty in the enforcement of 
sanitary regulations, but also because the individual soldiers are very susceptible to infection, owing to their age, the abrupt change in their mode of life, the exposure and fatigue incident to camp life, and last, but not least, their own imprudence as regards eating, drinking, exercise, etc. In the absence of sewers or other adequate means of removing excreta, the camp site is likely to become infected by the discharges of unrecognised cases of typhoid fever, and typhoid bacilli are carried by flies to the kitchen and mess-tents and deposited upon food, or as dust are directly deposited upon the mucous membranes of the respiratory passages of those living in the infected camp.

It must be remembered, also, that typhoid bacilli are frequently present in great numbers in the urine of typhoid-fever cases, and that this is a prolific source of infection of the ground under the conditions existing in camps. The presence of typhoid bacilli in immense numbers in the urine of convalescents from typhoid has been repeatedly demonstrated, and in view of this fact it is evident that measures of disinfection should be continued until all danger of propagating the disease in this way has passed.

The outbreaks of typhoid fever in cities, which have frequently been traced to infected milk, and in which the cases are often limited to those who use the milk from a particular dairy, are primarily due to 
infected water used to dilute the milk or to wash the vessels containing it.

The bacillus of typhoid fever is a rod-shaped microorganism, about one fifty-thousandth of an inch thick (from five- to eight-tenths of a micro-millimetre) and from two to six times as long as it is broad; it also

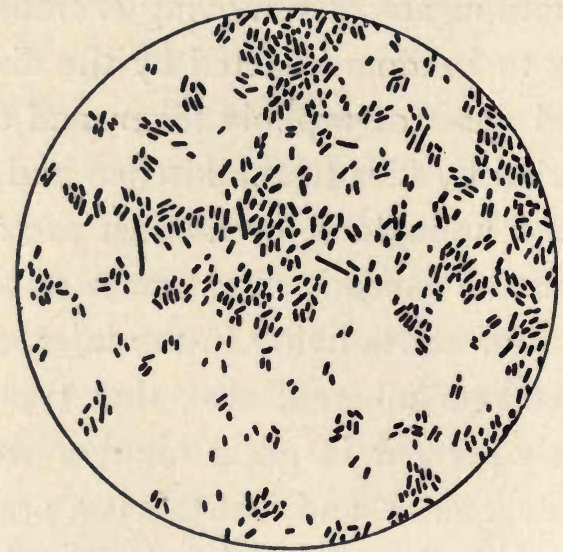

FIG. 3. Bacillus of typhoid fever; magnified I000 diameters.

grows out into long thread-like filaments. The minute size of this and other similar disease germs will perhaps be better appreciated when we say that one cubic centimetre of urine from a typhoid patient has been found to contain as many as $172,000,000$ typhoid bacilli-estimated by a counting method well known to bacteriologists.

This bacillus was first observed in 1880 by the German physician and bacteriologist, Eberth, and in- 
dependently by Koch. That it is the veritable germ of the infectious disease known as typhoid fever has been demonstrated by subsequent researches and is now generally admitted. This bacillus grows readily in milk, in beef tea, and in other culture media usually employed by bacteriologists. It is killed by a few minutes' exposure to a temperature of $140^{\circ}$ Fahr. $\left(60^{\circ} \mathrm{C}\right.$.), but unlike the cholera spirillum is not readily destroyed by desiccation, having been preserved in dry and pulverised soil for a period of twenty-five days. It may preserve its vitality in sterilised water for four weeks or more, and in typhoid excreta for at least three months. It has been recovered by cultivation from earth, upon which bouillon cultures had been poured, after an interval of five and one-half months; and from soil which had been manured with the undisinfected discharges of typhoid patients after these discharges had remained in a pit during five winter months.

These facts make it apparent why typhoid fever has become so widely disseminated throughout the world and why it is the principal endemic filth disease in the United States. A primitive and sparse population, which obtains its drinking water chiefly from live springs and running streams, is less liable to suffer from this disease than the permanent denizens of towns which are destitute of sewers and which 
depend upon shallow wells or polluted streams for their supply of drinking water. The liability to dangerous soil pollution in the vicinity of human habitations, or in temporary camps, is so great that sanitarians have long since learned to look with suspicion upon wells located in towns or in the vicinity of privy-vaults in the country. Numerous examples are upon record of local outbreaks of typhoid fever directly traceable to the drinking of contaminated well-water.

The typhoid bacillus is promptly destroyed by the various chemical disinfectants named in the previous chapter as germicidal for the cholera spirillum. From a practical point of view, for the disinfection of excreta, we recommend especially milk of lime, carbolic acid (five-per-cent. solution), chloride of lime (four-percent. solution), lysol, cresol or creolin (two-per-cent. solution). The quantity of the disinfecting solution used should be in excess of the quantity of excreta to be disinfected, and the time allowed for the action of the germicidal agent should be at least two hours.

Boiling water in liberal amount (four or five times the quantity of liquid excreta and urine) may also be used with perfect safety and will accomplish the object in view within a few minutes (ten minutes). For bed linen and soiled underclothing, immersion in boiling water or in a two-per-cent. solution of one of 
the coal-tar products above mentioned is to be recommended (carbolic acid, lysol, creolin, or tricresol).

The prevention of typhoid fever by protective inoculations has been tested on a large scale, by surgeons of the English army, in India and in South Africa. Sterilised cultures of the typhoid bacillus are used for this purpose. The inoculation gives rise to local tumefaction and to more or less general disturbance of brief duration. It is recommended that a second inoculation be practised at the end of a week. Wright, the originator of the method, reports that among I I,295 British soldiers inoculated in India, the percentage of those who subsequently contracted typhoid fever was 0.95 , while 2.5 per cent. of those not inoculated suffered an attack of the disease. The soldiers in South Africa, during the Boer War, who had been inoculated are reported to have suffered attacks of typhoid in the proportion of six per thousand, while those not inoculated suffered to the extent of nine per thousand. The difference is not sufficiently great to give confidence in inoculations by Wright's method as a reliable preventive measure. In this disease, as in cholera and bubonic plague, our main reliance should be upon the sanitary measures heretofore referred to, and especially upon disinfection of excreta and sterilisation of drinking water. 


\section{CHAPTER IV}

DYSENTERY, CHOLERA INFANTUM, ETC.

$\mathrm{D}^{\mathrm{y}}$ YSENTERY and the fatal form of "summer complaint," so common among the poorer classes in cities, are due to a bacillus which resembles in many respects the bacillus of typhoid fever, but which nevertheless presents certain specific differences which can be recognised by the expert bacteriologist. As this bacillus is found in the excreta of the sick and an attack of either disease depends upon the introduction of the germ to the intestinal tract, by way of the mouth, we must include dysentery and cholera infantum among the filth diseases. There is another form of tropical dysentery due to a parasite of a different class (amœbic dysentery), but this is also propagated in the same way and its prevention calls for the same sanitary measures. These are identical with the measures already recommended in previous chapters for the prevention of cholera and of typhoid fever. The victims of cholera in- 
fantum are no doubt usually infected through the medium of milk, which becomes contaminated either at the dairy or after it has been brought to the cityin some cases no doubt by flies which have been in contact with the discharges from the bowels of other patients similarly affected.

In Oriental countries where human excreta are used to fertilise the soil for growing lettuce, and other vegetables which are eaten without cooking, it is believed that dysentery is not infrequently contracted through the medium of such vegetables.

In tropical and semi-tropical countries dysentery claims more victims than typhoid fever, which prevails more extensively in regions lying within the temperate zone. Among English soldiers serving in India and China, more deaths occur from dysentery alone than from all diseases among troops at home stations.

United States troops in the Philippine Islands have suffered severely from tropical dysentery, which constitutes the principal cause of mortality.

The death-rate per thousand from "diarrhœal diseases" among our soldiers in the Philippines during the year I900 was 2.02, while in the United States it was O. I2. Epidemics of dysentery have, however, frequently occurred within the limits of the United States, and during our Civil War this and other forms of intes- 
tinal flux (chronic diarrhœa) caused a large share of the mortality among the troops, on both sides, in that momentous struggle. Deposits of infected excreta upon the surface of the ground or in shallow pits ; neglect of camp sanitation ; the mischievous activity of flies, which often constitute a veritable pest under such conditions; contamination of the water supply; and the deposit of dust from the infected camp site upon the moist mucous membrane of the air passages, are the chief factors in the propagation of all the infectious filth diseases - that is, in those diseases in which the disease-producing germ is present in the excreta. We might include here certain diseases due to the presence of parasitic worms in the intestine, the ova of which are discharged with the excreta. Recent researches show that a disease characterised by persistent anæmia and debility and often ascribed to "malaria," which is very common in many of the Southern States, is due to a minute intestinal worm (Uncinaria americana). Dr. Stiles, of the Public Health and Marine Hospital Service, to whom we owe this discovery, says : "I have now examined specimens of this new parasite from Virginia, Texas, Cuba, and Porto Rico. The clinical picture of the disease does not differ from that of the malady caused by the Old World parasite Uncinaria duodenalis."

The vast importance of the measures recommended 
for the prevention of the various filth diseases heretofore referred to will be appreciated, when the fact is taken into consideration that in our great Civil War nearly as many deaths resulted from these diseases as from wounds inflicted in battle.

During the Civil War the number of deaths from disease in the Union army was 186,216 ; the number killed in battle or who died of wounds was 93,969 . The number of deaths from the several forms of diarrhœea and dysentery was $44,55^{\circ}$, the number due to " camp fevers," including typhoid, " typho-malarial," etc., was $40,656,-\mathrm{a}$ total of 85,214 . This is also the history of wars in other countries and in all agesdisease claims more victims than battle. When we add to the deaths resulting from filth diseases in the armies of the world the enormous mortality which has occurred and continues to occur among the civil population, the figures are astounding. And yet these diseases are now generally recognised as "preventable diseases" ; and, as shown in previous chapters, great success has already attended the efforts made by sanitarians to restrict their prevalence. In the United States army, during the Spanish-American War, typhoid fever prevailed extensively in nearly all our camps among the new levies of troops, but within a few months its ravages were checked by the enforcement of proper sanitary measures. In the 
Philippine Islands, owing to the difficulties encountered in the execution of well-known preventive measures, there has been considerable mortality from filth diseases. But when we consider that our troops have been engaged in active operations in a tropical country, where all forms of intestinal flux are prevalent, and where they have been more or less exposed to infection by the germs of cholera and bubonic plague as well as by that of typhoid fever, it is safe to say that the sanitary record of our army in the Philippines is unsurpassed by that of any other body of troops which has been exposed in a tropical country under similar conditions. 


\section{CHAPTER V}

\section{RELAPSING FEVER}

RELAPSING fever is one of the pestilential diseases which has contributed considerably to the mortality of Europe, especially during times of famine. It was not clearly recognised as a distinct disease until the eighteenth century, and it is, therefore, impossible to say to what extent it may have prevailed prior to that time.

The first clearly recorded epidemic occurred in Ireland in 1739 , but the literature relating to the disease belongs mostly to the nineteenth century. The fact that the disease was not recognised previously to the date above mentioned cannot be taken as evidence that it did not exist. Typhoid and typhus fevers had not been differentiated as distinct diseases at this time; and, as all of these diseases are likely to prevail simultaneously when insanitary conditions exist, especially in densely populated countries and in times of scarcity of food, it is probable that all of these 
diseases had a share in contributing to the mortality statistics of Europe and Asia during the Middle Ages and in earlier times.

Relapsing fever prevailed in Ireland and in Scotland during the years 1799-1800, 1817-19, 1826$27,1842-48$, and in the last year (1848) it invaded several of the larger towns of England. In I86870 it again prevailed in England and Scotland, and cases are reported to have occurred in London as recently as the year 1873 . On the Continent the first accounts we have come from Russia-Odessa, in I833; Moscow, 1840-41. In the autumn of 1863 the disease reappeared in Odessa ; the following year it became epidemic over extensive areas in Russia, and extended to Livonia and Finland (1865), to Siberia (I866), and to Poland (I868). According to Hirsch, the disease continued to prevail in Russia over extensive areas during subsequent years, and was observed among the Russian troops as late as 1878-79. In Germany an extensive epidemic broke out in 1868 , as a result of importation from Russia. A second, more restricted, epidemic occurred in $187 \mathrm{I}-72$, and a third in $1878-79$. In the west and south-west of Europe-Switzerland, France, Italy, Spain-the disease is as yet unknown. In India relapsing fever has, no doubt, prevailed for many years, but the differential diagnosis between it and remit- 
tent fever, or the specific continued fevers which prevail there so largely, was not clearly made out by the earlier observers. During the last thirty years, however, numerous outbreaks of this disease in various parts of India have been recorded, and Carter has demonstrated that the disease, as it occurs in that country, is identical, as regards its clinical history, with relapsing fever, as described by recent European authorities; and, also, that it is characterised by the constant presence of the spirillum discovered by Obermeier in blood drawn during a febrile paroxysm. Relapsing fever has several times been imported to the United States, but its prevalence has been limited to restricted areas in our largest seaport cities. In 1844 fifteen cases were received into the Philadelphia Hospital from an emigrant ship sailing from Liverpool; in I 848 a few cases arrived in New York; and in $1850-51$ Dr. Austin Flint saw a number of cases, among recently arrived Irish emigrants received into the Buffalo City Hospital. But no epidemic resulted from these importations, and it was not until some years later (1869-70) that the disease became epidemic in certain sections of the cities of New York and Philadelphia. Parry, who made a careful investigation with reference to the origin of the first cases in Philadelphia, was unable to trace them to importation; but this can scarcely be questioned, in view of 
what is known of the history and etiology of the disease, and in consideration of the fact that Philadelphia is a seaport city which has constant communication with ports on the other side of the Atlantic, which at that time were known to be infected. The number of cases reported during this epidemic in Philadelphia was 1 I 76.

The coincidence of relapsing fever and typhus fever has been noted in many of the epidemics which have occurred in Europe, but the history of this coincidence does not justify the supposition that there is any etiological relation between these diseases other than that furnished by common predisposing causes, viz., the depressing effects of overcrowding, insufficient food, and filthy surroundings. This view is supported by the fact that either disease may occur alone, and the circumstance that sometimes one and sometimes the other has the precedence in time in those epidemics in which coincidence has been observed.

The germ of relapsing fever was discovered in 1873 by Obermeier, a German physician. This was the first discovery of the specific germ of an infectious disease peculiar to the human race, and opened the way for the subsequent demonstration of the germs of leprosy (I879), typhoid fever (I880), pneumonia (1880), tuberculosis (1882), cholera (1884), 
diphtheria (1884), tetanus (1884), influenza (1892), bubonic plague (I894), dysentery (1900).

This germ is a slender spiral filament, which is found in the blood of relapsing-fever patients during the febrile paroxysm. The presence of the spirillum in the excreta of relapsing-fever patients has not been demonstrated, but it can scarcely be doubted

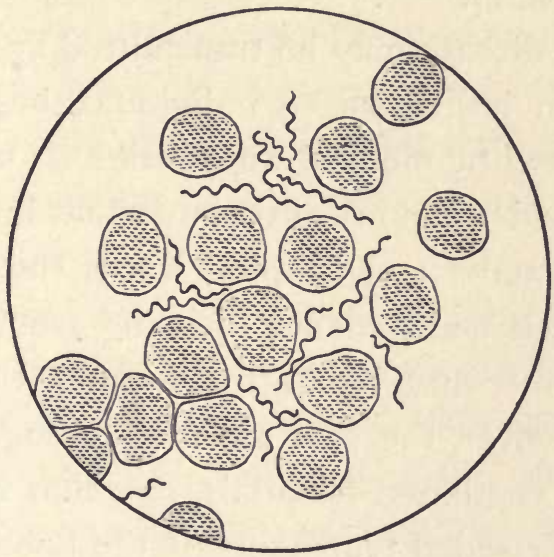

FIG. 4. Spirillum of relapsing fever, as seen in the blood; magnified rooo diameters.

that this disease belongs in the same category with typhoid fever, bubonic plague, and Asiatic cholera$i$. e., that it is one of the filth diseases and is spread principally by means of excreta containing the specific infectious agent or germ. It may be that the spirillum of relapsing fever produces reproductive spores, which would be difficult of demonstration in 
the excreta. Even the spirillum itself is not easy to see. A good microscope and a considerable degree of skill in its use are required for the demonstration of the slender spiral filaments in blood drawn from the circulation of a relapsing-fever patient. Although comparatively long and endowed with active movements, they are only about one fifty-thousandth of an inch in diameter.

That the disease may be transmitted by direct contagion, from individual to individual, has generally been admitted by medical authorities. On the other hand, the evidence on record shows that in well ventilated apartments and hospital wards the attendants upon the sick and patients suffering from other diseases are not very liable to contract the disease. Where, however, the sick are massed together in insufficiently ventilated hospitals, or when cases occur in the overcrowded tenements of the poor, the transmission of the disease to attendants and others exposed to contagion is far more frequent.

$\mathrm{Up}$ to the present time, attempts to reproduce the spirillum of relapsing fever in a series of culturcs have not been successful.

There is no evidence that climate or season has any marked influence upon the prevalence of relapsing fever; the disease has prevailed in Siberia as well as in India, and its preference for certain localities is 
quite independent of climatic conditions, relating rather to circumstances connected with the mode of life and hygienic surroundings of the population. No age is exempt, and sex has no apparent influence ; but children are more subject to be attacked than adults, and susceptibility seems to diminish to some extent with advancing age. According to Murchison, only 195 out of 2 I I cases received into the London Fever Hospital, in twenty-three years, were over fifty years of age. To appreciate the value of these figures it would evidently be necessary to know how large a proportion of the exposed population were over fifty years of age.

Insufficient food is generally recognised by medical writers as a potent predisposing cause, and epidemics have so frequently been observed to coincide with periods of unusual scarcity that the name "famine fever" has been applied to the disease. Some authors have even gone so far as to ascribe to starvation and its accompaniments - overcrowding and filthy surroundings - an essential rôle in the development of the disease. But, as in the case of other specific contagious diseases, there seems to be very little foundation for the idea that relapsing fever may be developed de novo in times of famine, and its epidemic prevalence at such times is to be ascribed rather to increased vulnerability, on the part of the starving 
population, to the action of the specific exciting cause of the disease. We know that under favourable hygienic conditions the disease has but little disposition to spread, and that in the severest epidemics it finds its victims almost exclusively among the destitute. On the other hand, in the numerous instances in which shipwrecked mariners, Arctic explorers, etc., have been subjected to absolute starvation, we have no account of the development of any such disease as relapsing fever. Overcrowding is considered by Parry to be a more potent predisposing cause than starvation, and his careful study of the circumstances of those who were taken sick during the prevalence of the disease in Philadelphia (1870) seems to justify this conclusion - which is, moreover, supported by the observations of Muirhead, Bennett, Lebert, and others.

One attack of relapsing fever does not protect the individual from subsequent attacks, and second or even third attacks during the same epidemic have been noted.

What has been said with reference to the factors which favour the epidemic prevalence of the disease will indicate the proper preventive measures. Modern sanitary science has, apparently, banished this disease from its former haunts in European countries. It has not been introduced into the United States 
since the epidemic heretofore referred to, which occurred in the city of Philadelphia in the years 186970. Should cases of the disease again be brought to our shores, prompt isolation of the sick and the disinfection of excreta would probably suffice to prevent its extension 


\section{CHAPTER VI}

\section{TYPHUS FEVER}

$T$ YPHUS fever, also known as "spotted fever," has doubtless prevailed in Europe for many centuries, although no definite account of a disease which can be identified with typhus is known of an earlier date than the eleventh century. It was not, however, until the sixteenth century that the disease was described in a tolerably satisfactory manner by the Italian physicians ( 1505 to 1530 ). Epidemics of typhus have frequently been associated with the devastations of war and the scarcity of food resulting from such devastations; or from failure of crops, which is one of the principal predisposing causes of the disease.

During the eighteenth century the disease prevailed extensively in the various countries of Europe and especially in England, in which country three severe epidemics occurred. In two of these (1718-2I and $1728-31)$ the disease was widely prevalent in England 
and Scotland. During the ten years following I 734, and again in 1757 to 1775 , the disease prevailed in the track of contending armies over a large part of eastern and central Europe. The last mentioned period includes the time of the Seven Years' War, and of the war between England and Spain. At the end of the eighteenth and the beginning of the nineteenth centuries typhus was again very prevalent in Europe. Hirsch, in his Handbook of Geographical and Historical Pathology, says with reference to this period :

"The fourth and by far the severest period of typhus in the eighteenth century occupies the last ten years of it; it begins with the revolutionary wars on French soil, and ends, in the second decade of the present century, with the final retreat of the French army across the Rhine, the overthrow of the empire of Napoleon, and the restoration of peace."

Widespread epidemics of typhus in Europe ceased with the ravages attending the wars of Napoleon, with the exception of the years $1846-47$ when the disease was somewhat widely diffused. In Ireland and in certain other parts of Europe the disease is apparently endemic, and any unusual period of distress is apt to be followed by an epidemic. In America the disease has prevailed in Mexico from an early date and is said to be endemic in the city of Mexico and other localities in the interior. It has also been 
of frequent occurrence in Chili and in Peru. The disease has frequently been introduced into the United States by immigrants from Europe, and local epidemics have resulted from such importation, especially in New York (1818, 1827, 1837, 1847) and in Philadelphia (1827, 1835, 1847). Fortunately the disease is unknown in other portions of the United States, probably because our history does not include any famine periods, such as have apparently constituted an essential factor in the epidemic prevalence of the disease in European countries. Africa and Asia have been to a great extent exempt from this scourge, although it has prevailed in northern Africa, in Persia, and in Syria. Typhus is essentially a disease of temperate and cold countries. In the populous countries and islands of the tropics it is practically unknown. Epidemics occur more frequently during the winter and spring months than in summer. This is probably due to the fact that it is personally contagious. Like other diseases which are communicated from person to person, it is more likely to extend when cold weather leads to an indoor life and more intimate and continued association of individuals. Again, the fact that scarcity of food is more likely to occur in winter and in the early spring is probably a factor of some importance in determining the seasonal prevalence of the disease. 
The influence of insanitary conditions in favouring the epidemic extension of typhus is generally admitted. This is so well stated by Hirsch that I cannot refrain from again quoting from this trustworthy author:

"It is always and everywhere the wretched conditions of living, which spring from poverty and are fostered by ignorance, laziness, helplessness, in which typhus takes root and finds nourishment ; and it is above all in want of cleanliness, and in the overcrowding of dwellings, that are ventilated badly or not at all, and are tainted with corrupt effluvia of every kind. The prototype of these conditions is found in Ireland, which is the greatest sufferer from this disease."

The same conditions as to overcrowding, want of ventilation, and insanitary surroundings have been the determining factors in the development of epidemics in prisons, on shipboard ("ship-fever"), and among colonies of workmen brought together to prosecute great engineering enterprises (canals, railways, etc.), and not provided with proper quarters. When poorly housed and left to their own resources, labourers or soldiers will inevitably develop insanitary conditions in and around the barracks, huts, or tents occupied by them, and some pestilential malady will just as inevitably obtain a foothold among them, with more or less disastrous consequences.

The germ of typhus fever has not been discovered, but it is an eruptive fever in which, as in smallpox, the infectious agent is probably given off from the 
entire surface of the body. At all events it is very contagious, and the infection clings to clothing and other articles which have been in contact with the sick. This being the case, our chief reliance for the prevention of the disease must be on the isolation of the sick, and careful disinfection of all articles that have been exposed to infection and of the patient's body and excreta. This is to be accomplished by the same measures recommended in the case of smallpox.

Owing to the fact that typhus fever is communicated by personal contact, and in the absence of any known method of establishing immunity by inoculation, physicians and nurses are especially liable to contract the disease. During the epidemics in Ireland, between the years I 8 I 3 and 1848 , it is reported that 568 physicians contracted typhus fever and of these 132 died. During the Crimean War, the mortality from this disease, among physicians and nurses, was very great.

One attack of typhus usually confers immunity upon the individual, but second attacks may occur. The mortality from the disease is slight among children under ten years of age, but increases with age, being highest between the ages of thirty and fifty $(41.97 \%)$. 


\section{CHAPTER VII}

TUBERCULOSIS

$\mathrm{I}^{\mathrm{T}}$ is a well-established fact, which is generally recognised, that pulmonary consumption is due to a minute bacillus, discovered over twenty years ago by the famous German bacteriologist, Robert Koch, and that this bacillus is present in large numbers in the purulent matter coughed up from the lungs of those suffering from the disease. We also know that the propagation of the disease depends largely upon the introduction of the bacillus, suspended in the inspired air in the form of dust, to the lungs of susceptible individuals, by way of the respiratory passages. That dust in hospitals and dwellings occupied by consumptive patients may contain living tubercle bacilli has been repeatedly demonstrated, and it is evident that the only way to guard against this danger is to destroy by fire or disinfecting agents all expectorated material coming from the lungs of consumptive patients. If this is done, such persons do not endanger those with whom they are associated; if it is not done, 
they become active agents in the transmission of this infectious disease - " the great white plague"-which is responsible for twelve per cent. of the deaths which occur within the limits of the United States.

The last census report shows that in the census year, 1900, 109,750 deaths occurred from pulmonary consumption. This is a smaller number in proportion

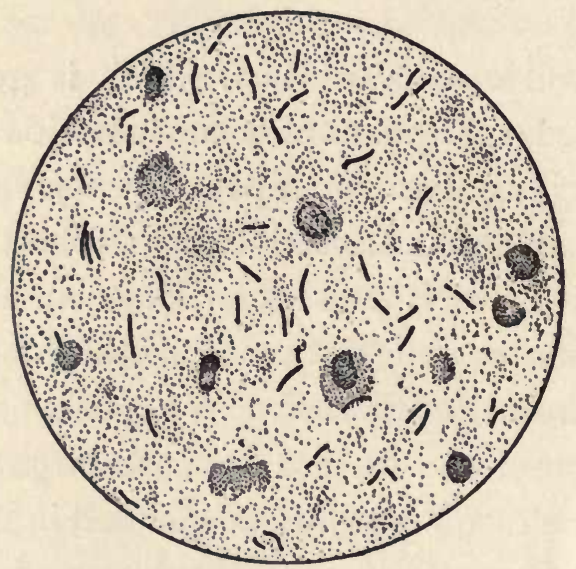

FIG. 5. Bacillus of tuberculosis, as seen in the sputum of a patient having pulmonary consumption; magnified rooo diameters.

to the population than occurred in 1890 . A decided gain is shown during recent years by the mortality statistics of some of our large cities, in which special efforts have been made to instruct the public as to the method in which the disease is propagated, to prevent expectoration in public places, and to disinfect the sputa of recognised cases of pulmonary tuberculosis. New York City, under the able leadership of Dr. 
Hermann M. Biggs, Medical Officer of the Department of Health, has taken the lead in carrying out these measures for the prevention of the disease, and with most notable results. The death-rate from tuberculous diseases has been gradually and progressively reduced from 4.42 per cent. in 1886 to 2.89 per cent. in I902. Dr. Biggs says in a recent paper: "During the first six months of 1902, 4673 deaths from tuberculosis occurred, equalling a reduction of Io per cent. on the death-rate of Igor, which was the lowest ever recorded."

As to the methods adopted in New York, Dr. Biggs says :

"In I 888 a commencement was made by issuing circulars setting forth the essential facts regarding the nature of the disease, the usual methods of its transmission, and the precautions necessary to prevent its spread. Small circulars of information were also freely distributed in the tenement-house districts and the public press was utilised for the education of the public as to the communicable nature of tuberculosis. This campaign of education was continued until 1893 when the City Board of health required all public institutions to promptly report all cases of pulmonary consumption coming under observation and requested physicians in private practice to do the same. In 1897 a section was added to the Sanitary Code declaring pulmonary tuberculosis to be an 'infectious and communicable disease, dangerous to the public health,' and requiring physicians to report to the Health Department all cases coming under their observation."

Dr. Biggs concludes his paper, from which I have quoted, as follows : 
"It seems evident that sure progress is being made, and with the cordial support of the medical profession, as a whole, which is now freely accorded by the best elements of the profession, followed as this must be by the universal popular appreciation of the true nature of tuberculosis in place of the unreasoning fear or careless indifference which has largely prevailed, we may confidently anticipate, as the result of continued effort, the almost entire eradication of this, the most fatal disease with which sanitary authorities have to deal."

The last census return (I900) shows that in the registration area the death-rate from consumption was 187.3 per 100,000 of the population, while the preceding census gave a rate of 245.4 per 100,000 .

Similar progress is reported in those countries of Europe which have given the most attention to sanitary matters. In Prussia a notable diminution in the mortality from tuberculosis commenced about the year 1887 , five years after the discovery of the tubercle bacillus. This has been continuous up to the present time. Prior to the date mentioned, the mortality rate from tuberculosis in Prussia had for some time remained stationary at about 310 per 100,000 inhabitants; since the year 1887 , it has fallen to 210 (in the year 1900). Similar progress in the future would lead to the practical extinction of the disease in twenty or thirty years. In England, during the same period, a reduction from 240 to 190 per 100,000 has occurred. The last census return shows that the District of Columbia has "the highest death-rate 
(305.3) from consumption in the registration States." This is ascribed to the large proportion of coloured population.

Recent researches have led Professor Koch to the conviction that tuberculosis is more often communicated by means of the minute drops of sputum forcibly ejected by consumptive patients during paroxysms of coughing than by the masses of sputum which have been coughed up and expectorated. However this may be, there can be no doubt that the main source of infection is to be found in the sputum of persons suffering from pulmonary tuberculosis. In tuberculosis of the bowels, tubercular meningitis, and tubercular joint affections, the patient must have been infected by such material, or by tubercle bacilli in articles of food, such as milk or meat from tuberculous animals. But such cases do not assist in propagating the disease. An infant, crawling upon the floor of an infected house, is especially liable to infection through the medium of the dust, which naturally settles upon the floor, and to develop tubercular meningitis, which is the form of tubercular infection to which young children are most subject. Older children are more likely to contract tubercular joint disease- "hip disease," or "white swelling" of the knee joint," or tubercular disease of the spinal column ("Pott's disease"). The child with tubercular 
meningitis or tubercular peritonitis is usually doomed to death, but many children recover from the other forms of tuberculosis just mentioned and may grow up to be useful citizens, although the results of the disease from which they suffered are manifest in the stiff knee, immovable hip joint, or deformed spinal column.

Susceptibility to pulmonary tuberculosis is much greater in young adults than in children. This susceptibility depends upon several factors which constitute the predisposing causes. First we may mention an inherited weakness of constitution, or conformation of body-narrow chest, insufficient lung capacity, feeble circulation, imperfect digestion. Second come the conditions relating to an unfavourable environment-living in ill-ventilated or overheated rooms, absence of sunlight and dampness in apartments occupied, insanitary surroundings, overcrowding of living- and sleeping-rooms. Next we must place lowered vitality as a result of other acute or chronic diseases or vicious habits, and the catarrhal inflammation of the bronchial mucous membrane which is a prominent feature of certain other infectious diseases -especially measles, whooping-cough, and influenza -or which may result from "taking cold." Finally, occupation has much to do with establishing a predisposition to the disease. 
The following table, compiled from the last census return (1900), gives the death-rate per 100,000 from this disease for persons of various occupations :

Bankers, brokers, and officials of companies.... 92 Laundresses....................... 94

Farmers and farm labourers............ I II.7

Clergymen....................... 1 23.5

School-teachers, female.............. 126

School-teachers, male................. I44

Railroad employees................. 1 29.8

Dressmakers and seamstresses............ 130

Policemen...................... 1 36.7

Lawyers........................ 139.9

Mill operators..................... 144

Physicians..................... 158.8

Gardeners and florists.............. I 86.6

Journalists................... 188.4

Carpenters ...................... 23

Masons......................... 293

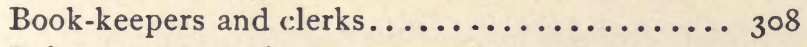

Painters and glaziers.................... 319

Servants, female........................ 319.7

Sailors and fishermen ............... 333

Barbers............................ 334.9

Cabinet-makers ........................ 359

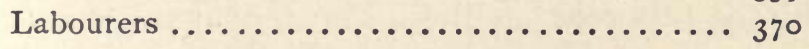

Servants, male................... 430

Printers ......................... 435.9

Cigar-makers...................... 476

Marble- and stone-cutters............. 540

Occupations in which the air is filled with dust particles, which irritate the bronchial mucous membrane, 
and are often deposited in the lungs in considerable quantities, constitute a predisposition to infection, as is shown by the high death-rate among marble- and stone-cutters.

The number of deaths from consumption both in this country and in England is greater among males than among females. In the United States, the total number of deaths from this disease, reported as occurring in the registration area during the last census year, was 53,962 , of which 29,192 were males and 24,770 females.

The Imperial Health Board of Berlin has issued the following statistics regarding the mortality from phthisis in Europe: Russia has more than 4000 deaths per 1,000,000; Austria-Hungary and France, more than 3000 per I,000,000; Sweden, Germany, Switzerland, and Ireland, more than 2000 per I,000,oo ; Netherlands, Italy, Belgium, Norway, Scotland, and England, more than 1000 deaths per I,000,000.

Certain races seem to be especially liable to infection by the tubercle bacillus. The census return (1900) shows that among those whose mothers were born in Ireland the rate was 339.6 per 100,000 , in France 184.7, in Scotland 172.5 , in the United States I 12.8 , in Russia and Poland 7 r.8. The comparative immunity of Russian and Polish Jews from pulmonary consumption had previously been established by the vital 
statistics of the city of New York for the six years ending in I890. It was found to be 76.72 per I00,$\infty 00$, while for Irish, during the same period, it was 645.73 , for coloured 531.35 , for Germans 328.8, and for native-born Americans 205.14. With reference to age, the statistics show a comparatively low rate below the age of fifteen, a rate of 252.4 between fifteen and forty-four, a smaller rate between forty-five and sixty-four years of age (232.5), and a maximum rate of 260 . I beyond the age of sixty-five.

While pulmonary consumption is an extremely fatal disease when those who contract it are obliged to live under the unfavourable conditions which contributed to its development, it is now well known that cases in the early stages of the disease are very amenable to suitable treatment, and that a considerable proportion of the cases recover under the influence of pure air and nourishing food. The fact that there is no specific medication for the disease is set forth in the following authoritative circular.

" THE COMMITTEE ON THE PREVENTION OF TUBERCULOSIS OF THE CHARITY ORGANISATION SOCIETY OF THE CITY OF NEW YORK

"Whereas, It has come to the knowledge of the Committee on Tuberculosis of the Charity Organisation Society that many so-called specific medicines and special methods of cure for tuberculosis have been and are being exploited and widely advertised, and 
"Whereas, The advertisements of some of these cures have made such reference to the Tuberculosis Committee of the Charity Organisation Society, or to some of its members, as to create the inference that this Committee, or its members, recommend or advocate the use of many such so-called specifics or special methods of cure for pulmonary tuberculosis, or consumption, and

"Whereas, There is no specific medicine for this disease known, and the so-called cures and specifics and special methods of treatment widely advertised in the daily papers are in the opinion of the Committee without special value, and do not at all justify the extravagant claims made for them, and serve chiefly to enrich the promoters at the expense of the poor and frequently ignorant or credulous consumptives, therefore,

"RESOLVED, That a public announcement be made that it is the unanimous opinion of the members of this Committee that there exists no specific medicine for the treatment of pulmonary tuberculosis, and that no cure can be expected from any kind of medicine or method except the regularly accepted treatment which relies mainly upon pure air and nourishing food.

Charles F. Cox, Chairman

OTTO T. BANNARD

Hermann M. Biggs, M.D.

HERBERT S. BROWN

JOSEPH D. BRYANT, M.D.

Miss Ella Mabel Clark

ROBERT W. DE FOREST

EdWARd T. Devine

HOMER FOLKS

FrankLiN H. GidDINGS

Henry Herbert, M.D.

J. H. Huddleston, M.D.

ROBERT HUNTER

A. JACOBI, M.D.

W ALTER B. JAMES, M.D.

E. G. JANEWAY, M.D.
Miss A. B. Jennings

S. A. KNore, M.D.

Alexander lambert, M.D.

ERNest J. Lederle

Mrs. Frederic S. LeE

Egbert Le Fevre, M.D.

Henry P. LoOmis, M.D.

Mrs. Jamfs E. Newcomb

Eugene A. Philbin

T. Mitchell Prudden, M.D.

ANDREW H. SMith, M.D.

W. G. Thompson, M.D.

E. L. TRUdEAU, M.D.

Frederick L. WACHENheim, M.D.

R. C. W. WADSWORTH

Miss Lillian D. Wald

Charles H. Johnson, Secretary. I05 East 22d Street." 
No doubt the favourable results as regards a diminution in the mortality from consumption, which have been obtained in England, Germany, and certain portions of the United States, are due in part to improved housing conditions among the poor, and in part to the removal of consumptive patients to sanatoria, rather than to the disinfection of sputa, which is still very imperfectly carried out.

The treatment of cases of pulmonary tuberculosis in special hospitals or sanatoria has a twofold advantage. It is in the interest both of the patients and of the general public. It has been demonstrated that under proper treatment in well-located sanatoria a considerable proportion of the cases may recover, and that the methods adopted in such sanatoria for the destruction of the sputa of tuberculous patients prevents the infection of the buildings and grounds.

On the other hand, private dwellings, especially among the poorer classes of the community, are almost sure to become infected by the prolonged residence in them of a case of pulmonary consumption, and sad experience shows that a first case is often followed by a series of cases, which, occurring in one family, were formerly ascribed to heredity. The persistent infection of localities in which cases have occurred is illustrated in the vital statistics of every city. Thus in New York City " on a single street 
block as many as 102 cases have been reported within a period of $4 \frac{3}{4}$ years and as many as 24 cases in a single house." If we were speaking of bubonic plague and the same number of cases had occurred within the same period, the whole country would be alarmed and the most rigorous efforts would be made to eradicate the disease, but "the great white plague" continues to claim its annual quota of from I 10,000 to I 50,000 victims within the limits of the United States, and the known measures of prevention are either entirely neglected or carried out in a very inadequate manner in a large portion of our great and prosperous country.

For the prevention of tuberculosis, as already indicated, the most essential sanitary measure is the destruction of the tubercle bacillus in the sputa of those suffering from pulmonary consumption. This may readily be effected when the victims of the disease and those associated with them become convinced of its importance. For this reason well-informed physicians and sanitary officials consider the education of the public a matter of prime necessity. It is hardly necessary to say that the sputa of tuberculous patients should be deposited in some suitable vessel and never upon the floor or ground. Regulations prohibiting expectoration upon the sidewalks or upon the floor in public vehicles, etc., have been 
adopted in several cities in the United States and should be enforced everywhere, not only in the interest of the public health but as a matter of decency. There was a time when all kinds of filth could be thrown into the streets of cities without danger of police interference. But the public has long since been educated beyond the point of submitting to such nuisances, and the time is not far distant when no self-respecting citizen will deposit masses or pools of expectorated matter in public places any more than he would upon the floor of his own drawingroom. Those who have no self-respect or regard for the sensibilities of their neighbours should be restrained by suitable laws.

When infected saliva has been deposited in a suitable receptacle of porcelain or of metal it is a simple matter to destroy it. This may be readily accomplished by placing the receptacles with their contents in boiling water for at least five minutes. A fiveper-cent. solution of carbolic acid or one of the other coal-tar disinfectants (lysol, creolin, tricresol) may be used for the same purpose, and it is a good plan to have the sputum directly deposited in a porcelain cuspidor containing such a solution. This should be emptied and cleaned in boiling water at least twice a day. Various forms of cuspidors have been devised for tuberculous patients and are to be obtained from 
dealers in medical and surgical appliances. Those which are made of enamelled iron, tin, or aluminum have the advantage of not being breakable. They should be provided with a cover to exclude flies and hide the contents from view. Pasteboard cups, which can be placed inside of a metal cup with cover, are especially to be commended as they can be removed and burned, together with their contents, at stated intervals.

Pocket flasks of aluminum are made for the use of tuberculous individuals who are not confined to the house. Dr. Knopf, of New York, in his valuable article in the Twentieth Century Practice of Medicine, makes the following remarks with reference to pocket flasks :

"The pocket flask, when properly and faithfully used by the pulmonary invalid, will prove one of the most important factors in the prevention of tuberculosis. It should be carried by the tuberculous individual all the time and used whenever he cannot conveniently get at the stationary cuspidors. The first pocket spittoon was Dr. Dettweiler's 'Hüstenflaschen.' It is a flask of blue glass about four inches long and six inches in its largest circumference, provided with a hermetically closing top and bottom of white metal, and so constructed that it can easily be cleaned. The lid flies open at a slight pressure on the spring, and after use is closed by pushing the top down again. Since the appearance of this flask many similar utensils have been devised, partly with a view of simplifying the mechanism and also of producing an article cheap enough to be within the reach of every one. 
"In the endeavour to attain these ends I have designed two models. The better of these, perhaps, is the aluminum flask constructed like Dettweiler's, but without the extra opening at the bottom.

"It is lighter, less bulky, and unbreakable. It is easy to disinfect as it can be boiled without injury. It possesses every advantage except that of cheapness. The other is a strong glass flask modelled like the aluminum flask just described. The funnel is of vulcanised rubber and the cover of white metal. The cleaning and disinfection are simple, and its cost is only about one-third of that of the aluminum flask. . . . All of these flasks are so constructed that the contents cannot be spilled when tipped over, even when the cover is open. . . . The patient should have two pocket flasks, so as never to be without while one is being cleaned."

The reporting of cases of pulmonary tuberculosis, by their attending physicians, to the health authorities, should be required, not with a view to isolation of the patient, as is demanded in the case of acute infectious diseases, but in order that the patient and his friends may receive reliable information with reference to the danger of the transmission of the disease and the method of preventing it. In New York City, which has taken the lead in this country in preventive measures, the method of procedure is stated by Dr. Hermann M. Biggs, Medical Officer of the Health Department, as follows :

"With certain exceptions, such as patients under the care of private physicians, all reported cases, living or dead, are assigned to the medical inspectors of tuberculosis for the district 
from which the cases are reported. The inspector visits the ad. dress given, and, if the patient is living, leaves a circular of information and gives verbal instruction to the friends about the danger of infection and the care of the sputum. If the address is that of a dead patient, or if the patient, though living, has moved, the inspector examines the premises and makes such recommendations as seem to him necessary to render the habitation free from danger of infection. These recommendations, made out on a prescribed form, usually advise the following routine treatment of apartments: Kalsomined or whitewashed walls or ceilings are washed with a solution of washing soda (onehalf pound to three gallons of hot water), and then kalsomined or whitewashed again; papered walls or ceilings are similarly washed and repapered; the woodwork is scrubbed with the soda solution and repainted. The inspector's recommendations are forwarded to the board, and on them as a basis a ten-day order is issued on the landlord requiring him to carry out the specified renovation. The execution of the order is then (as with all others of the board) placed under the supervision of the sanitary police. The premises are reinspected, and if, at the expiration of ten days, the owner has not complied with the order, it is referred to the attorney of the board for enforcement. This procedure was determined on because of the difficulty of disinfection of apartments in which cases of tuberculosis have been, and the greater efficiency attained by the system of renovation. The method is easy of enforcement, as is shown by the fact that compliance with the order has not been refused in a single case. The chief point of interest in this plan is that renovation rather than disinfection is called for. Disinfection involves the use of materials and methods which are not universally familiar. The method of renovation required is understood by all, and is more efficient than any method of disinfection which could be employed."

Prior to this renovation the room with its contents may be disinfected with formaldehyd gas, or 
articles of clothing and bedding which cannot be sent to the laundry may be disinfected by the Health Department, at its disinfecting station, either by steam or formaldehyd.

The danger of contracting tuberculosis through the medium of milk from tuberculous cows is to be guarded against by the killing of animals recognised as tuberculous and by the sterilisation of milk when it comes from a source not known to be free from suspicion.

That the tubercle bacillus is frequently, if not usually, present in the milk of tuberculous cows, has been demonstrated by numerous independent investigators.

We regard the danger of contracting the disease by eating the flesh of tuberculous animals as extremely remote. This should be condemned if the animal is found to have the lesions of pulmonary tuberculosis; but if these are not discovered, the facts that the bacilli are not located in the muscles, and that the meat is cooked before it is eaten, probably reduce the danger to an imaginary one.

It has been shown by experiment that tubercle bacilli may retain their vitality and infecting power in desiccated sputum for several months. Infected houses are therefore dangerous for a considerable time after they have been vacated by a consumptive 
person. The bacillus may also retain its vitality for one hundred days or more in putrefying material. This bacillus has also greater resistance to heat and various germicidal agents than most other pathogenic bacteria. But it is quickly killed by exposure to the temperature of boiling water; by a five-per-cent. solution of carbolic acid; by a two-per-cent. solution of chloride of lime; by milk of lime, solution of caustic potash (lye), or the mineral acids - a one-per-cent. solution of sulphuric or hydrochloric acid. Wood vinegar, or pyroligneous acid may also be used if at least six hours' time is allowed for disinfection.

What has been said with reference to predisposing causes will indicate the measures which should be taken to increase the resistance of individuals to infection-especially of those who have an inherited or acquired feebleness of constitution. An out-door life, systematic exercise, and a nutritious diet will be the main factors in establishing a relative immunity to the disease. Children with narrow chests and insufficient lung capacity should be required to take breathing exercises as prescribed by Dr. Knopf, of New York.

"The patient is taught to stand properly-that is to say, straight, chest out, and head erect-and to breathe always through the nose. He takes a deep inspiration slowly, beginning with the abdominal muscles, and then expanding the chest to its fullest 
capacity. During this inspiration he raises his arms from his sides to a horizontal position. He holds the breath for a moment and then lowers the arms during the act of expiration, which should be somewhat more rapid.

"The second exercise is like the first, except that the upward movement of the arms is continued until the hands meet over the head.

"In the third exercise the patient stretches his arms out as in the position of swimming, the backs of the hands touching each other. During the inspiration the arns are moved outwards and finally meet behind the back. They are brought forward again during the expiration. This exercise can be greatly facilitated and made more effective by the patient rising on his toes during the act of inspiration and descending during the act of expiration. Each respiratory act should be followed immediately by a secondary forced expiratory effort."

Exercises of this kind should not be taken soon after eating or when greatly fatigued. Abundant ventilation of sleeping- and living-rooms and sun-baths will do much to invigorate the system. When practicable, delicate children and individuals already infected by the tubercle bacillus should have the advantage of a favourable climate, such as that of portions of Colorado, New Mexico, or Arizona, where one may be out-of-doors nearly every day in the year, and where the continued sunshine and pure invigorating air often work wonders in the way of re-establishing the health of such persons.

To further enforce the statements made in this chapter, I take the liberty of quoting from a recently 
published paper by Dr. Hermann M. Biggs, Medical Officer of the Department of Health of New York City :

"Last year there were reported to the Department of Health more than 13,000 new cases. It may be safely estimated that this represents less than one-half of the cases actually existing in New York City, for many cases live for several years after they are brought to the attention of the Department, and are only included when originally reported, and many are not reported at all. For example, nearly 5000 other cases were reported in 1901 , which had been previously reported. I think we may safely estimate that 30,000 cases of tuberculosis in a stage of the disease in which it could be easily recognised by a competent physician are present in New York City.

"I have had a census of the cases actually under treatment in the hospitals in New York City made annually for a series of years, and the total number never much exceeded 1000, or less than 4 per cent. of the cases actually present in the city. The vast proportion of the remainder are in tenement houses. I have estimated that the total expenditure in the city of New York in its public institutions for the care and treatment of tuberculous patients is not over $\$ 500,000$ a year, or not more than 2 per cent. of the actual loss to the city annually. If this annual expenditure were doubled or trebled it would mean a saving of several thousand lives annually, to say nothing of the enormous saving in suffering.

"It is now fifteen years since the New York City Health Department first began, in a very small way, its efforts for the prevention of tuberculosis, and these have been rewarded by a reduction in the mortality out of all proportion to the expenditure in money and time which has been made. Still, more has been done in New York than in almost any city in the world. The measures, however, now in force are quite inadequate, as compared with the importance and magnitude of the problem. The sanitary authorities, however enthusiastic and efficient, and 
the medical profession, however influential and numerous, cannot grapple with this problem unless they have the hearty support of the people and the administration of the city. They must have generous appropriations for carrying on the work - for the provision of medical inspectors and disinfectors, for educational measures, for the establishment of dispensaries and sanatoria for the care of incipient cases, and of homes to which advanced cases may be removed, and where they may be made comfortable until the inevitable fatal termination comes. We must remember, in this connection, that every incipient case and every advanced case of tuberculosis which is removed from its home and surroundings and placed in a properly equipped and conducted institution is, in this way, not only given a fair chance for recovery of health, but is educated as to the means to be taken to prevent further extension of infection, and, at the same time, one focus of infection is removed from the city. On the average, every case of tuberculosis infects at feast one other case, and if removed to a hospital early enough, the infection of this second case would be, in each instance, prevented, and thus the total number of cases would be reduced.

"It is in an educational way that lay organisations for the prevention of tuberculosis may be of the greatest service. They serve to arouse interest in and to disseminate knowledge of the nature of tuberculosis; they form compact bodies of public-spirited citizens, whose influence is of the greatest value in so moulding public sentiment that funds shall be forthcoming to erect and maintain dispensaries, sanatoria, and homes for the consumptive poor. Through their assistance, and that of the public press, we may hope eventually to obtain State and municipal appropriations for the suitable care of the consumptive poor. New York State has made a small beginning in this way, and it is hoped that the State sanatorium, now in course of erection in the Adirondacks, may lead to very much larger appropriations for this purpose. The State is spending many millions of dollars annually for the care of the insane, and while this is absolutely necessary, for humanitarian reasons, I have no hesitation in saying that far greater returns would be obtained from the expenditure 
of one-quarter the amount in the prevention and cure of tubercular disease.

"I believe that tuberculosis may be practically stamped out. The reduction in the mortality from it in New York City, since I886, has been about 40 per cent., which means if applied to the Greater City, a decrease of more than 6000 in the number of deaths annually caused by it. The vast significance of this is still more enhanced when we remember that to a very large extent these deaths take place in the working period, between fifteen and fifty-five years of age. I have no doubt that the measures, first begun in a very small way in New York City fifteen years ago, inadequate as they have been, have resulted in saving the lives of at least twenty thousand persons. The annual deaths in the Greater City still number between nine and ten thousand, and we know that these are, to a very large extent, unnecessary." 


\section{CHAPTER VIII}

\section{LEPROSY}

E EPROSY is an infectious disease due to a bacillus, discovered in 1879 by Hansen, a Norwegian physician. This bacillus is found in great numbers in the cells contained in the leprous nodules, which are characteristic of the disease. In its form the leprosy bacillus resembles the tubercle bacillus, but bacteriologists have failed in their attempts to cultivate it in artificial media and in communicating the disease to lower animals, as has been repeatedly done in the case of the tubercle bacillus. Nor has it been determined in a definite manner how the disease is contracted. It is not, unless in very exceptional cases, communicated by the sick to those in close association with them. Thus among the Sisters of Charity who have for many years taken care of the sick in the lepers' hospital at Havana, not one has ever contracted the disease. Inoculation experiments in healthy men, made with the knowledge and consent 
of those inoculated, have been repeatedly made by investigators with a negative result. In these inoculations, blood, pus, and portions of the leprous tissues containing numerous bacilli have been used. In a single instance only was such an inoculation followed by the development of leprosy, but this man belonged to a leprous family and it is not cer-

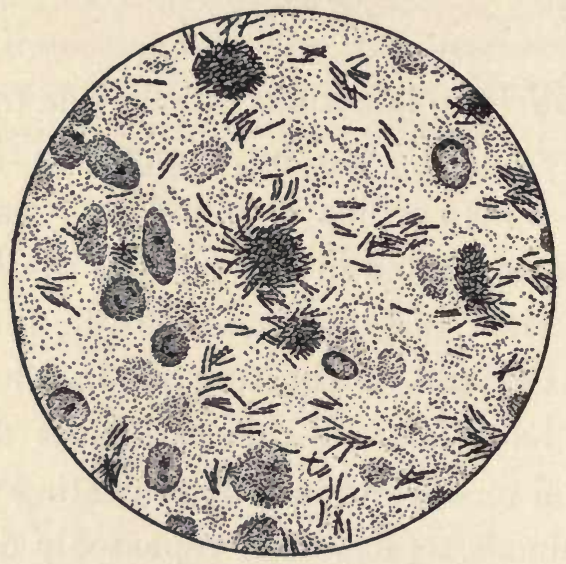

FIG. 6. Bacillus of leprosy, as seen in a thin section of a leprous nodule : magnified rooo diameters.

tain that the disease in his case was a result of the inoculation.

The question as to whether leprosy is contagious has been much discussed by physicians, and while this has been most positively denied by many physicians, and certainly does not occur under ordinary conditions, the opinion seems to be gaining ground 
that in some way it must be communicated from the sick to the well. Indeed, it would otherwise be difficult to conceive as to how the disease is propagated. Certain families manifest a special susceptibility to the disease and its hereditary transmission is claimed by some authorities; but if we admit this it will not account for the development of the disease in previously healthy adults and in the cases occurring in individuals whose parents had no taint of the disease. Moreover, there are numerous instances of individuals born of leprous parents who have grown to manhood or womanhood without developing the disease. The intimate association of husband and wife, also, fails in most instances to give rise to leprosy in the healthy member of the pair, the other being a leper.

Notwithstanding the facts stated most recent authorities insist that leprosy is a contagious disease which can be communicated, under conditions not yet well understood, by those infected with the bacillus of the disease to healthy individuals having a special predisposition to such infection. According to Dr. Morrow of New York, who has made a special study of this disease: "The literature of leprosy abounds with well-authenticated cases of individual contagion, showing in the clearest and most positive manner that the disease spreads from leprous to healthy persons by contact." The same author says : 
"If the observer limits his field of examination and judgment to certain parts of Europe and the United States, he may find little clinical evidence of the active spread of leprosy by contagion. Observation shows that leprous germs introduced into these favoured regions do not take root and spread; they rarely survive the death of the leper. In New York, for example, large numbers of lepers coming from countries where leprosy is endemic have lived for years, many of them have been sent to general hospitals, where they have died, and yet, so far as is known, no case of indigenous leprosy can be traced to association or contact with these patients. The same experience has been noted in London, Paris, and Berlin, where lepers from other countries have flocked for treatment and have been received in the general hospitals without special measures of isolation, and yet no case of leprous contamination has been traced to contact with them."

The same author calls attention to the fact that one hundred and sixty Norwegian leper immigrants have settled in our Northwestern States, where most of them have eventually died, but no cases have occurred among those associated with them. On the other hand, leprosy has extended in Louisiana, in the Sandwich Islands, and in many other parts of the world, as a result, apparently, of the introduction of cases from other endemic foci of infection.

Morrow believes that the main factors which lead to the propagation of the disease are individual predisposition and insanitary surroundings. Certain authors insist that a fish diet has the effect of establishing a predisposition to the disease. In support of 
this theory they call attention to the fact that the disease is more prevalent, in certain countries, along the sea-coast, where fish contributes a considerable proportion of the diet of the inhabitants. The influence of insanitary surroundings and uncleanly habits in promoting the spread of the disease is generally admitted.

The conditions among the peasantry of Norway, where the disease is quite common, are stated by Leloir as follows :

"The Norwegian peasant is very dirty. The greater number of the peasants have never taken a bath. They may sometimes wash (once a week) the hands and face, and the feet once a year, but the other parts of the body are not washed from the day of their birth to that of their death. Their clothing is never taken off even for the purposes of sleeping. It is generally made of wool. Their garments are never washed. Dirt is allowed to accumulate upon them, and when not too rotten, they are often transmitted from generation to generation. They live promiscuously gathered together in a small house. . . . Dung and filth are accumulated around the house amidst pools of dirty water. Often pigs, poultry, and other domestic animals live with the family. Almost always several persons sleep in the same bed, which is nothing but a kind of wooden chest upon which are thrown some sheep skins or goat skins which are scarcely ever washed. If a stranger comes he shares the bed. Everybody eats at the same table, from the same dish, often with a common spoon, and drinks from the same vessel."

With reference to the habits of the natives of the Sandwich Islands Mr. Meyer, Superintendent of the leper sfttlement at Molokai, says: 
"Their modes of eating are so extremely careless that inoculation can readily take place through the mouth, by means of the saliva or otherwise. They pass their pipes from mouth to mouth, whether any of their number is a leper or not; they kiss and rub their noses together; they eat out of the same calabash with their fingers and drink out of the same cup; in eating fish or meat it is not cut up, but one takes the meat in his hand, and, after taking a bite, passes it on. They drink ava, which is prepared by others chewing the root, and whether the one chewing is a leper or not is not considered. Foreigners also become addicted to this habit of ava drinking, and it is remarkable that most of the foreigners who have become lepers are ava drinkers."

These habits would evidently favour the communication of the disease in the manner by which Morrow believes this usually occurs, which is shown by the following quotation from his valuable article in the Twentieth Century Practice of Medicine. He says: "In the vast majority of cases, I believe that the vehicles of the virus through which contagion is effected are the secretions of the nose and mouth, and that the port of entrance is the mucous membrane of the respiratory and intestinal tract, with secondary infection through the blood or lymphatic system."

There is little doubt that leprosy has existed from a remote antiquity, although there is some difficulty in identifying the disease as we know it by the descriptions of leprosy given by Hebrew, Greek, and Arabian authors. In the Middle Ages, also, although the disease was far more prevalent than at the present 
day, other diseases were, no doubt, frequently confounded with it. The disease appears to have existed in Egypt, in India, and probably in other parts of Asia long before it was introduced to the countries of Europe, where it gained its widest prevalence between the sixth and twelfth centuries of our era. Its decline in Europe has, to a great extent, been progressive since the fifteenth century.

In Great Britain, the disease was prevalent during the twelfth, thirteenth, and fourteenth centuries, and numerous "leper houses" existed. The disease was less common during the following centuries, and became practically extinct during the latter part of the eighteenth century, its final seat having been in the Shetland Islands. In 1742 a public thanksgiving was ordered for the permanent disappearance of leprosy from the Shetland Islands. The last leper of the Shetland Islands, it is stated, died in the Edinburgh Infirmary in 1798 (Morrow). In southern Europe the disease began to decline towards the close of the seventeenth century.

At the present day India and China are the chief centres of leprous infection. The census of India for I 891 gives the total number of lepers in that country as 114,239. The disease also prevails in Cochin China, in the Malay Peninsula, in the Dutch East Indies, in the Philippine Islands, and in Japan. In 
the last-mentioned country it was reported, in 1897 , that there were 23,647 lepers, a majority of whom were located in the coast region of the islands. In 1893 the total number of lepers in Egypt was 2204. In Spain, Portugal, France, and Italy a limited number of cases of leprosy may be found, but Great Britain Holland, Belgium, Germany, Austria, Switzerland, and Denmark are practically free from the diseasea few imported cases no doubt exist in each of these countries. In Norway, which for many years has been the principal infected centre in Europe, the number of cases has been constantly diminishing during the past fifty years. In 1856 the number of cases reported was 2870 , in 1895 the number had fallen to 688. The reduction was partly due to the emigration of lepers to other countries (287). In Russia 1200 cases of leprosy were reported as living within the limits of the empire in the year 1895 , when an official census was taken.

In South America and in the West Indies there are a considerable number of lepers, especially along the coast of Brazil and of British Guiana. The disease also exists in the states of Central America and in Mexico.

Cases of leprosy not infrequently arrive in the United States from Norway, from China, or from the Hawaiian Islands, but the policy of the Govern- 
ment and State health authorities now is to return them to their native countries when practicable.

We have in the United States one centre of leprosy infection which has existed for many years, and in which new cases are still developed from time to time. This is in Louisiana. According to Dr. Dyer, who has made a special investigation with reference to leprosy in New Orleans and vicinity, 270 cases have developed in that State since 1878 . Of these, 171 were born in Louisiana and 39 were born in Europe.

What has already been said with reference to the history and geographical distribution of leprosy indicates that even when no special precautions are taken there is little danger that the disease will spread to any extent in countries where the people are accustomed to civilised ways of living and pay a reasonable degree of attention to cleanliness. Nevertheless, most writers upon the subject, and health authorities, national and local, insist upon the isolation of lepers; and, where there are a considerable number of these unfortunates, upon their segregation in suitably located colonies, where they can have whatever comfort and enjoyment is possible under the conditions existing in such isolated localities, while at the same time they are removed from the possibility of communicating the disease to others. I am in full accord with 
this policy. But the unreasonable fear of lepers, which has existed from the earliest times, and is often manifested at the present day, is evidently not justified, in view of the very remote danger of the disease being contracted by any ordinary association with them. The danger from tuberculosis is far greater, and this disease claims nearly 150,000 victims annually within the limits of the United States, yet no one proposes the isolation of cases of pulmonary tuberculosis, and indeed this is not necessary for the prevention of the disease if the complete disinfection of all infectious material can be secured-that is, of the material expectorated by those suffering from the disease. In leprosy it will, of course, be advisable to destroy all pus, saliva, etc., which contains the bacillus, but we can scarcely insist upon the rigid measures of disinfection which are essential for the prevention of diphtheria, or smallpox, in view of the fact that painstaking researches fail to show that the disease, under ordinary conditions as they exist to-day, is communicated to healthy individuals who are associated in an intimate way with the sick. 


\section{CHAPTER IX}

\section{DIPHTHERIA}

$\mathrm{D}^{1+4}$

IPHTHERIA, like tuberculosis, is a disease which is propagated principally through the medium of the sputa of infected individuals, which contains in large numbers the specific bacillus to which the disease is due. This bacillus effects a lodgment in the fauces, or in the posterior nasal passages of susceptible individuals and invades the mucous membrane, causing a localised inflammation and a fibrinous exudation - so-called "false membrane." Its first appearance is very commonly upon the tonsils. As the disease progresses symptoms of poisoning by the deadly toxin of the diphtheria bacillus are developed.

The disease is more frequent and more fatal in young children than in adults. The mortality is greatest in infants and comparatively low in children over twelve years of age. The disease differs greatly as to its malignancy during different epidemics. 
There are evidently many different breeds of the diphtheria bacillus, and its pathogenic virulence is increased or diminished as a result of conditions relating to its growth. A series of cases in very susceptible individuals is favourable to an increase in the virulence of the germ. On the other hand, when obtained from the throat of one who is comparatively immune, or cultivated in artificial media outside of the body, it is less virulent.

While the most robust children are subject to attack, delicate and poorly nourished children are more susceptible and more likely to die. A chronic or acute catarrhal inflammation of the throat or nasal passages predisposes to infection.

The bacillus of diphtheria was first described by the German pathologist, Klebs, in $188_{3}$, and the following year the fact that it is the specific cause of this disease was demonstrated by Löffler, a surgeon in the German army and a pioneer in bacteriological researches. Hence it is often spoken of as "the Klebs-Löffler bacillus." Cultures of this bacillus are very pathogenic for guinea-pigs, rabbits, chickens, pigeons, and cats, and to a less extent for dogs, horses, and cattle. Rats and mice have a natural immunity. The immunity of certain individuals is shown by the fact that when associated with diphtheria patients they may carry virulent diphtheria 
bacilli in their throats without manifesting any symptoms of the disease. Non-virulent varieties of the diphtheria bacillus are not infrequently found in the throats of healthy persons. Much attention has been given by bacteriologists to the question as to how long the bacillus may persist in the throats of convalescents from the disease. Drs. Park and Beebe,

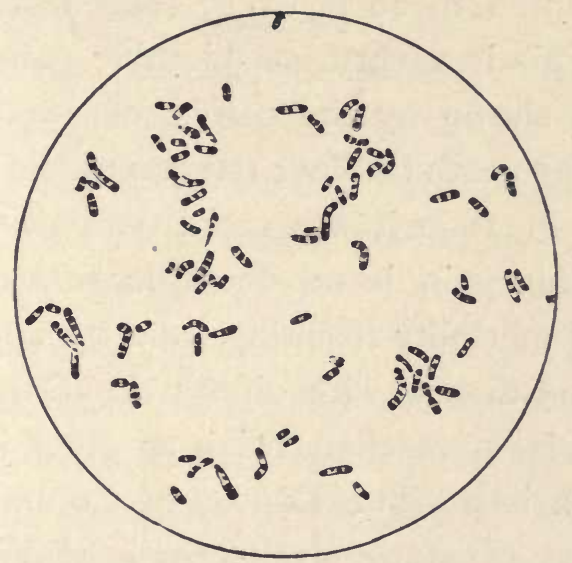

FIG. 7. Bacillus of diphtheria ; magnified 1000 diameters.

of the New York Health Department, found in a series of 605 cases that the bacilli disappeared in three days in 304, in seven days in 176 , in twelve days in 64, in fifteen days in 36 , in three weeks in 12 , in four weeks in 4, and in nine weeks in 2. This shows the necessity for expert opinion before one who has recovered from the disease is allowed to associate with susceptible children. 
It is now recognised by physicians that "membranous croup" is due to the diphtheria bacillus, and is in fact a form of diphtheria, most common in very young children, in which the larynx and larger bronchial tubes are the seat of infection.

The total number of deaths reported from diphtheria in the last census year ( 1900 ) was 28,959 . This amounted to 29 in every 1000 deaths from all causes. This is a very considerable gain upon the proportion shown by the census of 1890 (49.7 per 1000). The death-rate per 100,000 of the population was 45.2 ; in 1890 it was more than twice as great (97.8). This gain is no doubt largely due to the diminished mortality resulting from the specific treatment of the disease with diphtheria antitoxin. The death-rate is more than twice as great in cities as in rural districts. The District of Columbia has the highest rate (75.4); and the State of Vermont the lowest (18.3). It is evident that, in view of our previous statement that the mortality is greater in cities than in rural districts, it is not fair to compare the District of Columbia with States having a large rural population. As compared, however, with the average rate for cities $(52.8)$ the record of the capital city of the nation is not creditable. It is due to the large coloured population of the city and the comparatively large death-rate among negroes as 
compared to whites. This applies only to children under one year of age $(345.7$ per 100,000 coloured and I54.9 per 100,000 white); beyond this age the mortality is somewhat greater among white children.

For the prevention of diphtheria it will not only be necessary to disinfect the sputa of the sick and all articles liable to be soiled by it, but also to isolate infected individuals until all danger of their communicating the disease has passed. The measures recommended in the previous chapter for the disinfection of tuberculous sputum apply equally well for diphtheria. The diphtheria bacillus is promptly destroyed by a temperature of $140^{\circ}$ Fahr. ( $60^{\circ}$ C.) and by the various germicides heretofore mentioned. Boiling water, for all articles which can be sent to the laundry and for the disinfection of sputa in metal or porcelain receptacles, is the cheapest and most reliable disinfectant, but it will usually be necessary to use a solution of carbolic acid (three to five per cent.), or one of the other coal-tar products (lysol, creolin, tricresol), in the sick-room. Woollen clothing and other articles which would be injured by immersion in boiling water may be disinfected by steam or by formaldehyd gas.

The New York Health Department makes the following judicious recommendations with reference to the prevention of diphtheria: 
"If possible, one attendant should take entire care of the sick person, and no one else besides the physician should be allowed to enter the sick-room. The attendant should have no communication with the rest of the family. The members of the family should not receive or make visits during the illness.

"The discharges from the nose and throat must be received on handkerchiefs or cloths, which should be at once immersed in a carbolic solution (made by dissolving six ounces of pure carbolic acid in one gallon of hot water, which may be diluted with an equal quantity of water). All handkerchiefs, cloths, towels, napkins, bed linen, personal clothing, night-clothing, etc., that have come in contact in any way with the sick person, after use should be immediately immersed, without removal from the room, in the above solution. These should be soaked for two or three hours, and then boiled in water or soapsuds for one hour.

"In diphtheria and scarlet-fever, great care should be taken in making applications to the throat or nose, that the discharges from them in the act of coughing are not thrown into the face or on the clothing of the person making the applications, as in this way the disease is likely to be caught.

"The hands of the attendant should always be thoroughly disinfected by washing in the carbolic solution, and then in soapsuds, after making applications to the throat or nose, and before eating.

"Surfaces of any kind soiled by the discharges should be immediately flooded with the carbolic solution.

"Plates, cups, glasses, knives, forks, spoons, etc. used by the sick person for eating and drinking must be kept for his especial use, and under no circumstances removed from the room or mixed with similar utensils used by others, but must be washed in the room in the carbolic solution and then in hot soapsuds. After use the soapsuds should be thrown into the water-closet and the vessel which contained it should be washed in the carbolic solution.

"The room occupied by the sick person should be thoroughly aired several times daily, and swept frequently" (we think it better to wipe floors with cloths wet with a disinfecting solution), 
"after scattering wet newspapers, sawdust, or tea-leaves on the floor to prevent the dust from rising. After sweeping, the dust upon the woodwork and furniture should be removed with damp cloths. The sweepings should be burned and the cloths soaked in the carbolic solution. In cold weather the sick person should be protected from draughts of air by a sheet or blankets thrown over his bed while the room is being aired.

"When the contagious nature of the disease is recognised within a short time after the beginning of the illness, after the approval of the Health Department Inspector, it is advised that all articles of furniture not necessary for immediate use in the care of the sick person, especially upholstered furniture, carpets, and curtains, should be removed from the sick-room.

"When the patient has recovered from any one of these diseases the entire body should be bathed and the hair washed with hot soapsuds, and the patient should be dressed in clean clothes (which have not been in the room during the sickness) and removed from the room. 'Then the Health Department should be immediately notified, and disinfectors will be sent to disinfect the room, bedding, clothing, etc., and under no conditions should it be again entered or occupied until it has been thoroughly disinfected. Nothing used in the room during the sickness should be removed until this has been done."

Where the parents have not ample means to provide for the services of a trained attendant, and a suitable room for the isolation of the patient, it will be much better for the sick person, and for all concerned, to send the patient to a contagious-disease hospital, when this is practicable. The diphtheria bacillus resists desiccation for a long time and rooms or clothing soiled with material containing this deadly disease germ remain dangerous for months if they have not been properly disinfected. 
The diphtheria antitoxin which is now extensively and successfully used in the specific treatment of the disease may also be used to prevent its development in persons exposed to infection. From five to ten cubic centimetres of a reliable antitoxin is used for this purpose. This is injected subcutaneously and is a harmless procedure which has been proved to be efficacious in most cases, or at least to greatly modify the severity if an attack occurs. Where children have been exposed to infection, or under circumstances which prevent the proper isolation of a diphtheria patient, it is prudent to resort to such preventive inoculations. As is generally known, the antitoxin is usually obtained for practical purposes from horses which have been rendered immune by repeated and gradually increasing doses of diphtheria toxin-that is, the poisonous substance developed by the diphtheria bacillus during its growth in suitable culture media. The antitoxin is contained in the blood of the immune animal, and the clear blood serum which separates on standing, from the "clot," which contains the red and white blood corpuscles, is spoken of as "antitoxin" and is used in the treatment and prevention of the disease. From this blood serum a more concentrated antitoxin may be obtained by chemical methods, but this has not come into practical use. 


\section{CHAPTER $\mathrm{X}$}

\section{INFLUENZA}

EPIDEMIC influenza ("la grippe") is an infectious disease due to a minute bacillus discovered by the German bacteriologist Pfeiffer in 1892 . This bacillus is found in great numbers in the purulent bronchial secretion coughed up by persons suffering from influenza and the disease is propagated by contact with the sick and with articles infected by them, just as is the case in diphtheria, or whooping-cough. The rapid extension and wide prevalence of the disease is due to the facts that persons of all ages are susceptible, and that isolation of the sick and disinfection of sputa are rarely practised, on account of its comparatively mild character. Nevertheless, the disease is attended with considerable danger, especially for old persons or those in feeble health; and it is the direct or remote cause of many deaths. The official records of Prussia for $189^{2}$ show a mortality from this disease of $15,9 \mathrm{I}$, of whom over half were more 
than sixty years of age. The total number of deaths in the United States during the census year I900 was 16,645 . As in Germany and elsewhere a large proportion of the deaths occurred in persons over sixty years of age or among young children, under five. But these figures by no means represent the total mortality from the disease. Many deaths re-

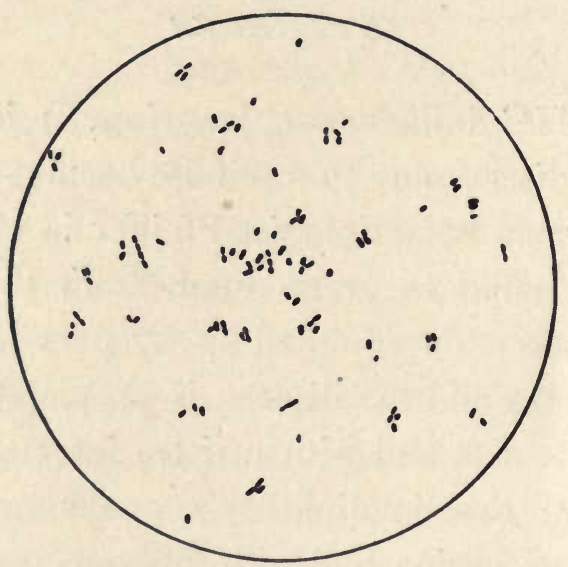

FIG. 8. Bacillus of influenza ; magnified 1000 diameters.

corded as due to pneumonia are the result of a preceding attack of influenza. Other complications or results of the disease which may have a fatal termination are connected with the brain and spinal cord, the heart or the kidneys. Serious eye and ear troubles, also, not infrequently follow an attack of influenza.

Wide-spread epidemics of influenza have prevailed in Europe from an early period. It prevailed in Italy, 
Germany, and England as long ago as 1173 , and since that time there have been repeated epidemics. The epidemic which started in the year 1510 was the first one occurring in the British Islands of which we have authentic and accurate accounts. This epidemic had such a wide distribution that it is said to have "raged all over Europe, not missing a family and scarce a person" (Thomas Short).

Another still greater epidemic, or "pandemic," dates from the year 1580 . We cannot spare the space for an historical account of the numerous epidemics which occurred during the seventeenth and eighteenth centuries, but will pass on to the nineteenth.

In 1802 the disease made its appearance in France and during the following year obtained wide extension in European countries. In 1807 it was generally disseminated in North America. It again prevailed both in North and South America in 1815 and 1816; the next epidemic in the United States was inaugurated in 1824 and lasted for several years. The epidemic of 1833 was apparently confined to the Eastern Hemisphere, where the countries of Europe, Asia, and Northern Africa were invaded. It is said that in St. Petersburg " not one person escaped the influenza" during this epidemic. The next extensive epidemic in Europe was during the years 1836-37; 
and again the Eastern Hemisphere was visited in I847-48. In the winter of $185 \mathrm{I}$ the disease obtained wide prevalence both in North and South America. In 1857-58 it again prevailed extensively in both hemispheres and in some localities (Rome and Naples) was attended with a high rate of mortality. During the winter of $1874-75$ it again prevailed extensively in Europe and America. During the epidemic of I 889-90 the disease obtained, within a brief period, a wider extension in all parts of the world than in any previous epidemic. This pandemic apparently had its origin in Central Asia. The United States has not been free from the disease since the date last mentioned, and some very susceptible individuals, especially in cities, suffer an attack almost annually.

The incubation period is comparatively brief, being from two to six days. Those whose occupation keeps them out-of-doors during the daytime are less subject to attack than factory hands or others, who are more exposed to contagion by reason of their living in closed rooms containing numerous occupants. The disease prevails to some extent throughout the year but the largest number of cases occur during the winter and spring months. Catarrhal conditions of the bronchial and nasal mucous membranes, and the meteorological conditions which are favourable to "catching cold" constitute predisposing causes. Per- 
sons who live in over-heated and ill-ventilated apartments are also especially subject to attack. The contagious nature of the disease is shown by the fact that an initial case in a household, a school, a factory, or a prison commonly leads to a local epidemic which may include all of those exposed to infection.

The prevention of the disease under existing conditions seems impracticable, as isolation of the sick, and especially of mild cases and of convalescents who are still capable of communicating the disease, is a measure which is not likely to be considered with favour by the public and has not been seriously proposed by health authorities. Those who are in delicate health, and especially elderly persons, should endeavour to avoid contact with persons suffering from influenza. And those suffering from the disease should make it a matter of conscience not to communicate it to their friends by untimely visits, by kissing, etc. For one who recognises the contagious nature of the malady and who has had some previous personal experience as to the discomfort attending an attack, it is not pleasant to have a friend call and announce the fact that he, or she, is " just recovering from an attack of influenza," or is "really too sick to be out, but could not resist the temptation of running over to see you and to inquire about the children"-and perhaps bestows a kiss upon the 
" little dears." Those who are confined to the house by an attack should by all means spit in a metal or porcelain receptacle containing a disinfecting solution, and other members of the household should, as far as is practicable, be kept from their room. Those who are able to go about should have an ample provision of pocket-handkerchiefs and these should be immersed in a disinfecting solution upon their return to the house.

Fortunately the influenza bacillus is soon killed by desiccation and by exposure to sunlight. An effectual way of disinfecting woollen clothing, stuffed furniture, etc., will therefore be to expose it in the open air. The bacilli in dried sputum do not survive more than twenty-four hours and a majority of them are killed in seven or eight hours. Diffuse daylight also hastens their death. It is therefore unnecessary to resort to the rigid measures of disinfection recommended in diphtheria and tuberculosis, in which diseases the specific germs resist desiccation for a long time. Free ventilation for two or three days and exposure of infected articles in the open air will ensure the disinfection of the room and its contents. The disease is no doubt largely spread by the minute droplets ejected by the sick in coughing or sneezing, each one of which contains thousands of influenza bacilli. 


\section{CHAPTER XI}

\section{PNEUMONIA}

L OBAR or "croupous" pneumonia is due to a micrococcus discovered by the present writer in I 880 and since demonstrated to be the cause of this infectious disease. The micrococcus of pneumonia is found in great numbers in the exudate which fills the air cells of the portion of the lung involved (one or more lobes) and in the sticky sputum coughed up by the patient. This is "rusty" in appearance on account of the presence of red blood-corpuscles. In fatal cases of pneumonia the micrococcus is not infrequently found, also, in the blood of the infected individual, but as a rule the disease may be regarded as a localised infection. The symptoms are due in part to a deficient supply of oxygen from the occlusion of the air cells in one or more lobes and in part to the absorption of the toxin produced by the micrococcus. The fatality of the disease depends largely upon the extent of lung tissue involved and also upon the age 
and vital resisting power of the individual attacked. Depressing influences of all kinds predispose to an attack, and especially alcoholism, malnutrition, and insanitary surroundings. Pneumonia also frequently occurs as a complication of other infectious diseasesmeasles, influenza, typhoid fever.

It is most prevalent during the spring months. In

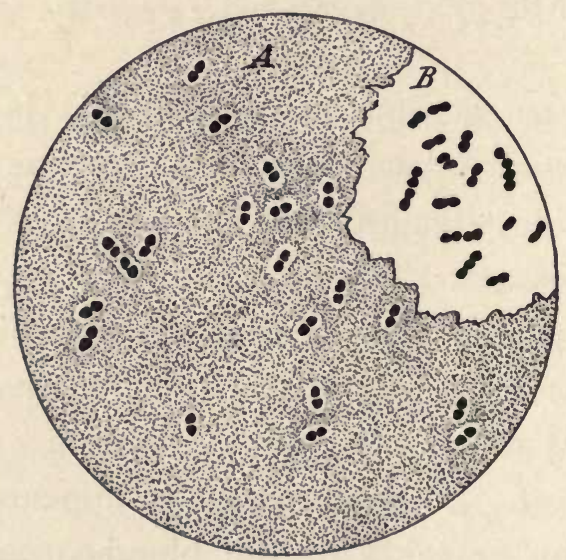

Fig. 9. Micrococcus of pneumonia; magnified 1000 diameters. $A$, with "capsule," as seen in the blood of an infected animal; $B$, as seen in a culture fluid.

the United States the area of greatest prevalence is the Middle Atlantic Coast region. The Gulf Coast region and the South Atlantic Coast region have the smallest proportion of deaths from this disease. The North Atlantic Coast region, the Interior Plateau, the Central Appalachian region and the region of the Great Northern Lakes have all about the same rate ( 103 to 106 
per 1000 deaths from all causes). The greatest mortality occurs in children under five years of age ( 388.6 out of 1000 deaths from this disease).

The total number of deaths reported as due to pneumonia during the census year 1900 was 105,971 , the proportion in 1000 deaths from all causes being 106.I. This is an increase over the ratio shown by the preceding census (90.6).

The State of New York gave the highest rate (228.4) and Michigan the lowest (109.3). The rate was considerably higher in cities than in the rural districts, and the census of 1900 shows an increase in the cities and a diminished rate in the country over the rates of 1890 . The death-rate among the coloured population (349 per 1000 deaths) was considerably greater than among whites (184.8). It was also larger for foreign-born whites than for native whites. The death-rate from pneumonia per 100,000 of the population was 190 in 1900 and 186.9 in 1890 . These figures show that pneumonia ranks with tuberculosis as a cause of mortality in the United States and that while the number of deaths from the lastmentioned disease is diminishing there has been a decided increase in the number of deaths from pneumonia during the past ten years.

The recent statistics of the city of Chicago show a notable increase in the number of deaths from this 
disease. In the Bulletin of the Health Department of that city for the month of May, I903, the following statement is made:

"Since the first of January there have been $289 \mathrm{r}$ deaths from pneumonia, as compared with $13^{2} \mathbf{I}$ from consumption and 1238 from all other communicable, contagious, or infectious diseases, including diphtheria, erysipelas, influenza, measles, puerperal fever, scarlet fever, smallpox, typhoid fever, and whooping-cough. This is an excess of $3^{82}$ pneumonia deaths over the deaths from all the other preventable diseases- 570 , or I 8.8 per cent. more than the deaths from consumption, and 1653 , or 133.5 per cent. more than those from the other specified diseases."

The micrococcus of pneumonia, unlike the tubercle bacillus, is able to live in the mouths of healthy persons, where it finds the necessary pabulum for its multiplication in the normal salivary secretions. While pneumonia may prevail as an epidemic, as a result of the transmission of the disease from the sick to those associated with them, it is well known that many solitary cases occur, in families, which cannot be traced to preceding cases and in which the disease does not spread, although no precautions have been taken in the way of isolating the patient or disinfecting his sputa. The infection in such cases probably results from the presence of the micrococcus in the salivary secretions of an individual whose lungs, as a result of "catching cold" or of an attack of measles or of influenza, have become vulnerable, - that is, are sus- 
ceptible to infection because of a localised congestion or inflammation of the mucous membrane, or a lowered vitality of the tissues due to some of the causes previously mentioned (alcoholism, crowd-poisoning, etc). My own researches and those of other investigators show that this micrococcus is present in the salivary secretions of a considerable proportion of healthy persons, both in this country and in Europe. Also, that it varies greatly in pathogenic virulence as obtained from this source and from the sputa of patients with pneumonia.

Epidemics of pneumonia, in prisons, and on shipboard, have occurred with sufficient frequency to show that the disease may be communicated by the sick to those associated with them. That this does not occur more frequently is probably due to the fact that patients with pneumonia are confined to bed and that the abundant expectoration, among decent people, could scarcely fail to be deposited in a receptacle of some kind, or to be wiped from the mouths by handkerchiefs or cloths which are destroyed by fire or sent to the laundry. Also to the fact that the micrococcus of pneumonia has far less resisting power to desiccation than the tubercle bacillus or the diphtheria bacillus and would be less likely to survive in dust. The tenacious sticky nature of the sputa also prevents, to a great extent, the coughing up of minute droplets, 
which in tuberculosis and in influenza play an important part in the propagation of the disease. The micrococcus of pneumonia may, however, retain its vitality for a considerable time in dried masses of sputum. It is destroyed by exposure for a few hours to direct sunlight, and by a temperature of $140^{\circ} \mathrm{Fahr}$. within a few minutes; also by all germicidal agents in comparatively small proportion. The directions already given for the disinfection of the sputa of patients suffering from tuberculosis or diphtheria apply as well to the material expectorated by patients with pneumonia, and this should never be neglected.

The prevention of pneumonia will, to a large extent, depend upon the avoidance by individuals of the predisposing and exciting causes of the disease. Proper food, proper exercise, dress suitable to the climate and season, avoidance of all excesses, and especially of the habitual use of alcoholic drinks, will all tend to preserve the individual from infection by the micrococcus of pneumonia. On the other hand insufficient food, insufficient clothing, alcoholic excesses, insanitary surroundings, and a recent attack of certain other infectious diseases (measles, influenza, typhoid fever) are potent predisposing causes. Exposure to cold, to draughts, or wet feet may, in conjunction with the presence of the specific micrococcus, become the direct exciting cause of an attack. 


\section{CHAPTER XII}

WHOOPING-COUGH

$W^{\text {HOOPING-COUGH is another infectious dis- }}$ ease in which the germ is no doubt present in the secretions from the mucous membrane of the respiratory passages, although this germ has not yet been demonstrated to the entire satisfaction of the medical profession. The disease is propagated by contact or close association with the sick, but the infection does not persist in the sick-room or attached to the clothing of the sick, as is the case in diphtheria, measles, and scarlet fever. Probably the germ quickly perishes outside of the bodies of the susceptible individuals who serve to propagate it. It has been noted that the disease is rarely conveyed by a third person and, when the clothing of those in attendance on the sick is changed, the danger of its being transmitted in this way is very slight. But susceptible children contract the disease after very brief contact with one suffering from it. The infection 
can be conveyed from the very outset of the attack, and probably in protracted cases for several monthsjust how long has not been definitely determined. The susceptibility to the disease among children is very great, but diminishes with advancing age. Adults and even old persons, however, occasionally suffer an attack. The immunity resulting from a single attack is very great.

The number of deaths reported from whoopingcough, in the United States, during the census year I 900 was 9958 . The death-rate per 100,000 of the population was I2.7. The comparatively non-fatal character of the disease is shown by these figures, as it is one of the commonest and most widely spread of infectious diseases. A considerable number of deaths are, however, indirectly due to an attack of this disease as it is recognised as one of the predisposing causes of tuberculosis. The death-rate in the United States is more than twice as great among coloured children as among whites. There has been a considerable decrease in the mortality from whoopingcough during the ten years ending in 1900. The census returns show that the greatest mortality occurs among infants, less than one year of age. Beyond the age of five the mortality is very slight. The months of greatest mortality are March, April, and August; of lowest, October and November. 
In the prevention of this disease isolation of the sick must be given the first place. Children and especially young children should be carefully guarded from exposure to infection. The mucus coughed up by the sick should be received upon handkerchiefs or squares of muslin and these should be placed in a disinfecting solution or burned. Free ventilation of the apartments occupied by the sick and prolonged exposure of infected articles to the sun and air will suffice for the disinfection of the sick-room and its contents. Isolation of the patient should be continued so long as the characteristic paroxysms of coughing continue and for a few days after they cease. The recurrence of a spasmodic cough after this time, if two or three months has elapsed since the outset of the attack, does not call for renewed isolation of the patient, as such spasmodic coughing is not unusual after all danger of infection has passed. 


\section{CHAPTER XIII}

\section{SMALLPOX}

$H^{A V I N G}$ given some account of the causes and prevention of the "filth diseases" and of the diseases which are communicated through the medium of material coughed up from the lungs or upper airpassages of infected individuals, I shall now briefly consider that group of infectious diseases known under the general name of "eruptive fevers," and first in importance comes smallpox. This disease, which was formerly a scourge of the human race, has been largely robbed of its terrors by vaccination.

There is reason to believe that smallpox has prevailed in India and China from a remote antiquity. It was probably introduced into the British Islands about the year I 24I. From the end of the thirteenth century until the general adoption of vaccination as a preventive measure smallpox ranked as the most destructive of the pestilential diseases. Lord Macaulay describes the ravages of this disease in prevaccination times as follows : 
"That disease, over which science has since achieved a series of glorious and beneficent victories, was then the most terrible of all the ministers of death. The havoc of the plague had been far more rapid; but the plague had visited our shores only once or twice within living memory; and the smallpox was always present, filling the churchyards with corpses, tormenting with constant fears all whom it had not stricken, leaving on those whose lives it spared the hideous traces of its power,-turning the babe into a changeling at whom the mother shuddered and making the eyes and cheeks of the betrothed maiden objects of horror to the lover."

In England and Wales the annual mortality from smallpox prior to the year 1796 was about 3000 for every million of the inhabitants. Less than a century later, in 1890 , the total number of deaths from this disease during an entire year was only fifteen. In Germany, where vaccination is compulsory and children must be revaccinated at the age of twelve, smallpox is almost unknown.

In the year 1888 there were but I Io deaths from this disease in the entire German Empire. In France, where vaccination is very much neglected, the mortality from smallpox is about 35 per 100,000 inhabitants (1889). Brouardel, a French author, from a consideration of the statistics of the two countries, arrives at the conclusion that "revaccination ought to be made obligatory." In Scotland, when vaccination was optional (1855-64), the mortality was considerable ( 340 per million inhabitants), but when it 
was made compulsory the number of deaths fell to 80 per million. In Sweden the mortality in the eighteenth century exceeded 2000 per million of the inhabitants. From I80I to I8I5, when vaccination was largely practised but was not compulsory, the deathrate fell to 631 per million. Since this preventive measure has been made compulsory the rate has continued to fall until it is not more than 8 per million.

The immunity resulting from vaccination is not absolute and it is well known that very susceptible persons may suffer a second or even a third attack of smallpox. But attacks in vaccinated persons are comparatively rare and comparatively mild.

This is shown by statistics collected by the London Board of Health, which show that the mortality among unvaccinated persons was $20.85 \%$, while among the vaccinated it was only $3.40 \%$. The gradual loss of immunity after vaccination is established by the well-known fact that revaccination after a longer or shorter interval is successful in a considerable proportion of the cases. This is therefore to be recommended whenever smallpox is prevalent.

As a result of neglect of vaccination an epidemic occurred in Sheffield, England, some years ago. Statistics collected during this epidemic (1987-88) show that among children less than ten years of age the vaccinated were attacked in the proportion of 
5 per thousand, and the unvaccinated in the proportion of IOI per thousand. The death-rate among the vaccinated was about one-tenth of one per cent. and among the unvaccinated 44 per cent. Similar and even more favourable statistics could be presented from other parts of England and from all countries where vaccination is systematically practised.

The total number of deaths reported from smallpox, in the United States, during the last census year was 3484 . If the mortality had corresponded with that of England and Wales before the introduction of vaccination it would have amounted to more than $210,000$.

The practical stamping out of smallpox in the District of Holguin, Cuba, and in the island of Porto Rico, since the Spanish-American War, by the vaccination of all the inhabitants, under the direction of the military authorities of the United States, is a matter of record in my annual reports as SurgeonGeneral of the Army for the years 1899 and 1900. In my report for 1899 I say:

"It is understood that the occurrence of smallpox among our troops in the Philippines gave rise in England, where the protective influence of vaccination was under discussion at the time, to the claim that, as vaccination was compulsory in the United States Army, and carried out under military rules, the presence of the disease among our soldiers showed the inefficiency of the process. This claim of the opponents of vaccination is not well 
taken. On the contrary, the history of vaccination and of smallpox in the United States Army suffices in itself to demonstrate that protection from the disease is proportioned to the care with which vaccination is performed. . . Although this disease has prevailed in many parts of the United States during the past fifteen years ( $188_{3}$ to 1897 ), and frequently with epidemic violence, among the civil population in the immediate vicinity of military posts, there occurred only 20 scattered cases, of which 4 were fatal, in a mean strength of 25,000 men."

The cases which occur in various parts of the country, from time to time, are for the most part among unvaccinated individuals. For example, in the city of Chicago, the Bulletin of the Health Department for the month ending May 31, 1903, says :

"During the month forty-five cases of smallpox were discovered and removed to the Isolation Hospital. Of these thirty-nine never had been vaccinated ; six had old, imperfect marks, said to be from vaccination in childhood. Fifteen were unvaccinated children under six years of age."

It is unnecessary to give any further facts in support of the protective value of vaccination, which, since the discovery of Jenner in 1796 , has been established by unimpeachable statistical data in all parts of the civilised world. But there are certain persons whose minds are not penetrated by the logic of facts, or who have not taken the pains to make themselves familiar with these facts, who still oppose vaccination on the ground that it has no value as a preventive measure. 
Recent researches seem to prove that the smallpox germ multiplies in the epithelial cells of that portion of the skin which is involved in the pustules which are characteristic of the disease. This germ is extremely minute and it has not been cultivated in artificial media. It does not belong to the same class as the germs of typhoid fever, cholera, diphtheria, etc. (the bacteria), but to the protozoa.

It is generally recognised that smallpox patients must be isolated and cared for by immune attendants, and that clothing and all articles exposed to infection must be thoroughly disinfected. This is especially important, as the infectious agent or germ is given off from the general surface of the body, attached to epithelium, pus cells, etc. This infectious agent may retain its capacity for harm (vitality) for months in spite of desiccation. An instance is given by Dr. Buck, of New York, in which an unvaccinated infant, when two months. old, contracted smallpox in the room in which it was born and in which a case of smallpox had occurred two years previously.

The danger of infection from a patient before the eruption has developed is very slight if any and it is chiefly through pus and scabs which are formed at a later stage of the disease that the malady is propagated. The time which elapses after exposure before the first symptoms of the disease are manifested is 
usually twelve days and the duration of the disease in cases ending in recovery is about three weeks. 'That the smallpox virus may be carried a considerable distance through the air by currents of wind, and the disease thus be propagated, seems to be well established by the observations of certain English authorities upon the subject. But it is not certain whether it is directly, as dust carried by the wind, or indirectly, attached to the feet of flies which have been in contact with the pustules on the body of a smallpox patient, that the disease has been conveyed in the instances which have been recorded. The extreme limit to which the disease is likely to be communicated in this way probably does not exceed a mile.

The disinfection of the room occupied by a smallpox patient, and all its contents, calls for the most careful, intelligent, and thorough measures. Only absolutely necessary articles should be left in the room and it is well to have screens in the windows to exclude flies. Abundant ventilation is important. While occupied by the patient, floors, window-ledges, and all places where dust is liable to accumulate should be frequently wiped with a cloth wet with a suitable disinfecting solution ( $5 \%$ solution of carbolic acid or I : rooo solution of corrosive sublimate). All washable articles which have been in contact with the sick person or in use in the sick-room should be immersed in 
a disinfecting solution in the room or in an adjoining room. Subsequently they are to be subjected to the action of steam or of boiling water. Woollen clothing, carpets, curtains, etc., are best disinfected by steam. But the room and its contents, after the patient has left it, may be disinfected with formaldehyd gas or by fumigation with burning sulphur. This disinfection should, however, be attended to by an expert and is to be followed by thorough washing of all surfaces with a disinfecting solution, and subsequent scrubbing with hot soap-suds, whitewashing of plastered walls, free ventilation, and exposure of clothing, bedding, etc., in the open air to direct sunlight.

With reference to vaccination, which is our chief reliance for preventing the spread of the disease, we recommend the vaccination of infants and revaccination at the age of ten or twelve, as practised in Germany. After this tests of the immunity of persons of any age should be made, by revaccination, whenever smallpox is prevalent, or when the individual is about to travel in countries where this preventive measure is neglected or carried out in an indifferent manner.

Chicken-pox is a highly contagious disease which fortunately is mild in character and does not contribute to our mortality statistics. The fact that mild cases of smallpox have not infrequently been mistaken for 
varicella, or chicken-pox, should be borne in mind. This mistake has been responsible for the spread of smallpox in numerous instances and is to be guarded against by the isolation of the sick and the measures of disinfection heretofore recommended for preventing the extension of that disease. The period of incubation in chicken-pox is fourteen days. The germ of this disease has not been discovered, but it has been demonstrated that it is present in the "lymph" contained in the vesicles which are characteristic of the disease. 


\section{CHAPTER XIV}

\section{SCARLET FEVER}

SCARLET fever is widely prevalent in the countries of Europe and in North and South America, but is scarcely known in Asia and in Africa. It is said to occur occasionally in China, but to be unknown in Japan. It is impossible to say how long it has prevailed in Europe, but the first to clearly recognise it as a distinct specific disease was the famous English physician Sydenham (1685). It was not introduced to North America until about the year 1735 and first appeared in South America about I 830 .

The germ of scarlet fever has not been demonstrated but, as is well-known, the scarlet fever patient gives off from the surface of his body infectious material by means of which the disease may be communicated, either directly or indirectly, to other susceptible individuals. This consists essentially of cast-off ("desquamated") epithelium, to which the 
infectious agent, or germ, is attached, and which may retain its infecting power for many months. The sputa of scarlet fever patients also contains the germ and by some physicians is believed to be to a large extent responsible for the spread of the disease. As the mucous membrane of the mouth and throat is involved in the eruption which is characteristic of the disease and is in fact the locality where this eruption, followed by desquamation of the epithelium, may first be observed, it is evident that all expectorated material must contain the infectious agent. The disease may be communicated by the scarlet fever patient at any time during the period of desquamation, which may last for a month, or more. The period during which the patient should be isolated, dating from the outset of the attack, is generally fixed at six weeks. The infection may persist in clothing and bedding, in use during the period of desquamation, or exposed in the sick-room, for a year or more.

Certain persons seem to have a natural immunity to scarlet fever and escape the disease although repeatedly exposed to it. In some instances, no doubt, this failure to contract the disease is due to a previous mild and unrecognised attack rather than to an inherited immunity. The protection afforded by an attack of the disease, however mild, is almost abso- 
lute. That is, second attacks are extremely rare, although not unknown.

The fact that adults rarely contract the disease is to a large extent due to the protection afforded by an attack during infancy or childhood. That adults may suffer fatal attacks of the disease is amply proved by the mortality statistics of this and other countries. The greatest number of cases, however, occur between the ages of two and ten years.

In the United States the mortality from this disease during the census year 1900 (6333) was somewhat less in proportion to the population than during the previous census year. The proportion to 1000 deaths from all causes was 6.3 in 1900 and 7.1 in 1890 . These rates are considerably below the rates in England and Wales, where during the year 1899 scarlet fever caused II.7 in every 1000 deaths. The mortality rate is higher in cities than in the country. The States of New Jersey and Massachusetts show the highest mortality rate and the State of Vermont the lowest (this applies only to the registration States, viz. : Connecticut, District of Columbia, Maine, Massachusetts, Michigan, New Hampshire, New Jersey, Rhode Island, New York, Vermont).

Scarlet fever is a disease in which the evil influences of overcrowding and insanitary surroundings 
are not so apparent as in many other diseases. Indeed some authors assert that the children of the rich suffer even in greater proportion than those of the poor. In the United States the mortality among the coloured population is considerably less than among the whites-I 2 deaths in 1000 from all causes among whites and 2.6 per 1000 among coloured. More deaths occur during the winter than during the summer months. This is no doubt partly due to the unfavourable influence of exposure to cold in the development of some of the most fatal complications which are likely to occur during the progress of the disease-nephritis, pneumonia.

For the prevention of scarlet fever we must depend entirely upon isolation of the sick and the disinfection of all infectious material, inasmuch as no method of protecting by inoculation is known. The general directions given with reference to the disinfection of the sick-room and its contents on page 220 apply equally to scarlet fever. Special care must be taken with reference to the sputa of the patient, which should be disinfected by the same methods recommended for tuberculosis (p. I 7I) and other diseases in which the infectious agent is present in secretions from the mucous membrane of some portion of the respiratory tract.

The advantages attending the removal of scarlet 
fever patients to special "contagious-disease hospitals" have been amply demonstrated. Dr. Hope, Medical Officer of Health for the city of Liverpool, refers to this in his report for the year I90I, as follows :

"Want of hospital accommodation goes far to explain the increase in the number of cases of scarlet fever. The reduction in the number of cases of this disease, which had been noted in I 900 , ceased immediately the removal of patients to hospitals was stopped, although the total removed was but $6 \%$ lower than in the previous year. This happened during the annual cleaning of some of the wards.

"When to this is added the economy to householders if saved from a complete disinfection and renovation of houses, after two or three months' presence of scarlet fever, and the yet greater value to wage-earners of being able to go from their houses to continue their work, instead of loss of time through a forced quarantine, it will be apparent that nowhere, whether in a large city or in the country, can there be any question as to the advisability of having first cases of scarlet fever, like smallpox, removed to hospitals or a temporary building or tent, with, if necessary, the mother to act as nurse."

In the city of London, in $1891,18,381$ cases of scarlet fever were reported; of these 14,539 (78\%) were treated in hospitals, with a mortality of 542 $(3.73 \%)$. 


\section{CHAPTER XV}

\section{MEASLES}

BEFORE the time of Sydenham (1685) measles and scarlet fever were not, as a rule, recognised as distinct diseases and both of these eruptive fevers were commonly confounded with smallpox. Indeed it not unfrequently occurs even at the present day that cases of smallpox are diagnosed as measles at the outset of the attack; but the development of the characteristic eruption of smallpox soon makes it evident that a serious mistake has been made. The period of incubation in measles is from ten to twelve days, and the eruption usually appears on the third day after the initial symptoms are developed. These consist of fever and catarrhal symptoms, attended with cough and a watery discharge from the congested mucous membrane of the eyes and nose.

Measles, like scarlet fever, is a disease which has not been materially influenced by modern sanitary measures. The sanitary statistics of England and 
Wales show an increased mortality from this disease during the decade ending in 1890 , over the previous ten years (2.57 per 1000 deaths under five years of age in $1871-80$ and 3.13 in $1881-90$.) In the United States the total number of deaths from measles reported during the census year I900 was I2,866. The number of deaths in 1000 from all causes was 12.9 while in 1890 the proportion was II.I, showing a decided increase in this country also. More deaths occur in proportion to the population in cities (I8.2 per 100,000$)$ than in the country (9.9 per 100,000). The greatest mortality occurs among infants less than a year old ( 152.8 per 100,000 ) and among young children. After the age of five the mortality rate is greatly reduced (under five years 106.5 ; from five to fourteen years 7.4). The death-rate among the coloured population was somewhat greater than among native whites. "The greatest proportions of deaths from measles occurred in the South-west Central region ( $5 \mathrm{I} \cdot 7)$, the South Mississippi River belt (40.7), and the Southern Interior plateau (22.7); and the least in the Pacific Coast region (2.1), the Ohio River belt (6.3), and the Prairie region (6.5)" (Census Reports, 1900). The mortality from measles is to a large extent due to pulmonary complications, which are especially liable to occur in young children. The influence of external conditions in giving rise to these fatal complications 
(broncho-pneumonia, diphtheria) is shown by the fact that the greatest mortality occurs during the months of February, March, April, and May, and the least during the summer and autumn months, also by the very low mortality of the Pacific Coast region.

The combined influence of an unfavourable climate and density of population is shown by the statistics relating to the State of Rhode Island, which has the highest death-rate from this disease of any of the registration States (47.6). The lowest rate in the registration States was in Vermont (6. I).

Patients having measles may communicate the disease from the very outset of the attack and it is probable that the germ is present in the abundant secretion from the bronchial and nasal mucous membranes, as well as in the desquamated epithelium from the surface of the body after the eruption has developed. When convalesence has been established, if the patient's body and clothing have been disinfected he can no longer communicate the disease. The period during which isolation should be insisted upon, to prevent the extension of the disease, is about three weeks from the date of the first appearance of the eruption. The infectious material is not so tenacious of vitality as in smallpox and scarlet fever and the disease is not so likely to be conveyed by means of infected clothing and other articles exposed in the 
sick-room, if an interval of two or three weeks has elapsed since infection. This fact, however, should not lead to a neglect of the usual measures of disinfection heretofore recommended (see p. 220 and p. I 7 I). Free ventilation of the sick-room, after thorough scrubbing of surfaces with hot water and soap will, as a rule, ensure its disinfection. But it will be prudent not to allow susceptible children to enter such a room for at least fourteen days.

The following directions, published by the Board of Health of the city of Glasgow, set forth very forcibly the importance of preventing the spread of measles and the fact that isolation of the sick must be relied upon as the principal measure of prevention:

"Measles is a dangerous disease, one of the most dangerous with which a child under five years of age can be attacked. It is especially apt to be fatal to teething children. It tends to kill by producing inflammation of the lungs. It prepares the way for consumption. It tends to maim by producing inflammation of the eyes and ears.

"In Glasgow, during the last five years, measles has caused three deaths for every one which has been caused by scarlet fever; only one infectious disease has been more destructive to life, viz.: whooping-cough. Measles has carried off more than four times as many persons as enteric fever (typhoid).

"It is therefore a great mistake to look upon measles as a trifling disease.

"The older a child is the less likely is it to catch measles; and if it does, the less likely is it to die.

"If every child could be protected from measles until it had 
passed its fifth year, the mortality from measles would be enormously decreased.

"It is therefore a great mistake-because, as a rule, children sooner or later have measles-to say 'The sooner the better,' and to take no means to protect them, or even deliberately to expose them to infection.

"It is wrong for mothers with children in arms to go into houses where measles exists.

"Every child with measles ought at once to be put to bed and kept warm. The mildest cases may be made serious by a chill. Measles is for this reason most dangerous in winter and spring.

"A case of measles continues infectious for at least three weeks after the appearance of the rash. During that time separation from the healthy ought to be secured either by removal of the sick to hospital or by isolation at home.

"Isolation means not merely a separate room for the sick, but the withdrawal of apparently healthy children from school (day and Sunday) and the exclusion of strange children from the house.

"The isolation, as far as possible, from other children of all children belonging to the same family is more necessary in the case of measles than of any other infectious disease, because of this peculiarity-for days before the rash comes out, the child is highly infectious.

"School teachers, especially, ought to be familiar with the appearance of children in this stage of measles.

"The eyes are watery, glistening, and sensitive to light; there is a ringing cough, sneezing, and running from the nose, with flushed face; in short, all the signs of a bad cold in the head.

"No child showing these symptoms ought to be allowed to go to school.

"Any child observed at school with these symptoms ought to be sent home at once. Such children are to be looked for more particularly in the Infant Department.

"Sanitary Office,

“DR. J. B. RusSell.

"Montrose Street, Glasgow,

"January, $1897 . "$ 
Second attacks of measles are comparatively rare, even more so than second attacks of smallpox or of scarlet fever. The apparent exemption of adults from attacks of measles is largely if not altogether due to the fact that they have usually suffered an attack during childhood. It has been noticed that regiments of soldiers recruited in cities are less subject to measles than regiments raised in rural districts. This is no doubt due to the greater prevalence of the disease among children in cities, where few escape attack during infancy or the age of going to school.

During a severe epidemic in the Faroe Islands in I 846 scarcely any one escaped except those old enough to have passed through the previous epidemic in $178 \mathrm{I}$.

German measles (Rubella) is a distinct disease from measles, but its specific character was not generally recognised by physicians until the last half of the nineteenth century. An attack of this disease does not protect the child from the far more dangerous disease, measles.

The mortality from German measles is practically nil. Its prevention is therefore of much less importance, but is to be effected by the same measures, viz.: isolation of the sick and disinfection of all clothing and other objects which have been exposed in the sick-room. The period of incubation in this disease is quite variable, but as a rule it is longer than that of measles. 


\section{CHAPTER XVI}

\section{MALARIAL FEVERS}

THE discovery of the malarial parasite may justly 1 be considered one of the greatest achievements of scientific research during the nineteenth century. We owe it to Laveran, a surgeon in the French army, who made the discovery in 1880 while stationed in Algeria. His painstaking microscopical researches convinced him that the blood of patients suffering from malarial fever contains living amœboid parasites which in one stage of their development invade the red blood corpuscles and lead to their destruction. Subsequent researches in various parts of the world have made it evident that this blood parasite is in fact the malarial germ and the cause of the phenomena which characterise fevers of this class. It has also been demonstrated that the disease is transmitted to man by mosquitoes of the genus Anopheles, in the bodies of which the parasite passes through certain stages of development, 
resulting in the formation of a multitude of minute spore-like bodies which are found in the salivary glands of the insect.

Twenty-five years ago the best-informed physicians entertained erroneous views with reference to the nature of "malaria" and the cause of the malarial fevers. Observation had taught them that there is something in the air in the vicinity of marshes in tropical regions, and during the summer and autumn in semitropical and temperate regions, which gives rise to periodic fevers in those exposed in such localities; and the usual inference was that this something was of gaseous form - that it was a special kind of bad air (malaria) generated in swampy localities under favourable meteorological conditions. It was recognised at the same time that there are other kinds of bad air, such as the offensive emanations from sewers and the products of respiration of men and animals; but the term malaria was reserved for the kind of bad air which was supposed to give rise to the so-called malarial fevers. In the light of our present knowledge it is evident that the term is a misnomer. There is no good reason for believing that the air of swamps is any more deleterious to those who breathe it than the air of the sea-coast or that in the vicinity of inland lakes and ponds. Moreover, the stagnant ponds, which are 
covered with a "green scum" and from which bubbles of gas are given off, have lost all terrors for the well-informed man, except in so far as they serve as breeding-places for mosquitoes of the genus Anopheles. The green scum is made up of harmless algæ and the gas which is given off from the mud at the bottom of such stagnant pools is for the most part a well-known and comparatively harmless compound of hydrogen and carbon - methane or " marsh gas." In short, we now know that the air in the vicinity of marshes is not deleterious because of the presence of any special kind of bad air in such localities but because it contains mosquitoes infected with the malarial parasite.

The discoveries referred to, as is usual, have had to withstand the criticism of conservative physicians, who, having adopted the prevailing theories with reference to the etiology of periodic fevers, were naturally skeptical as to the reliability of the observations made by Laveran and those who claimed to have confirmed his discovery. The first contention was that the bodies described as present in the blood were not parasites, but deformed blood corpuscles. This objection was soon set at rest by the demonstration, repeatedly made, that the intra-corpuscular forms underwent distinct amœboid movements. No one witnessing these movements could doubt that he 
was observing a living micro-organism. The same was true of the extra-corpuscular flagellate bodies, which may be seen to undergo very active movements, as a result of which the red blood corpuscles are violently displaced and the flagellate body itself dashes about in the field of view.

The first confirmation in this country of Laveran's discovery of amœboid parasites in the blood of malarial-fever patients was made by myself in the path. ological laboratory of the Johns Hopkins University in March, I886. In May, I885, I had visited Rome as a delegate to the International Sanitary Conference, convened in that city under the auspices of the Italian Government, and while there I visited the Santo Spirito Hospital for the purpose of witnessing a demonstration, by Drs. Marchiafava and Celli, of that city, of the presence of the plasmodium malarice in the blood of persons suffering from intermittent fever. Blood was drawn from the finger during the febrile attack, from individuals to whom quinine had not been administered. The demonstration was entirely satisfactory, and no doubt was left in my mind that I saw living parasitic micro-organisms in the interior of red blood corpuscles obtained from the circulation of malarial-fever patients. The motions were quite slow, and were manifested by a gradual change of outline rather than by visible 
movement. After a period of amoboid activity of greater or less duration, the body again assumed an oval or spherical form and remained quiescent for a time. While in this form it was easily recognised, as the spherical shape caused the light passing through it to be refracted, and gave the impression of a body having a dark contour and a central vacuole, but when it was flattened out and undergoing amœboid changes in form it was necessary to focus very carefully and to have a good illumination in order to see it. The objective used was a Zeiss's one-twelfth inch homogeneous oil immersion.

Very properly, skepticism with reference to the causal relation of these bodies to the disease with which they are associated was not removed by the demonstration that they are in fact blood parasites, that they are present in considerable numbers during the febrile paroxysms, and that they disappear during the interval between these paroxysms. These facts, however, give strong support to the inference that they are indeed the cause of the disease. This inference is further supported by the evident destruction of red blood corpuscles by the parasite, as shown by the presence of grains of black pigment in the amœba-like micro-organisms observed in these corpuscles and the accumulation of this insoluble blood pigment in the liver and spleen of those who 
have suffered repeated attacks of intermittent fever. The enormous loss of red blood corpuscles as a result of such attacks is shown by the anæmic condition of the patient and also by actual enumeration. According to Kelsch, a patient of vigorous constitution in the first four days of a quotidian intermittent fever, or a remittent of first invasion, may suffer a loss of 2,000,000 of red blood corpuscles per cubic millimetre of blood, and in certain cases a loss of I, $, 00,000$ has been verified at the end of twenty-four hours. In cases of intermittent fever having a duration of twenty to thirty days the number of red blood cells may be reduced from the normal, which is about $5,000,000$ per cubic millimetre, to $1,000,000$ or even less.

In view of this destruction of the red blood cells and the demonstrated fact that a certain number at least are destroyed during the febrile paroxysms by a blood parasite which invades the cells and grows at the expense of the contained hæmoglobin, it may be thought that the causal relation of the parasite should be conceded. But scientific conservatism demands more than this and the final proof has been afforded by the experiments of Gerhardt and of Marchiafava and Celli - since confirmed by many others. This proof consists in the experimental inoculation of healthy individuals 
with blood containing the parasite and the development of a typical attack of periodic fever as a result of such inoculation. After such an inoculation a period varying from four to twenty-one days elapses before the occurrence of a febrile paroxysm. This is the so-called period of incubation, during which, no doubt, the parasite is undergoing multiplication in the blood of the inoculated individual. The duration of this period depends to some extent upon the quantity of blood used for the inoculation and its richness in parasites. It also depends upon the particular variety of the parasite present, for it has been ascertained that there are at least three distinct varieties of the malarial parasite - one which produces the quartan type of fever, in which there is a paroxysm every third day and in which, in experimental inoculations made, the period of incubation has varied from eleven to eighteen days; one in which the paroxysm occurs every second day (tertian), in which the period of incubation is from nine to twelve days; and one, denominated the xstivo-autumnal type, in which the period of incubation rarely exceeds five days.

The parasite associated with each of these types may be recognised by an expert, and there is no longer any doubt that the difference in type is due to the fact that different varieties or "species" 
of the malarial parasite exist, each having a different period of development. Blood drawn during a febrile paroxysm shows the parasite in its different stages of intra-corpuscular development. The final result of this development is a segmenting body, having pigment granules at its centre, which occupies the greater part of the interior of the red blood corpuscle. The number of segments into which this body divides differs in the different types of fever, and there are other points of difference by which the several varieties may be distinguished one from the other, but which it is not necessary to mention at the present time. The important point is that the result of the segmentation of the adult parasites contained in the red corpuscles is the formation of a large number of spore-like bodies, which are set free by the disintegration of the remains of the blood corpuscles and which constitute a new brood of reproductive elements, which in their turn invade healthy blood corpuscles and effect their destruction. This cycle of development, without doubt, accounts for the periodicity of the characteristic febrile paroxysms; and, as stated, the different varieties complete their cycle of development in different periods of time, thus accounting for the recurrence of the paroxysms at intervals of forty-eight hours in one type of fever and of three 
days in another type. When a daily paroxysm occurs, this is believed to be due to the alternate development of two groups of parasites of the tertian variety, as it has not been possible to distinguish the parasite found in the blood of persons suffering from a quotidian form of intermittent fever from that of the tertian form. Very often, also, the daily paroxysm occurs on succeeding days at a different hour, while the paroxysm every alternate day is at the same hour, a fact which sustains the view that we have to deal, in such cases, with two broods of the tertian parasite which mature on alternate days. In other cases there may be two distinct paroxysms on the same day and none on the following day, indicating the presence of two broods of tertian parasites maturing at different hours every second day.

The hypothesis that malarial infection results from the bites of mosquitoes was advanced and ably supported by Dr. A. F. A. King, of Washington, D. C., in a paper read before the Philosophical Society on February 10, 1883, and published in the Popular Science Monthly in September of the same year. In I894 Manson supported the same hypothesis in a paper published in the British Medical Journal (December 8th), and the following year (1895) Ross made the important discovery that when blood containing the crescentic bodies was ingested by the mosquito 
these crescents rapidly underwent changes resulting in the formation of motile filaments, which become detached from the parent body and continue to exhibit active movements. In 1897 Ross ascertained further that when blood containing crescents was fed to a particular species of mosquito, living pigmented parasites could be found in the stomach walls of the insect. Continuing his researches with a parasite of the same class which is found in birds, and in which the mosquito also serves as an intermediate host, Ross found that this parasite enters the stomach wall of the insect, and, as a result of its development in that locality, forms reproductive bodies (sporozoites), which subsequently find their way to the veneno-salivary glands of the insect, which is now capable of infecting other birds of the same species as that from which the blood was obtained in the first instance. Ross further showed that the mosquito which served as an intermediate host for this parasite could not transmit the malarial parasite of man or another similar parasite of birds (halteridium). These discoveries of Ross have been confirmed by Grassi, Koch, and others, and it has been shown that the mosquitoes which serve as intermediate hosts for the malarial parasites of man belong to the genus Anopheles, and especially to the species known as Anopheles claviger. 
The question whether malarial fevers can be contracted in any other way than through inoculation by infected mosquitoes, or direct experimental inoculation with the blood of one suffering from malarial infection, has been submitted to experimental investigation, and the answer is in the negative. Formerly it was believed by many physicians that malarial fevers might be contracted by drinking surface water obtained in malarious localities, but there is no experimental evidence in favour of this hypothesis. The first experiment of the nature indicated was made in the summer of 1900 , and the results were reported by Manson in September of that year.

Five healthy individuals lived in a hut on the Roman Campagna since early in the month of July. They were protected against mosquito bites by mosquito-netting screens in the doors and windows and by mosquito-bars over the beds. They went about freely during the daytime, but remained in their protected hut from sunset to sunrise. At the time Manson made his report all these individuals remained in perfect health. It has long been known that labourers could come from the villages in the mountainous regions near the Roman Campagna and work during the day, returning to their homes at night, without great danger of contracting the fever, while those who remained on the Campagna at night ran great risk of 
falling sick with fever, as a result of "exposure to the night air." What has already been said makes it appear extremely probable that the "night air," per se, is no more dangerous than the day air, but that the real danger consists in the presence of infected mosquitoes of a species which seeks its food at night. As pointed out by King, in his paper already referred to, it has repeatedly been claimed by travellers in malarious regions that sleeping under a mosquito-bar is an effectual method of prophylaxis against intermittent fevers.

An experiment on a larger scale has since been made by some medical officers of the Japanese army on the island of Formosa, where two companies of soldiers were stationed in a very malarious locality. The men of one company were carefully protected from the bites of mosquitoes and did not suffer attacks of malarial fever, while those of the other company, who were not protected from mosquito bites, suffered severely.

The geographic distribution of the malarial fevers is coextensive with the conditions favourable for the development of the mosquito which serves as an intermediate host of the parasites to which the disease is due. Accordingly we find that these fevers prevail in tropical regions, where there is abundant moisture, in all parts of the world; and in temperate regions, 
during the summer months, wherever there are suitable breeding-places for mosquitoes of the genus Anopheles.

The more severe and fatal forms of malarial infection are found especially in low-lying regions in the tropics. In the United States malarial fevers are common along the Gulf and South Atlantic coasts, and in the valleys of rivers throughout the Southern and South-western States. There is considerable malaria along the shores of lakes Ontario and Erie, but fevers of this class are rare in the vicinity of lakes Superior and Michigan. The disease prevails in a mild form in some portions of the States of New York and Pennsylvania but is extremely rare in New England and is almost unknown on the Pacific Coast. Many localities in the United States which furnished numerous cases of malarial fever when first settled have since become comparatively healthy as a result of agricultural operations by which marshy lands have been drained and reclaimed.

The seasonal prevalence differs in different regions, but in the temperate zone is usually greatest during the summer and early autumn. A considerable rainfall during the spring and summer months leads to the formation of stagnant pools and marshes which serve as breeding-places for mosquitoes, but an excessive rain-fall, by which swamps and ponds are kept 
full of water, is not favourable for the multiplication of mosquitoes and it has long been known that malarial fevers are less prevalent under such circumstances. The total number of deaths from malarial fever during the census year 1900 was 14,874 and the proportion per 1000 deaths from all causes was 14.9. The proportion during the census year 1890 was considerably greater(22.1). The popular idea that the negro is less susceptible to malarial fever than the white man does not appear to receive support from the census returns as the mortality among the coloured was 59.8 per 100,000 of population and among whites 6.5 per 100,000 . But it must be remembered that the coloured population of the United States is located to a much greater extent than the whites in the more malarious regions of the country. Another factor which probably vitiates the statistics to a considerable extent is the fact that many deaths ascribed to malarial fever are doubtless due to typhoid fever. This mistake in diagnosis has frequently been made in all parts of the world and is especially liable to occur among an ignorant population. The mortality from malarial fevers does not, however, fairly represent the mischief accomplished by malaria-infected mosquitoes.

The forms of malarial fever commonly encountered in the United States are rarely fatal, especially if 
proper treatment is resorted to (the administration of quinine). But the infection is very persistent, relapses are frequent, and in malarious regions many individuals often suffer for years from chronic malarial infection and finally succumb to some chronic organic disease resulting from repeated attacks of fever, or from some acute disease for which a predisposition has been established as a result of continued ill-health and the anæmia which is characteristic of chronic malarial poisoning. The greatest proportional number of deaths from malarial fever, within the limits of the United States, during the census year I900 occurred in the "South Mississippi River belt" ( 88.8 per 1000 deaths from all causes); the next greatest in the "South Atlantic Coast region" (61.7); the next greatest in the "South-west Central region" (57.9); next in the "Gulf Coast region" (47.9); next in the "Southern Interior plateau" (43.8). The least mortality occurred in the region of the "Great Northern Lakes" (2.2); next in the "North Atlantic Coast region" (2.3); next in the "Central Appalachian region" (2.6); next in the "Northeastern hills and plateaus" (2.8); next in the "Pacific Coast region" (2.9).

Now that we know the life history of the malarial parasite and the manner by which it gains access to the human body, the proper preventive measures are 
apparent. In general these consist in destroying the breeding-places of mosquitoes when practicable, in destroying the ova and larvæ in marshes and pools of stagnant water, and in avoiding the bites of the insects.

In bodies of water containing minnows and other small fish the larva of mosquitoes are promptly distroyed, as they serve them as food. Frogs also feed upon mosquito larvæ. Mosquitoes do not, as a rule, deposit their eggs in running streams and a perfectly smooth surface of water is required for the exit of the adult mosquito from the puparium. It is said that this may be effectually prevented by agitating the surface of the water with a water-wheel put in motion by a wind-mill. There are various temporary expedients by which the larvæ may be killed in the breeding-places of mosquitoes, but evidently the most effectual remedy is to destroy the breeding-places by filling up stagnant pools, filling or draining marshes, and removing all small receptacles of water from the vicinity of dwellings. Rain-water barrels, bottles, tin cans, street catch-basins, etc., all serve as breeding places, especially for mosquitoes of the genus Anopheles which serve as the intermediate host of the malarial parasite. As a temporary measure the use of petroleum is to be especially recommended. This quickly spreads out over the surface of bodies of 
stagnant water, whether large or small, and destroys the larvæ, which are obliged to come to the surface from time to time for air. An ounce of oil is said to be sufficient for fifteen square feet of surface, and an application of this amount to be effective for about two weeks.

The destruction of adult mosquitoes near dwellings may be to some extent accomplished by placing lighted lamps, in plates containing petroleum, at a little distance from the house. The mosquitoes fly about the lamps and many of them are likely to fall into the petroleum, which quickly kills them. The task of killing mosquitoes inside of a house is, however, far easier than that of destroying those on the outside. Their entrance should as far as possible be prevented by the use of window and door screens. Those which pass these barriers should be destroyed by burning, in the closed apartments, pyrethrum powder, or by fumigation with formaldehyd gas or sulphur dioxid (formed by burning sulphur). It is especially important that all mosquitoes should be killed in the autumn in houses located in malarious regions. Otherwise they are likely to hibernate and the females will serve in the spring to start a new generation of annoying and possibly dangerous pests. They are to be found not only in bedrooms, but in cellars, kitchens, closets, and attics. 
For the protection of individuals from the bites of mosquitoes, infected or otherwise, a mosquito-bar at night and a suitable veil of mosquito-netting in the daytime will be the most reliable resource. Various substances which are obnoxious to the insects may also be rubbed upon exposed portions of the body. Of these oil of eucalyptus is perhaps the most efficacious. The following prescription is said to serve a useful purpose when applied to the hands and face: Ether and alcohol, of each five parts; cologne water and oil of eucalyptus, of each ten parts; tincture of pyrethrum fifteen parts. This is to be diluted, before it is used, with four or five parts of water to one of the mixture. An infusion of quassia is also said to be useful for the same purpose.

If by the measures heretofore referred to the bites of infected mosquitoes can be avoided no other prophylaxis will be required. But those who are unavoidably exposed in malarious regions and who, with all possible precautions, are unable to escape the bites of these insects will do well to take quinine in doses of six to ten grains during the day at intervals of four or five days. This is believed to be better than a daily dose of a smaller amount. 


\section{CHAPTER XVII}

\section{YELLOW FEVER}

YELLOW fever is an infectious disease which has a comparatively restricted geographic range. Occasional epidemics have occurred in North America in every one of our seaport cities as far north as Boston and on the Mississippi River as far north as St. Louis, but it has never established itself as an endemic disease within the limits of the United States. The cities of Havana, Vera Cruz, and Rio de Janeiro have been the principal foci of the disease for many years, and from these cities it has been carried to the seaports of North and South America and of the West Indies. It has also prevailed for many years on the west coast of Africa, which by some authorities is regarded as the original source of this pestilential malady, while others believe that it already existed in the West Indies at the time of the discovery of these islands. The disease has never reached the east coast of Africa, and is unknown in 
Asia. In Europe its ravages have been restricted to Spain, to which country it has several times been introduced by means of ships coming from the West Indies.

In the United States several severe epidemics occurred in the city of Philadelphia during the latter part of the eighteenth century (I 793, I 797, I 798), but since that time the ravages of the disease have, for the most part, been confined to more southern localities.

During the first sixty years of the past century it prevailed almost annually in one or more of the Southern seaports of the United States and not infrequently it extended its ravages to the interior towns in one or more of the Southern States. So frequently did it prevail during the summer months in New Orleans and Charleston that the permanent residents of those cities commonly regarded it as a disease of the climate and a necessary evil which it was folly to attempt to combat by quarantine restrictions.

In the great epidemic of 1853 , yellow fever prevailed extensively in the States of Florida, Alabama, Louisiana, Mississippi, Arkansas, and Texas. The epidemic of 1867 was limited to the States of Louisiana and Texas. Those States again suffered severely in 1873 , and the States of Florida, Alabama, and 
Mississippi were also invaded. A still more extended and deadly epidemic occurred in 1878 , causing a mortality of 15,934 out of a total number of cases exceeding 74,000 . In this epidemic the disease followed the Mississippi River to the very suburbs of St. Louis, and the State of Tennessee suffered severely as well as the States south of it. The city of Memphis alone had a mortality from the disease of about five thousand. These repeated epidemics not only cost the lives of thousands of citizens and paralysed business of all kinds during their prevalence, but apprehension with reference to the recurrence of the disease very materially interfered with the growth of many Southern cities and retarded greatly the development of those portions of the country most liable to invasion. All this is now changed; public health officials are no longer filled with apprehension upon the approach of summer by the thought that any ship arriving from Havana may introduce the deadly pestilence to our shores; commerce is no longer subjected to the serious restrictions formerly considered necessary for the exclusion of the disease; and the public generally have been made aware that the fangs of this threatening monster have been drawn by the scientific demonstration of its mode of attack and the simple measures which have been proved to be effective in preventing its propagation. 
The demonstration that yellow fever is propagated from man to man by mosquitoes of the genus Stegomyia was made by a board of medical officers of the United itates army, which was appointed upon the recommendation of the present writer, and which prosecuted its researches in Cuba during the years I 900 and I90I. This board consisted of Major Walter Reed, Surgeon U. S. A. ; Dr. James Carroll, Contract Surgeon U. S. A. ; Dr. A. Agramonte, Contract Surgeon U.S. A. ; and Dr. Jesse W. Lazear, Contract Surgeon U. S. A.

In a "Preliminary Note," read at the meeting of the American Public Health Association, October 22, 1900, the board gave a report of three cases of yellow fever which they believed to be the direct result of mosquito inoculations. Two of these were members of the board, viz., Dr. Jesse W. Lazear and Dr. James Carroll, who voluntarily submitted themselves to the experiment. Dr. Carroll suffered a severe attack of the disease and recovered, but Dr. Lazear fell a victim to his enthusiasm in the cause of science and humanity. His death occurred on September $25^{\text {th }}$, after an illness of six days' duration. About the same time nine other individuals who volunteered for the experiment were bitten by infected mosquitoes-i.e., by mosquitoes which had previously been allowed to fill themselves with blood from yellow 
fever patients - and in these cases the result was negative. In considering the experimental evidence thus far obtained, the attention of the members of the board was attracted by the fact that in the nine inoculations with a negative result, "the time elapsing between the biting of the mosquito and the inoculation of the healthy subject varied in seven cases from two to eight days and in the remaining two from ten to thirteen days, whereas in two of the three successful cases the mosquito had been kept for twelve days or longer."

The inference drawn by Dr. Reed and his associates, from the experiments thus far made, was that yellow fever may be transmitted by mosquitoes of the genus Stegomyia, but that in order to convey the infection to a non-immune individual the insect must be kept for twelve days or longer after it has filled itself with blood from a yellow-fever patient in the earlier stages of the disease. In other words, that a certain period of incubation is required in the body of the insect before the germ reaches its salivary glands, and consequently before it is able to inoculate an individual with the germs of yellow fever. This inference, based upon experimental data, received support from other observations, which have been repeatedly made, with reference to the introduction and spread of yellow fever in localities favourable to its propagation. 
When a case is imported into one of our Southern seaport cities from Havana, Vera Cruz, or some other endemic focus of the disease, an interval of two weeks or more occurs before secondary cases are developed as a result of such importation. In the light of our present knowledge this is readily understood. A certain number of mosquitoes having filled themselves with blood from this first case, after an interval of twelve days or more, bite non-immune individuals living in the vicinity, and these individuals, after a brief period of incubation, fall sick with the disease; being bitten by other mosquitoes they serve to transmit the disease through the "intermediate host" to still others. Thus the epidemic extends, at first slowly from house to house, then more rapidly, as by geometrical progression. The results reported by Dr. Reed and his associates have since been fully confirmed by their subsequent experiments and by independent investigations made in Cuba and also in Brazil.

Before the discovery that yellow fever is transmitted by mosquitoes, this disease was generally regarded as one of the filth diseases, although there were many facts opposed to this view. In the light of our present knowledge we can no longer class it with typhoid fever, cholera, bubonic plague, and dysentery, in which diseases the germ is known to be 
present in the alvine discharges of the sick, and which are, consequently, well named filth diseases.

We now see clearly, however, why in certain particulars relating to its etiology it resembles the malarial fevers. It is limited as regards its prevalence to comparatively warm latitudes or to the summer months in more temperate regions and is dependent, to a certain extent, upon rainfall or the proximity of standing water, because these conditions are necessary for the propagation of mosquitoes.

It is evident that in view of our present knowledge relating to the mode of transmission of yellow fever, the preventive measures which have heretofore been considered most important-i.e., isolation of the sick, disinfection of clothing and bedding, and municipal sanitation-are either of no avail or of comparatively little value. It is true that yellow-fever epidemics have resulted, as a rule, from the introduction to a previously healthy locality of one or more persons suffering from the disease. But we now know that its extension did not depend upon the direct contact of the sick with non-immune individuals and that isolation of the sick from such contact is unnecessary and without avail. On the other hand, complete isolation from the agent which is responsible for the propagation of the disease is all-important. In the absence of a yellow-fever patient from which to draw 
blood the mosquito is harmless, and in the absence of the mosquito the yellow-fever patient is harmless-as the experimental evidence now stands. Yellow-fever epidemics are terminated by cold weather, because then the mosquitoes die or become torpid. The sanitary condition of our Southern seaport cities is no better in winter than in summer, and if the infection attached to clothing and bedding it is difficult to understand why the first frosts of autumn should arrest the progress of an epidemic. But all this is explained now that the mode of transmission has been demonstrated.

Insanitary local conditions may, however, have a certain influence in the propagation of the disease, for it has been ascertained that the species of mosquito which serves as an intermediate host for the yellow-fever germ may breed in cesspoobls and sewers, as well as in stagnant pools of water. If, therefore, the streets of a city are unpaved and ungraded and there are open spaces where water may accumulate in pools, as well as open cesspools to serve as breeding-places for Stegomyia fasciatus, that city will present conditions more favourable for the propagation of yellow fever than it would if well paved and drained and sewered.

It will be remembered that the malarial fevers are contracted as a result of inoculation by mosquitoes 
of the genus Anopheles, and that the malarial parasite has been demonstrated not only in the blood of those suffering from malarial infection, but also in the stomach and salivary glands of the mosquito. If the yellow-fever parasite resembled that of the malarial fevers it would no doubt have been discovered long ago. But as a matter of fact, this parasite, which we now know is present in the blood of those sick with the disease, has thus far eluded all researches. Possibly it is ultra-microscopic.

Individuals of every race and of all ages, who are exposed to infection for the first time, during the epidemic prevalence of the disease, are subject to be attacked. But there is a wide difference in the degree of this susceptibility among races, and among individuals of the same race.

It has been asserted that the negro race has a congenital immunity from yellow fever, but this is a mistake. The susceptibility of the negro is, however, much less than that of the white race, and among those attacked the mortality, as a rule, is small.

Immunity is acquired by suffering an attack of the disease; this acquired immunity is not, however, absolute.

Second attacks no doubt occasionally occur, although this has been denied by some authors.

The proper measures of prophylaxis are given in 
the following circular, which was submitted for my approval by the chief surgeon, Department of Cuba, and was published by the commanding general of that department.
$\left.\begin{array}{c}\text { "Circular } \\ \text { "No. 5. }\end{array}\right\}$
"Headquarters Department of Cuba, "Havana, April 27, Igor.

"Upon the recommendation of the chief surgeon of the department, the following instructions are published and will be strictly enforced at all military posts in this department:

"The recent experiments made in Havana by the Medical Department of the Army having proved that yellow fever, like malarial fever, is conveyed chiefly, and probably exclusively, by the bite of infected mosquitoes, important changes in the measures used for the prevention and treatment of this disease have become necessary.

"I. In order to prevent the breeding of mosquitoes and to protect officers and men against their bites, the provisions of General Orders No. 6, Department of Cuba, December 21, 1900, shall be carefully carried out, especially during the summer and fall.

" 2. So far as yellow fever is concerned, infection of a room or building simply means that it contains infected mosquitoes, that is, mosquitoes which have fed on yellow-fever patients. Disinfection, therefore, means the employment of measures aimed at the destruction of these mosquitoes. The most effective of these measures is fumigation, with either sulphur, formaldehyd, or insect powder. The fumes of sulphur are the quickest and most effective insecticide but are otherwise objectionable. Formaldehyd gas is quite effective if the infected rooms are kept closed and sealed for two or three hours. The smoke of insect powder has also been proved very useful; it readily stupefies mosquitoes, which drop to the floor and can then be easily destroyed.

"The washing of walls, floors, ceilings, and furniture with disinfectants is unnecessary.

"3. As it has been demonstrated that yellow fever cannot be 
conveyed by fomites, such as bedding, clothing, effects, and baggage, they need not be subjected to any special disinfection. Care should be taken, however, not to remove them from the infected rooms until after formaldehyd fumigation, so that they may not harbour infected mosquitoes.

"Medical officers taking care of yellow-fever patients need not be isolated; they can attend other patients and associate with non-immunes with perfect safety to the garrison. Nurses and attendants taking care of yellow-fever patients shall remain isolated, so as to avoid any possible danger of their conveying mosquitoes from patients to non-immunes.

"4. The infection of mosquitoes is most likely to occur during the first two or three days of the disease. Ambulant cases, that is, patients not ill enough to take to their beds and remaining unsuspected and unprotected, are probably those most responsible for the spread of the disease. It is therefore essential that all fever cases should be at once isolated and so protected that no mosquitoes can possibly get access to them until the nature of the fever is positively determined.

"Each post shall have a 'reception ward' for the admission of all fever cases and an 'isolation ward' for the treatment of cases which prove to be yellow fever. Each ward shall be made mosquito-proof by wire netting over doors and windows, a ceiling of wire netting at a height of seven feet above the floor, and mosquito-bars over the beds. There should be no place in it where mosquitoes can seek refuge, not readily accessible to the nurse. Both wards can be in the same building, provided they are separated by a mosquito-tight partition.

" 5. All persons coming from an infected locality to a post shall be kept under careful observation until the completion of five days from the time of possible infection, either in a special detention camp or in their own quarters; in either case their temperature should be taken twice a day during this period of observation, so that those who develop yellow fever may be placed under treatment at the very inception of the disease.

"6. Malarial fever, like yellow fever, is communicated by mosquito bites and therefore is just as much of an infectious 
disease and requires the same measures of protection against mosquitoes. On the assumption that mosquitoes remain in the vicinity of their breeding places, or never travel far, the prevalence of malarial fever at a post would indicate want of proper care and diligence on the part of the surgeon and commanding officer in complying with General Orders No. 6, Department of Cuba, 1900.

"7. Surgeons are again reminded of the absolute necessity, in all fever cases, to keep, from the very beginning, a complete chart of pulse and temperature, since such a chart is their best guide to a correct diagnosis and the proper treatment.

"By Command of Major-General Wood.

"H. L. SCOTT, "Adjutant-General."

The practical execution, in the city of Havana, by Major ${ }^{1}$ Gorgas, Surgeon U. S. A., chief sanitary officer of the city during its occupation by our troops, of the measures indicated in the above circular was attended with entire success. For more than two years this city has been entirely free from cases of yellow fever, while for many years prior to the date when Major Gorgas inaugurated his war upon infected mosquitoes the disease had prevailed to a greater or less extent annually and a certain number of deaths had occurred every month in the year.

${ }^{1}$ Now Colonel Gorgas, by special act of Congress, as a reward for his services. 


\section{CHAPTER XVIII}

WOUND INFECTIONS

CERTAIN of the diseases heretofore considered may be communicated to man by inoculation and are occasionally contracted by the accidental inoculation of wounds or abrasions. Thus we may have a tubercular infection of the skin ("lupus") extending sometimes to adjacent lymphatic glands, or a localised diphtheritic process, upon any portion of the surface of the body. But in the present chapter we propose to consider certain localised or general infectious diseases which as a rule have their origin through the accidental introduction of pathogenic bacteria into an open wound or upon an abraded surface. Before the days of antiseptic surgery such accidental inoculations were much more frequent than at present. Erysipelas, hospital gangrene, suppuration, septicæmia ("blood-poisoning"), and tetanus were of frequent occurrence and the mortality from certain surgical operations which are now almost free 
from risk was often excessive. These facts are well known to the public and it is also generally known that when a wound made by the surgeon, or the result of accident, suppurates or gives rise to fever, it is because it has become infected. The germs which usually give rise to wound infection have been carefully studied by bacteriologists and are now well known. The two most common species, which are responsible to a large extent for the suppuration of wounds, for erysipelatous inflammation, and for "bloodpoisoning," are widely distributed and are commonly found upon the surface of the body and of mucous membranes in healthy persons. One of these is a micrococcus which, when cultivated in artificial media, is recognised by the fact that it forms masses of a golden-yellow colour. These masses are made up of minute spherical cells which adhere to each other in irregular grape-like bunches-hence the technical name Staphylococcus pyogenes aureus. The other is also a micrococcus in which the spherical cells are united in chains, like strings of pearls. This is called Streptococcus pyogenes. This latter is also called the streptococcus of erysipelas because it has been demonstrated to be the cause of erysipelatous inflammations. These two species of pathogenic bacteria give rise to a great variety of infectious processes. Both are found, either separately or associated, in the pus of 
abscesses, in boils and carbuncles, in suppurating wounds, in puerperal fever, in peritonitis, in suppurative disease of the ear, in general blood-poisoning, etc.

As in other infectious diseases infection by these pathogenic micrococci (so-called " pus cocci") depends upon three factors, viz. : the virulence of the germ;

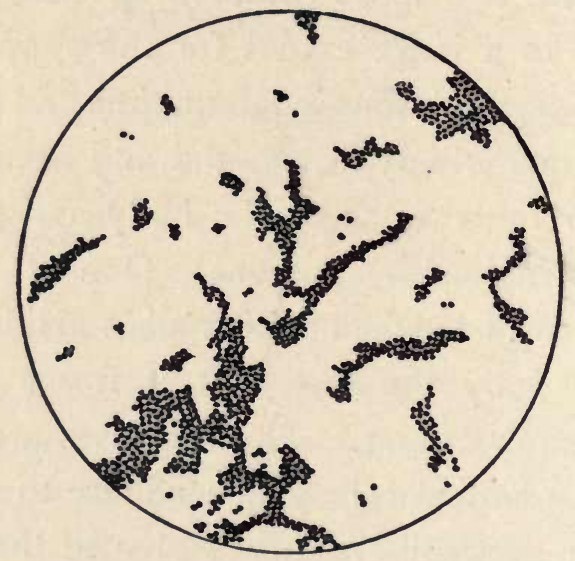

FIG. I0. Micrococcus of pus-formation (Staphylococrus pyogenes aureus); magnified 1000 diameters.

the vital resistance of the tissues invaded; and the number of germs introduced into an open wound. The virulence of the germs is much greater when they come from a suppurating wound, from a case of erysipelas or of puerperal fever, or, in short, from any infectious process in the body, than when they come from the mouth or the surface of the body where they 
have been living a saprophytic existence. Here it may be necessary to explain that a saprophytic bacterium is one which exists independently of a living host and which obtains its supply of nutriment from dead animal or vegetable material, while a parasitic bacterium is one which invades the body of a living animal and receives its nourishment at the expense of

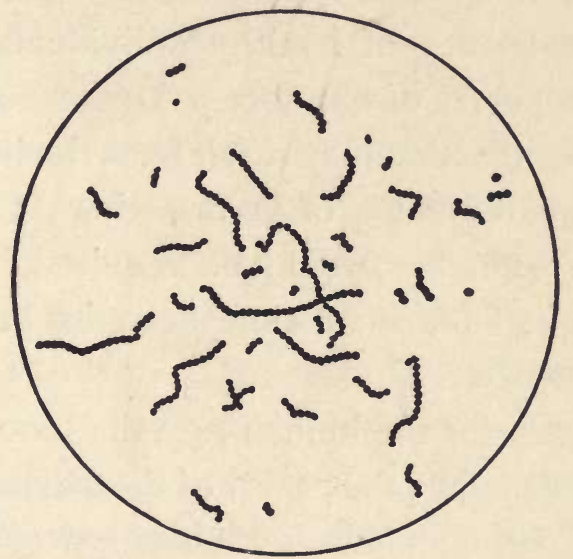

FIG. II. Micrococcus of erysipelas, etc. (Streptococcus pyogenes); magnified I000 diameters.

the tissues and body fluids of its "host." But certain bacteria, like those at present under consideration, may live either as saprophytes or as parasites.

Their ability to effect a lodgment in the tissues and multiply there, after having led a saprophytic life for some time, is favoured by a reduction in the vital resisting power of the individual as a result of various 
depressing agencies - alcoholism, insufficient nourishment, loss of blood, etc.; or by a diminished vital resistance at the point of invasion as a result of injury to the tissues by bruising, by burns, by various chemical agents, etc.

When, however, these bacteria have been leading a parasitic life for some time they have a greatly increased virulence, as manifested by their ability to invade the tissues of healthy individuals whenever they find a portal of entrance. Under such circumstances, also, infection may result from the introduction of a very small number of germs, whereas a comparatively large number would be required in case the micrococci had for some time been leading a saprophytic existence.

Fortunately for the human race the blood serum of healthy persons has the power of destroying a limited number of pathogenic bacteria of a low grade of virulence. But when these germs come directly from the seat of an infectious process, especially in the case of the streptococcus, a very small number may give rise to a rapidly extending and deadly blood infection for example the blood-poisoning resulting from puncture of the skin with a needle during an autopsy of a case of erysipelas or of puerperal fever, or from a surgical operation upon a patient suffering from any form of streptococcus infection. Many pathologists 
and surgeons have suffered serious and often fatal results from such an apparently insignificant wound.

Antiseptic surgery has for its object the destruction of all bacteria in wounds or attached to objects which are brought into contact with wounds, such as the hands and instruments of the surgeon, surgical dressings, etc. This is accomplished by the use of chemical agents of established germicidal value, which must be used in such a proportion as will insure the destruction of germs, and which will not have an injurious effect upon the vitality of the tissues and the healing process. The principal agents which have been used for this purpose are carbolic acid and corrosive sublimate (mercuric chloride) in solutions of proper strength. There are certain objections to the use of either of these agents in solutions strong enough to promptly destroy disease germs, especially when applied to wounds of considerable magnitude. For this reason antiseptic surgery has to a considerable extent been superseded by aseptic surgery, which accomplishes the same result without the application of chemical agents of any kind to the wound surfaces. Instruments and dressings are rendered sterile by heat, usually in a steam steriliser. The hands of the surgeon and the "field of operation" are thoroughly scrubbed with soap and water and then washed in an antiseptic solution to insure the destruction of germs 
attached to the skin. Usually this is followed by washing with alcohol and sterile water to remove all traces of the antiseptic. Many surgeons at the present day prefer to wear india-rubber gloves while operating, as it has been found by experience that it is a difficult matter to thoroughly sterilise the hands. Such gloves are easily cleaned and sterilised. By the "field of operation" is meant the surface of the body in the vicinity of the incisions which are to be made in any surgical operation. This surface is cleaned as thoroughly as possible and other portions of the body are covered with a clean sheet or sterile towels. When the operating-room and its fixtures are "surgically clean" and all necessary precautions are taken with reference to instruments, dressings, the surgeon's hands, etc., an operation wound is expected to heal promptly by adhesion of the wound surfaces, which have been brought together and retained by sutures or adhesive plaster, and bandages when necessary.

Such wounds treated aseptically rarely suppurate. When they do it is because some of the bacteria which cause pus formation have found their way into the wound in spite of the precautions taken. It is more difficult to prevent suppuration in gunshot wounds and in extensive lacerations resulting from railway accidents, etc. The bruising of the tissues in 
such cases renders them less able to resist infection and less apt to unite by adhesion.

It often happens, also, that the wound is infected at the time it is inflicted. Thus in a gunshot wound a portion of the clothing to which numerous germs are attached may be carried into the wound. Or the bullet itself may be infected, although this is no doubt of rare occurrence.

Lacerated wounds are often inflicted with stones, pieces of wood, or other objects which have dirt adhering to them, which may contain various pathogenic bacteria, and which are liable to remain lodged in the wound. Such a wound may be cleansed and rendered aseptic by being thoroughly washed with sterile water (boiled or distilled), or it may be washed with an antiseptic solution and treated with antiseptic dressings to prevent the development of any bacteria which may chance to remain hidden away in the wound. 


\section{CHAPTER XIX}

\section{TETANUS}

\section{A FORM of wound infection of special interest, be- cause of the very serious results which usually}

follow such infection, is that by the bacillus of tetanus, which gives rise to the disease commonly known as lockjaw.

The bacillus of tetanus was discovered in 1884 , by a student (Nicholaer) in the laboratory of Professor Flügge of Göttingen. Having introduced small quantities of garden earth under the skin of mice and of guinea-pigs, some of these animals died with all the characteristic symptoms of tetanus. Subsequent researches have established the fact that in temperate and tropical regions the bacillus of tetanus is widely distributed and is commonly present in rich soil which has been manured. It is also present in the dust of city streets, and there is good reason to believe that its being found there is due to the fact that it is present in the intestinal contents of horses. 
Formerly tetanus was supposed to be due to injury to the nerves, and the idea that it is an infectious disease, due to the introduction of a specific bacillus into a wound, had not been entertained prior to the demonstration that the symptoms which characterise this disease may be produced in the lower animals by inoculating them with garden earth or with a pure

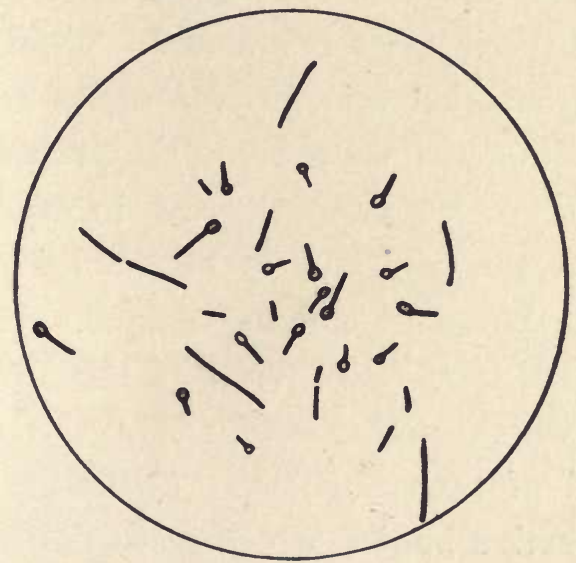

FIG. 12. Bacillus of tetanus; magnified rooo diameters. Spores are seen at the ends of some of the bacilli.

culture of the tetanus bacillus. The nervous symptoms are now known to be due to the fact that the tetanus bacillus produces a deadly poison (toxin) which has a special affinity for the nervous tissues. When a barefooted boy steps upon a rusty nail and, as a result of the penetrating wound inflicted by it, develops tetanus, this result is not due to the fact that the nail 
was rusty, or that a nerve had been injured, but to the introduction of earth containing the tetanus bacillus, which would more readily adhere to the rough surface of a rusty nail than to a new and clean one. The nature of the wound made is also favourable to infection, as the bruised tissues are not likely to bleed much, and the deep wound with a narrow orifice is well calculated to retain any foreign matter introduced at the time the injury was inflicted. Unlike the various pathogenic bacteria heretofore referred to, the tetanus bacillus will not grow in the presence of oxygen. It therefore cannot grow in open wounds exposed to the air, and is incapable of development in the blood of a living animal. It differs in another particular, also, viz., in the formation of "spores," which are developed in the rods - one at the end of each bacillus. These are spherical, highly refractive bodies, which resist desiccation, and may retain their vitality for months and probably for years when present in surface soil or in dust. A temperature of $2 \mathrm{I} 2^{\circ} \mathrm{Fahr}$. is required for their destruction.

When a needle is dipped into a pure culture of the tetanus bacillus, and a mouse is inoculated with it, subcutaneously, the animal falls sick within twentyfour hours and dies of typical tetanus in two or three days. The tetanic symptoms are first developed in 
the vicinity of the point of inoculation. In inoculated animals, and in tetanus in man resulting from accidental infection, the bacillus may be obtained in the vicinity of the inoculation wound, but is not present in the blood or in the various organs of the body. The presence of the deadly tetanus toxin may, however, be demonstrated by injecting the blood of a victim of the disease into a mouse, which dies with the characteristic tetanic symptoms after such an inoculation. The fatal dose of this toxin is so small that, according to the Japanese bacteriologist Kitasato, the amount of a culture, from which all living germs have been removed by filtration, which is required to kill a mouse is not more than one hundredthousandth of a cubic centimetre (0.0000 I c.c.). The tetanus poison is destroyed by five minutes' exposure to a temperature of $65^{\circ} \mathrm{C}$. It is also destroyed by exposure to direct sunlight, but it may be kept indefinitely in a cool dark place. The German chemists, Brieger and Cohn, have obtained the toxin in a precipitated and comparatively pure state, in the form of yellowish transparent scales, which are readily soluble in water. They report that this purified toxin will kill a mouse in the dose of 0.00000005 gram, and they estimate that 0.00023 gram would be a lethal dose for a man. Comparing this with the most deadly vegetable alkaloids known, it is nearly six hundred 
times as potent as atropin and one hundred and fifty times as potent as strychnin.

It has long been known that persons who go barefoot are more liable to contract tetanus than those who wear shoes. This is shown by the difference in the mortality between native soldiers and English troops in India. Statistics show that the mortality is higher among males than among fernales. This, of course, depends upon the fact that they are more outof-doors and are more likely to receive accidental wounds. It is a rather remarkable fact that in the United States more deaths occur from tetanus in cities than in the rural districts. This is no doubt largely due to the considerable number of fatal cases of tetanus which occur among boys as a result of lacerated wounds of the hand made by toy pistols, which are so popular as a means of celebrating Independence Day.

The use of these pistols has now been prohibited by several State Legislatures. Dr. Park of Buffalo, who has given special attention to the subject, reports that in Chicago during the month of July, I88 I, sixty cases of tetanus occurred as a result of injuries inflicted by toy pistols. In New York City there were, from the same cause, 38 cases in 1899,33 cases in 1900 , and 27 cases in 1901 .

During our Civil War the total number of cases of 
tetanus reported, as a complication of gunshot wounds, was 505 . This is about one case in every five hundred cases of gunshot injury. The proportion has been much greater in wars conducted by other armies in tropical countries. The total number of deaths from tetanus in the United States during the census year 1900 was 2259 , of whom 1516 were males and 743 females. This includes the deaths from "trismus neonatorum "- tetanus of the new-born. Among the poorer classes in southern localities, and especially among the coloured population, tetanus not infrequently results in the new-born from infection through the navel. In the registration area the highest mortality from tetanus occurred in the States of Vermont and New Jersey. That the mortality reported is chiefly among new-born infants is shown by the fact that of the total number of deaths, I 17.7 per 100,000 were among children under one year of age, while the mortality between the ages of five to fourteen was only 3.7 , and between fifteen and forty-four only 1.7 per 100,000 of these ages. The victims of the toy pistol are, for the most part, included in the group between the ages of five and fourteen. The deaths below the age of one year, constituting a large share of the total number, may justly be classed as victims of dirt. The number of deaths among the new-born was still 
greater in the non-registration area, which includes the Southern States-viz., 234.5 per 100,000 infants in cities. The death-rate among coloured infants was far in excess of that among whites-in the registration area : 77.6 whites, I 233.4 coloured, per 100,000 children under one year of age.

The facts stated indicate the proper preventive measures in this infectious disease: the thorough cleansing of all wounds and especially of penetrating and lacerated wounds and the use of antiseptic dressings by which the multiplication of the tetanus bacillus will be prevented, if by chance it has been introduced; the banishment of the deadly toy pistol; cleanliness and antiseptic dressings of the navels of new-born children; and last, but not least, the education of the public generally as to the manner in which wounds become infected with the tetanus bacillus and the great danger attending such infection. 


\section{CHAPTER XX}

\section{HYDROPHOBIA}

\section{$A$ NOTHER fatal form of wound infection may $A$ result from the bite of a rabid animal - " hydro-}

phobia." The germ of this infectious malady has not been discovered, but it has been demonstrated by experiment that it is present in the saliva of rabid animals and in the nervous tissues - brain and spinal cord - of men and animals who succumb to the disease. A considerable interval elapses after inoculation before the first symptoms of the disease are manifested. This period of incubation varies greatly in its duration, but is rarely less than two weeks or more than six months. By far the largest majority of the cases are developed within three months from the time the bite is inflicted. In the dog the period of incubation usually does not exceed two months. When numerous and severe lacerations have been inflicted, especially if these are upon the face, the disease is apt to develop at a comparatively early 
date. Bites upon the extremities, especially when the teeth of the rabid animal have passed through the clothing, by which the virus is to some extent removed, are less likely to be followed by an attack of hydrophobia.

Among the lower animals the following have been demonstrated to be susceptible to rabies: dogs, cats, cattle, sheep, horses, goats, swine, mice, rabbits, guinea-pigs, skunks.

Man usually contracts the disease through the bites of dogs, cats, or wolves, and occasionally of skunks. A considerable proportion of those who are bitten by rabid animals may escape the disease, especially when the bites are upon the extremities and are not severe. Prompt cauterisation of the wound also has the effect of reducing the proportion of these attacks.

The popular idea that dogs are especially liable to go mad in summer, during "the dog days," appears not to be well founded, as it may prevail at any season, and dogs do not go mad any more than men, unless they have been bitten by a rabid animal and the virus of the disease has been introduced into the wound; or, as has occasionally happened, the infectious material has been introduced into an accidental wound inflicted in some other way.

It has been claimed that certain parts of the world are free from rabies and that it does not prevail in 
Egypt or in the city of Constantinople, which is noted for the number of its homeless dogs. But this is denied by other authorities and it is said to have been very prevalent in the city of Constantinople in 1839 . Like other infectious diseases its prevalence varies greatly at different times and depends to a considerable extent upon the measures taken to prevent its extension - such as the muzzling of dogs and the destruction of those without owners.

In northern Europe rabies from the bite of a mad wolf is of not infrequent occurrence and the disease is also occasionally contracted as a result of bites inflicted by foxes, jackals, and ferrets. Wild animals during the excited stage of the disease lose their fear of man and are liable to run through frontier settlements and military posts, and to bite men and animals encountered on their way.

A most effectual way of resisting the spread of rabies is by the general muzzling of dogs allowed to run at large. The results of this preventive measure as applied in Great Britain are given below. According to official reports the number of fatal cases of rabies in 1887 was 217 ; in 1888,160 ; in $1889,312$. This increase in the number of deaths from hydrophobia caused much alarm and led to the enforcement of regulations for the muzzling of dogs. As a result of this the number of cases fell to 129 in 1890 ; 
79 in 1891 , and 38 in 1892 . There was at this time much opposition to the muzzling ordinance and it was not enforced. As a result of this the number of cases again increased until in the year 1895 it reached highwater mark, 672 cases. Again the muzzling ordinance was enforced, with the result that the number of cases fell to 17 in $1898 ; 9$ in 1899 , and zero in 1900.

The number of deaths from rabies in the United States is not shown in the census reports, the cases being no doubt included under the heading, "Other Causes." In the census of $1900,33,776$ deaths are included under this heading.

It is unfortunate that we have no exact statistics with reference to this disease, inasmuch as its existence has been denied by certain members of the medical profession.

It is difficult to understand the mental operations of those who deny the existence of hydrophobia, the value of vaccination, and other well-established facts, except in the case of those who are ignorant. But for certain minds the logic of facts appears to have no weight as opposed to prejudice and preconceived theories. That there is an infectious disease, known to us as rabies, which is communicated from one animal to others and from rabid animals to man, by the introduction of infectious material contained in the salivary secretions into a wound-usually inflicted by 
the rabid animal's teeth-is as well established as any fact in medicine or in history. But fortunately the disease is rare and comparatively few physicians have been called upon to treat a case of it. It is reported that ninety-one persons have died of hydrophobia in the city of Chicago during the past ten years.

The disease was known to the ancients and is very clearly described by Celus (B. C. 2 I). As long ago as 1813 the French physicians Magendie and Bouchet produced rabies in dogs by inoculating them with saliva obtained from a man suffering from hydrophobia. But our exact knowledge of the disease dates from the researches of the famous French chemist, Pasteur ( 188 I to 1886). Pasteur first announced his success in reproducing rabies in susceptible animals by inoculations with material from the nervous system - brain, spinal cord-in a communication made to the French Academy of Sciences on May 30, r88I. At the same time he reported his success in the discovery of " a method for considerably shortening the period of incubation in rabies and also of reproducing the disease with certainty." This was by inoculations, made after trephining, upon the surface of the brain, with material obtained from the brain of a rabid animal. Dogs inoculated in this way developed rabies in the course of two weeks and died before the end of the third week. In 
a second communication (December I I, I 882) Pasteur reported his success in communicating the disease by intravenous injections of virus obtained from the central nervous system of rabid animals; also the experimental demonstration of the fact that all forms of rabies may be produced by the same virus; also, that all parts of the spinal cord of rabid animals are virulent, as well as all parts of the brain ; also that an animal (dog) which had recovered from a mild attack after inoculation proved to be subsequently immune, and that "this observation constitutes a first step toward a discovery of the prophylaxis of rabies." In a subsequent communication (May 19, I884) Pasteur presented evidence which demonstrated the fact that by successive inoculations in monkeys the period of incubation is prolonged and the virus of the disease attenuated; that this attenuated (milder) virus from the monkey when inoculated into a dog no longer produces fatal rabies; and that dogs so treated are subsequently immune.

Having demonstrated these important facts Pasteur determined to make a test experiment which should convince the scientific world of the truth and value of his discoveries. At his request a commission was appointed by the Minister of Public Instruction to determine the efficacy of his method as applied to the protection of dogs. In his address before the 
International Medical Congress at Copenhagen (August I I, I 884) Pasteur gives the following account of the results of this test experiment.

He says that he gave to the commission nineteen dogs which had been rendered immune against rabies by preventive inoculations. These nineteen dogs and nineteen control animals, obtained from the public pound, without any selection, were tested at the same time. The test was made upon some of the animals of both series by inoculations with virulent material from rabid animals, made upon the surface of the brain, by trephining; and upon others by allowing them to be bitten by rabid dogs; and upon still others by intravenous inoculations. Not one of the protected animals developed rabies; on the other hand, three of the control animals out of six bitten by mad dogs developed the disease; five out of seven which received intravenous inoculations died of rabies; and five which were trephined and inoculated upon the surface of the brain died of the same disease. In a subsequent report the commission, of which $\mathrm{M}$. Bouley was president, stated that twenty-three dogs, which had been protected, were bitten by mad dogs and that all remained in perfect health, while sixty-six per cent. of the control animals, bitten in the same way, developed rabies within two months.

Evidently this method could be applied upon a 
large scale for the prevention of rabies among dogs, and if these animals were thus protected, rabies would soon become practically extinct. The method is even more reliable than vaccination as a protection against smallpox. But its practical application on a large scale would be attended with great difficulties and would, no doubt, be opposed by a large proportion of the owners of dogs. There has, therefore, so far as I am informed, never been any attempt to apply this discovery of Pasteur's in a practical way for the prevention of rabies. But these preliminary experiments led to the discovery that animals and man may be rendered immune to the disease by protective inoculations made after they have been bitten by a rabid animal. I shall not attempt to give an account of the experiments which led Pasteur to this important discovery or of the methods employed for obtaining an attenuated virus, but will content myself with a summary statement of the results which have been attained in the practical application of this method of prevention.

It is probably generally known that " Pasteur Institutes" for the treatment of persons bitten by rabid animals have now been established in all parts of the civilised world. During the year I89 I, I564 persons were inoculated at the Pasteur Institute in Paris with a total mortality of 0.57 per cent. In 324 of 
these cases the animal which inflicted the bite was proved to be rabid by experimental inoculations made in other animals - with an emulsion of the brain or spinal cord. This is now generally recognised as a conclusive demonstration that an animal, or man, from whom such virulent material has been obtained, was a victim of rabies.

Perdrix ( 1890$)$ in an analysis of the results obtained at the Pasteur Institute in Paris calls attention to the fact that the mortality among those treated has diminished each year and ascribes this to improvements in the method employed. In the cases with severe wounds upon the head and face larger doses of the virulent material are used at more frequent intervals. Perdrix gives the following statistics with reference to the location of the bite as influencing the results of treatment.

Bitten upon the head, 684 ; died, $12=1.75 \%$
Bitten upon the hands, $4396 ;$ died, $9=0.2 \%$
Bitten upon the limbs, $2839 ;$ died, $5=0.17 \%$

Recently the statistics of the Pasteur Institute in Paris for a period of sixteen years (I886 to I90I) have been published. During this period I 12 deaths occurred among 25,986 persons inoculated-a mortality of $0.43 \%$. It is not claimed that all of the inoculated had been bitten by rabid animals. In many cases it is impossible to ascertain whether the 
animal which inflicted the bite was really mad. But the cases in which satisfactory experimental proof has been obtained are considered separately in the statistics of the Pasteur Institute. Thus, in the year I90I, I 7I persons were treated who had been bitten by animals proved to be rabid, and among these not a single death occurred. 


\section{INDEX}

Acquired immunity, 73

Alcohol as an antiseptic, 4I

Amœbic dysentery, 140

Anopheles, 235

Anthrax, 23

Antiseptic, definition of, 29

- surgery, 269

Antiseptics, 4I

Antitoxin of diphtheria, 198

Antitoxins, 82

Aseptic surgery, 269

Asiatic cholera, I Io

Attenuation of virulence, 64,78

Bacilli, Io

Bacillus of bubonic plague, IOI

- diphtheria, 193

- influenza, 200

- leprosy, 182

- tetanus, 273

- tuberculosis, I60

- typhoid fever, 136

Bacteria, 37

- multiplication of, $\mathbf{I I}$

- structure of, 13

- spores of, 12

Bichloride of mercury, 6r

Black death, 9I, 93

Bleaching powder, 60

Blood-poisoning, 20

- germs of, 265-269

Blood serum, germicidal action of, 68,268
Breathing exercises, 176

Bubonic plague, 89

- bacillus of, ror

- in Philippine Islands, IO2

- mortality from, 94-98

- period of incubation, 105

- prevention of, 108

- protective inoculations, I07

Carbolic acid as a disinfectant, 59

Caustic alkalies as disinfectants, 58

Channels of infection, 14

Chicken-pox, 22I

Children, susceptibility of, 25

Chloride of lime, 60

Chlorin as a germicide, 56 ,

Chlorinated lime, 60

Cholera, I Io

- germs of, I20

- in America, III-II6

- origin of epidemics, 117,118

- predisposing causes, 118

- protective inoculations, 124

- recent epidemics, 116

Cholera germ, description of, 120

- destruction of, I1 8, 122

- discovery of, 120

- infection by, II 8 , II 9

Cholera infantum, I40

Citric acid as a germicide, 57

Climate in treatment of consumption,

177

Clothing, disinfection of, 47 
Clothing, infection through, 18

Coal-tar products as germicides, 59

Cold, action of, on bacteria, 46

Colonies of bacteria, I I

Comma bacillus, $\mathbf{2} 20$

Contagion, definition of, 3

Corrosive sublimate as a disinfectant, 61

Creolin as a disinfectant, 60

Cresol as a disinfectant, 60

Culture experiments, 37

- fluids, 38

Cuspidors for consumptive patients, I71, 172

Deodorants, 30

Desiccation of bacteria, 46

Diphtheria, I9I

- animals susceptible to, 192

- antitoxin of, 198

- bacillus of, 193

- disinfection in, 195

- mortality from, 194

- prevention of, 195

- propagation of, 191

Diphtheria bacillus, 193

- destruction of, 195

- discovery of, 192

- varieties of, 192

Disease germs, 8

- dates of discovery, $\mathbf{1 4 8}$

Disinfectant, definition of, 28

Disinfection, 28

- by gases, 5 I

- evidence of, 34

- in bubonic plague, 108

- in cholera, 122, 123

- of bed-linen, 60

- of clothing, 47

- of excreta, 47,60

- of sick-room, 5I-53, I 74

- of sputa, 171

- tests of, 32
Dry heat as a disinfectant, 44

Dust, infection through, I6, 5 I

Dysentery, 140

- mortality from, I4I.

Ehrlich's side chain theory, 85

Electric light, germicidal action of, 49

Epidemics, termination of, 99

Eruptive fevers, 17

Erysipelas, germ of, 266, 267

Excreta, disinfection of, 47,60

Famine fever, $15 \mathrm{I}$

Filth diseases, 103, 126

Flies, as carriers of infection, 18

Fomites, 3

Formaldehyd as a disinfectant, 54

Fumigation, 53

German measles, 233

Glanders, 24

Heat, disinfection by, 43

Hospital gangrene, 19

Hydrophobia, 279

- mortality from, 282

- Pasteur's experiments, 283

- period of incubation, 279

- prevention of, 28I

- protective inoculations, $283-288$

- susceptible animals, 280

- transmission of, 284

Immunity, acquired, 73

- due to alkalinity of blood, 67

- due to body temperature, 66

- natural, 63

Infection, definition of, 4,7

- general remarks upon, 3

Influenza, 199

- bacillus of, 200

- bacillus, destruction of, 204

- complications of, 200

- epidemics of, 200-202 
Influenza, incubation period, 202

- mortality from, 199. 200

- predisposing causes, 202

- prevention of, 203

Inoculation for smallpox, 76

Lepers, isolation of, 189

Leprosy, I8I

- bacillus of, 182

- disinfection in, Igo

- history of, 186

- in Great Britain, 187

- in India and China, 187

— in Norway, 188

- in United States, 189

- inoculation experiments, 182

- transmission of, 186

Leucocytes, phagocytic action of, 7 I

Lime as a disinfectant, 59

Lye as a disinfectant, 58

Lysol, 60

Malaria, 235

Malarial fevers, 234

- fevers, geographic distribution of, 245

- fevers, mortality from, 247

- fevers, period of incubation. 240

- fevers, prevention of, 249-25 I

- fevers, seasonal prevalence of, 246

- parasite, 237, 24I

- parasite, discovery of, 234

- parasite, varieties of, 240-242

Measles, 228

- complications of, 230

- disinfection in, 230, 231

- mortality from, 229

- period of incubation, 228

- prevention of, 23I

- second attacks, 233

Membranous croup, 194

Metchnikoff's theory, 72

Micrococci, I6
Micrococcus of erysipelas, 265, 267

- of pneumonia, 206

- of pneumonia, discovery of, 205

- of pneumonia, where found, 208

- of puerperal fever, 266

Micro-organisms, 8

Milk, antitoxins in, 82

- from tuberculous cows, 175

Mineral acids, as germicides, 57

Mortality of armies, I29, 143

Mosquitoes, infection by, $2 \mathrm{I}$

- malarial parasite in, 243

- transmission of yellow fever by, 255

Mucous membrane, infection through, 21

Natural immunity, 63

Oysters, infection by, 18

Pasteur Institute, statistics of, 287 , 288

Period of incubation, 6

Phagocytosis, 71, 72

Plasmodium malariæ, 237

Pneumonia, 205

- epidemics of, 209

- in Chicago, 207

- in United States, 206

- mortality from, 207

- predisposing causes, 206

- prevention of, 210

Pocket flasks for consumptives, 172

Potash as a germicide, 58

Protective inoculations, $75-78$

- inoculations in bubonic plague, 107, 108

- inoculations in cholera, 124

- inoculations in typhoid fever, 139

Protophyta, 10

Protozoa, 10

Ptomaines, 30 
Puerperal fever, germ of, 266, 267 Pulmonary consumption, mortality from, $160-162$

Pure cultures, 38

Race susceptibility, 24

Rats as agents in spread of plague, I04

Relapsing fever, 145

- epidemics of, 146,147

- germ of, $\mathbf{1 4 8}$

- in United States, I47

- predisposing causes, $15 \mathrm{I}$

- prevention of, 152

Salt as an antiseptic, $4 \mathbf{I}$

Sanitoria in treatment of tuberculosis, 169

Saprophytes, 86, 267

Scarlet fever, 223

- history of, 223

- how communicated, 223

- mortality from, 225

- prevention of, 226

Second attacks of infectious diseases, 73-74

Septicæmia, 19

Sewers, sanitary value of, 127

Ship fever, 157

Sick-room, disinfection of, $5 \mathrm{I}$

Skin, infection through, 2 I

Smallpox, 214

- disinfection in, 220

- duration of, 220

- germ of, 219

- history of, 214

- mortality from, 215

- second attacks of, 74

Snake poison, antitoxin of, 85

Soda as a germicide, 59

Spirilla, ro

Spirillum of cholera, 121

- of relapsing fever, 149

Spores, 12, 39
- of tetanus bacillus, 273, 274

- resistance to heat, 44

Sputum, disinfection of, I $7 \mathrm{I}$

Staphylococcus pyogenes aureus, 265

Steam as a disinfectant, 44

Streptococcus pyogenes, 267

Summer complaint, 140

Sulphate of copper as a germicide, 62

- iron as a germicide, 29

Sulphur fumigation, 53, 54

Sulphurous acid as a germicide, 58

Sunlight as a disinfectant, 48

Susceptibility, individual, 25

- due to alcoholism, 68

- race, 23

Surra disease, 22

Tetanus, 272

- bacillus of, 273

- mortality from, $276-278$

- toxin, 273-275

Texas fever, $2 \mathrm{I}$

Thermal death-point of bacteria, 45

Ticks, infection by, 21

Tonsils, infection through, I5

Toy pistols, danger of, 276

Trichinosis, 19

Trismus neonatorum, 277

Tsetse-fly disease, 22

Tubercle bacillus, 160

- destruction of, $\mathbf{I} 76$

- discovery of, I59

- in milk, 175

Tubercular joint disease, 163

Tuberculosis, 159

- communication of, 159-163

- in Europe, 166

- in New York City, 161, 178

- influence of occupation, 165

- - race, 166

- mortality from, I60, I62

- of bowels, 163

- predisposing causes of, 164 
Tuberculosis, prevention of, 170

- susceptibility to, 164

- treatment of, 167

Typhoid bacillus, discovery of, 136

- description of, $\mathrm{t}_{3} 6$

- destruction of, 138

- in urine, 135

Typhoid fever, $\mathbf{I} 26$

- bacillus of, 138

- mortality from, I26-134

- preventive inoculations, I39

- second attacks of, 74

Typhus fever, 154

- causes of, 157

- epidemics of, $154-156$

- in United States, 156

- mortality from, 158

Uncinaria Americana, I42 United States Army, mortality in, I43 Urine, typhoid bacilli in, I 35
Vaccination, 76, 221

- discovery of, 218

- value of, $216-218$

Vaccine virus, 36

Violet light, germicidal action of, 49

Water-born diseases, I5

Water supply, infection of, 133

Whitewash as a disinfectant, 59

Whooping-cough, 2 II

- mortality from, 212

- prevention of, 213

Wound infections, I9, 264

Yellow fever, 252

- board to investigate, 255

- epidemics in United States, 253254

- geographic range, 252-254

- prevention of, $25^{8-265}$

- transmission of, 255 



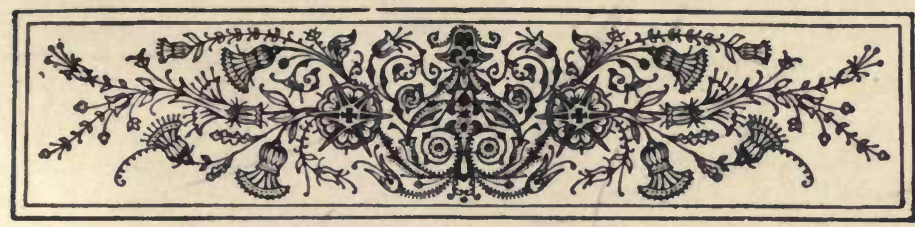

\section{The Science Series}

Edited by Professor J. McKeen Cattell, Columbia University, with the coöperation of FranK Evers BEDI)ARD, F.R.S., in Great Britain.

Each volume of the series will treat some department of science with reference to the most recent advances, and will be contributed by an author of acknowledged authority. Every effort will be made to maintain the standard set by the first volumes, until the series shall represent the more important aspects of contemporary science. The advance of science has been so rapid, and its place in modern life has become so dominant, that it is needful to revise continually the statement of its results, and to put these in a form that is intelligible and attractive. The man of science can himself be a specialist in one department only, yet it is necessary for him to keep abreast of scientific progress in many directions. The results of modern science are of use in nearly every profession and calling, and are an essential part of modern education and culture. A series of scientific books, such as has been planned, should be assured of a wide circulation, and should contribute greatly to the advance and diffusion oi scientific knowledge.

The volumes will be in octavo form, and will be fully illustrated in so far as the subject-matter calls for illustrations. 


\title{
THE SCIENCE SERIES
}

\author{
Edited by J. McKeen Cattell, M.A., Ph.D., and F. E.
} BEDUARD, M.A., F.R.S.

I.-The Study of Man. By Professor A. C. HADdon, M.A., D.Sc., M.R.I.A. Fully illustrated. $8^{\circ}, \$ 2.00$.

"A timely and useful volume. . . The author wields a pleasing pen and knows bow to make the subject attractive. . . The work is calculated to spread among its readers an attraction to the science of anthropology. The author's observations are exceedingly genuine and his descriptions are vivid."-London Atkenaum.

2.-The Groundwork of Science. A Study of Epistemology. By St. George Mivart, F.R.S. \$1.75.

"The book is cleverly written and is one of the best works of its kind ever put before the public. It will be interesting to all readers, and especially to those interested in the study of science."-New Haven Leader.

3.-Rivers of North America. A Reading Lesson for Students of Geography and Geology. By Israfel C. Russell, Professor of Geology, University of Michigan, author of "Lakes of North America," "Glaciers of North America," "Volcanoes of North America," etc. Fully illustrated. $8^{\circ}, \$ 2.00$.

"There has not been in the last few years until the present book any authoritative, broad résumé on the subject, modified and deepened as it has been by modern research and reflection, which is couched in language suitable for the multitude. . . . The text is as entertaining as it is instructive."-Boston.Transcript.

4.-Earth Sculpture; or, The Origin of Land-Forms. By James GeIKIE, LL.D., D.C.L., F.R.S., etc., Murchison Professor of Geology and Mineralogy in the University of Edinburgh; author of "The Great Ice Age," etc. Fully illustrated. $8^{\circ}, \$ 2.00$.

"This volume is the best popular and yet scientific treatment we know of of the origin and development of land-forms, and we immediately adopted it as the best available text-book for a college course in physiography. . . . The book is full of life and vigor, and shows the sympathetic touch of a man deeply in love with nature."-Science.

5.-Volcanoes. By T. G. Bonney, F.R.S., University College, London. Fully illustrated. $8^{\circ}, \$ 2.00$.

"It is not only a fine piece of work from a scientific point of view, but it is uncommonly attractive to the general reader, and is likely to have a larger sale than most books of its class." -Springfield Republican.

6.-Bacteria : Especially as they are related to the economy of nature, to industrial processes, and to the public bealth. By GeORGe Newman, M.D., F.R.S. (Edin.), D.P.H. (Camb.), etc., Demonstrator of Bacteriology in King's College, London. With 24 micro-photographs of actual organisms and over 70 other illustrations. $8^{\circ}, \$ 2.00$.

"Dr. Newman's discussions of bacteria and disease, of immunity, of antitoxins, and of methods of disinfection, are illuminating, and are to be commended to all seeking information on these points. Any discussion of bacteria will seem technical to the uninitiated, but all such will find in this book popular treatment and scientific accuracy happily combined."-The Dial. 
7.-A Book of Whales. By F. E. BEDDARD, M.A., F.R.S. Illustrated. $8^{\circ}, \$ 2.00$.

"Mr. Beddard has done well to devote a whole volume to whales. They are worthy of the biographer who has now well grouped and described these creatures. The general reader will not find the volume too technical, nor has the autbor failed in his attempt to produce a book that shall be acceptable to the zoölogist and the naturalist." $-N$. Y. Times.

8.-Comparative Physiology of the Brain and Comparative Psychology. With special reference to the Invertebrates. By JACQUES LoEB, M.D., Professor of Physiology in the University of Chicago. Illustrated. $8^{\circ}, \$ 1.75$.

"No student of this most interesting phase of the problems of life can afford to remain in ignorance of the wide range of facts and the suggestive series of interpretations which Professor Loeb has brought together in this volume."-JosePH JASTROw, in the Chicago Dial.

9. - The Stars. By Professor Simon Newcomb, U.S.N., Nautical Almanac Office, and Johns Hopkins University. $8^{\circ}$. Illustrated. Net, \$2.00. (By mail, \$2.20.)

ro.- The Basis of Social Relations. A Study in Ethnic Psychology. By Daniel G. Brinton, A.M., M.D., LL.D., Sc.D., Late Professor of American Archæology and Linguistics in the University of Pennsylvania; Author of "History of Primitive Religions," "Races and Peoples," "The American Race," etc. Edited by Livingston FarRAND, Columbia University. $8^{\circ}$. (By mail, \$1.60.) Net, \$r.50.

II.-Experiments on Animals. By Stephen PAfET. With an Introduction by Lord Lister. Illustrated. $8^{\circ}$, net, \$2.00. (By mail, \$2.20.)

12.-Infection and Immunity. With Special Reference to the Prevention of Infectious Diseases. By George M. Sternberg, M.D., LL.D., Surgeon-General U. S. Army (Retired). Illustrated. $8^{\circ}$.

\section{The following volumes are in preparation :}

Meteors and Comets. By Professor C. A. Young, Princeton University. The Measurement of the Earth. By Professor C. T. Mendenhall, Worcester Polytechnic Institute, formerly Superintendent of the U. S. Coast and Geodetic Survey.

Earthquakes. By Major C. E. Dutron, U.S.A.

The History of Science. By C. S. Peirce.

Recent Theories of Evolution. By J. Mark Baldwin, Princeton University.

The Reproduction of Living Beings. By Professor Marcus Hartog, Queen's College, Cork.

Man and the Higher Apes. By Dr. A. KeIth, F.R.C.S.

Heredity. By J. Arthur Thompson, School of Medicine, Edinburgh.

Life Areas of North America: A Study in the Distribution of Animals and Plants. By. Dr. C. Hart Merriam, Chief of the Biological Survey, U. S. Department of Agriculture.

Age, Growth, Sex, and Death. By Professor Charles S. Minot, Harvard Medical School.

History of Botany. By Professor A. H. Green.

Planetary Motion. By G. W. Hill.

Infection and Immunity. By Georee M. Sternberg, Surgeon-Gereral, U.S.A. 





$$
\text { YCl10613 }
$$




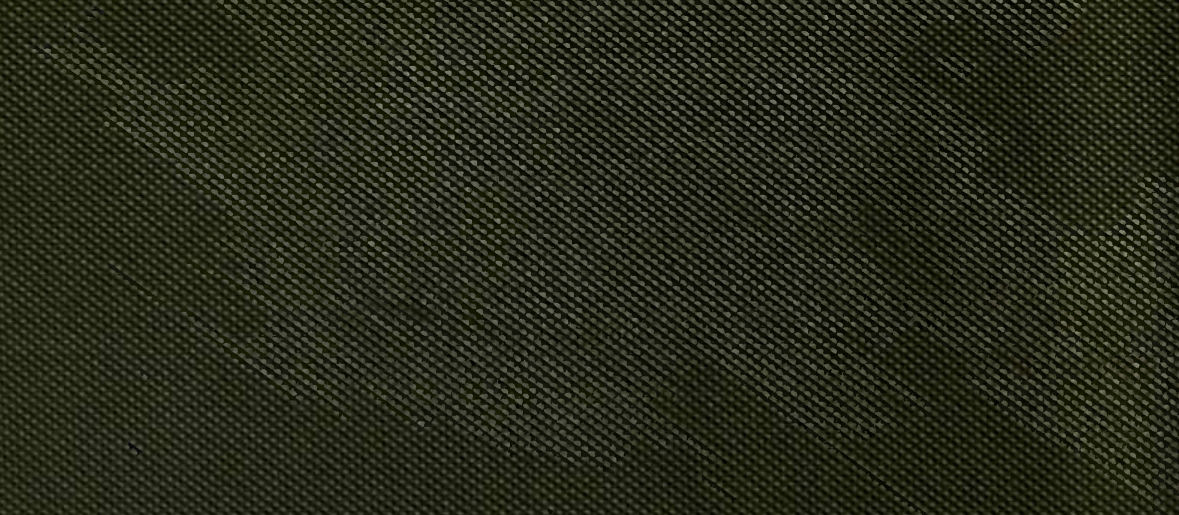

